\title{
Ex-post Evaluation des Forschungsprogramms „Forschung für die Produktion von morgen“" für die Jahre 1999 bis 2004
}

Endbericht

Anton Geyer (Projektleiter)

Joachim Hafkesbrink

Steffen Kinkel

14. November 2006

Technopolis

Forschungs- und Beratungsgesellschaft $\mathrm{mbH}$

Prinz Eugen Straße 80/12

1040 Wien

Fraunhofer Institut

Tel. +43 (1) 5039592 - 16

System- und Innovationsforschung

anton.geyer@technopolis-group.com

www.technopolis-group.com 


\section{Ex-post Evaluation des Forschungsprogramms „Forschung für die Produktion von morgen“ für die Jahre 1999 bis 2004}

Endbericht

Anton Geyer (Projektleiter)

Joachim Hafkesbrink

Steffen Kinkel

Evaluationsteam:

ARÖW GmbH, Duisburg

Joachim Hafkesbrink, Michael Krause, Monika Rudeloff, Markus Schroll

Fraunhofer-Institut für System- und Innovationsforschung, Karlsruhe Barbara Breitschopf, Bernd Ebersberger, Angela Jäger, Steffen Kinkel, Spomenka Maloca, Oliver Som

Technopolis GmbH, Wien Anton Geyer, Alexandra Rammer, Jürgen Streicher

Internationales Experten Panel

Prof. John Bessant (Imperial College London) Prof. Claudio Boër (Swiss Innovation Promotion Association) Prof. Harry Boer (Universität Aalborg) Jan Willem Gunnink (TNO Eindhoven)

Jos Pinte (Agoria Brüssel)

Prof. Reijo Tuokko (Universität Tampere)

Prof. Konrad Wegener (ETH Zürich) 


\section{Vorbemerkung}

Das Bundesministerium für Bildung und Forschung, Referat 512 Produktionssysteme und -technologien, beauftragte im Juni 2005 die Technopolis Forschungsund Beratungsgesellschaft mbH, Wien, in Zusammenarbeit mit dem FraunhoferInstitut für System- und Innovationsforschung (FhG-ISI), Karlsruhe, und der ARÖW Gesellschaft für Arbeits-, Reorganisations- und ökologische Wirtschaftsberatung $\mathrm{mbH}$, Duisburg mit der ex-post Evaluation des BMBF Programms „Forschung für die Produktion von morgen" für die Jahre 1999 bis 2004.

Ziel der Evaluation war es, die Wirkungen der Programmförderung im Bereich Produktionsforschung vor dem Hintergrund der im BMBF Rahmenkonzept ,Forschung für die Produktion von morgen' formulierten Ziele zu erheben und zu bewerten. Hierbei waren die Art der Programmdurchführung und die Möglichkeiten und Grenzen des Förderinstruments Verbundforschung angemessen zu berücksichtigen. Darüber hinaus sollte die Evaluation eine Standortbestimmung über Produktion und Produktionsforschung in Deutschland seit Beginn des aktuellen BMBF Rahmenkonzepts ermöglichen. Dazu liefert die Evaluation Informationen und Kennziffern über die Struktur und die Entwicklung der produktionstechnischen Wirtschaft und Wissenschaft in Deutschland seit dem Jahr 1999.

Im Zentrum der Evaluation stand aber die Beschreibung des ökonomischen und gesellschaftlichen Nutzens, der mit den eingesetzten Ressourcen bisher erzielt werden konnte. Hierbei wurden als Indikatoren unter anderem der Einfluss des Programms auf die Forschungsausgaben der beteiligten Unternehmen sowie auf deren technologische und organisatorische Kompetenz herangezogen. Auch die direkte Verwertung der Ergebnisse durch neue Produkte, Prozesse und produktbegleitende Dienstleistungen wurde erhoben. Dabei wurde auch ein Vergleich mit strukturell ähnlichen, nicht geförderten Unternehmen durchgeführt. Besonderes Augenmerk legte das Evaluationsteam auf die Frage, in welchem Ausmaß kleine und mittlere Unternehmen (KMU) mit dem Programm erreicht werden konnten und von der Teilnahme an den Verbundprojekten profitiert haben. Außerdem wurde das Zusammenwirken von Entwicklern, Zulieferern, Dienstleistern und Anwendern in den geförderten Verbünden genauer analysiert.

Schließlich wurde die operative Programmumsetzung durchleuchtet. Das Zusammenwirken von BMBF, dem Projektträger PTKA-PFT in Karlsruhe und den Beteiligten aus Industrie, Forschung und Verbänden bei der Durchführung und Weiterentwicklung des Programms war ebenso Thema der Evaluation wie die Antragsbearbeitung, die Projektbetreuung, Transfermaßnahmen und die Öffentlichkeitsarbeit im Programm.

Die Informationen und Analysen in dieser Evaluation bieten dem BMBF eine solide empirische Grundlage zur Weiterentwicklung seiner Fördermaßnahmen im Lichte der gemachten Erfahrungen und Ergebnisse in der Programmperiode 1999 bis 2004. Bei der Erarbeitung seiner Empfehlungen stützte sich das Evaluationsteam auch auf die Diskussionen eines Experten Panels, dem sieben internationale Experten aus den Bereichen Produktionsforschung und Innovationsforschung angehörten. Die vom Evaluationsteam vorgeschlagenen konkreten Handlungsempfehlungen zur Weiterführung von „Forschung für die Produktion von morgen“ können dem hier vorliegenden zusammenfassenden Endbericht entnommen werden. 


\section{Kurzfassung}

Seit Ende 1999 fördert das BMBF mit dem Rahmenkonzept „Forschung für die Produktion von morgen" Forschung in Verbundprojekten mit dem Ziel, beispielhafte Lösungen für die zukunftsfähige Produktion in Deutschland zu entwickeln und Forschungsergebnisse für die breite Anwendung besonders für kleinere und mittlere Unternehmen (KMU) bereitzustellen. Die Evaluation des Rahmenkonzepts „Forschung für die Produktion von morgen“ sollte die Frage beantworten, welchen Beitrag dieses Programm bzw. sein zentrales Instrument der Verbundprojektförderung zur Stärkung der technologischen und wirtschaftlichen Wettbewerbsfähigkeit des deutschen Produktionssektors, insbesondere auch der kleinen und mittleren Unternehmen (KMU), leistet. Die Ergebnisse der Evaluation sollen Hinweise dazu geben, wo im Lichte der Programmzielsetzungen gegebenenfalls die neuralgischen Punkte liegen, an denen Verbesserungen des Programms in Zukunft sinnvoll ansetzen können.

\section{Evaluationsmethodik}

Methodisch wurde in der Evaluation auf einen breiten Mix von quantitativen und qualitativen sozial- und wirtschaftswissenschaftlichen Erhebungs- und Analysemethoden gesetzt (Dokumentenanalyse, Interviews, Fallstudien, statistische Datenauswertung, Teilnehmerbefragung). Durch einen Rückgriff auf die Ergebnisse der FhG-ISI Befragungen ,Innovationen in der Produktion' der Jahre 2003 und 2006 konnte erstmals auch eine Matched-Pair Analyse zu Unterschieden in der Innovationsleistung von geförderten und nicht geförderten Betrieben als Effekte des Rahmenkonzepts durchgeführt werden.

\section{Lässt sich in den Betrieben eine Wirkung des Rahmenkonzepts feststellen?}

Mit der Förderung der Verbundprojekte im Rahmenkonzept wird ein signifikanter Beitrag zur Stärkung der Innovations- und Wettbewerbsfähigkeit der teilnehmenden Unternehmen, besonders auch der KMU, geleistet. Die Ergebnisse der Evaluation zeigen eindeutig signifikante positive Wirkungen des Rahmenkonzepts in den teilnehmenden Betrieben an.

Wie die Ergebnisse der Matched-Pair Analyse zeigen, investieren geförderte Unternehmen mit im Mittel 8,1 Mio. EUR einen deutlich höheren Betrag in FuE als dies die nicht geförderten Zwillinge mit im Durchschnitt 3,7 Mio. EUR tun. Die im jeweiligen Jahr erhaltene Fördersumme wurde von den FuE-Aufwendungen bereits abgezogen, um reine Additionalitäten ausweisen zu können. Entsprechend ist auch die FuE-Intensität der geförderten Betriebe nach Erhalt der Förderung mit $9 \%$ signifikant höher als bei ihren nicht geförderten Pendants, die etwa $7 \%$ FuE-Anteil am Umsatz aufweisen. Ähnliche Befunde zeigen sich für das FuE-Personal als Indikator für die intern erbrachte FuE: Auch hier weisen geförderte Unternehmen mit im Mittel etwa 88 Beschäftigten im Bereich FuE einen deutlich höheren Wert aus als die nicht geförderten Vergleichsunternehmen (49 Beschäftigte) und haben dementsprechend einen signifikant höheren Anteil des FuE-Personals am Gesamtpersonal (16\% gegenüber $13 \%$ ). Die statistisch signifikanten Additionalitätseffekte zeigen sich nicht nur für alle geförderten Unternehmen, sondern auch für die strategisch wichtige Teilgruppe der KMU. Insgesamt führt die Förderung im 
Rahmenkonzept in den geförderten Unternehmen zu einer deutlichen Steigerung der FuE-Anstrengungen über die gewährte Förderung hinaus.

Von den geförderten Betrieben, die ihr FuE-Vorhaben bereits abgeschlossen haben, macht heute bereits ein Viertel (25\%) Umsätze mit Produkten, die unmittelbar auf den Ergebnissen des Projekts aufbauen. 15\% der geförderten Betrieben, die ihr FuEVorhaben bereits abgeschlossen haben, machen heute bereits Umsätze mit Dienstleistungen, die unmittelbar auf den Ergebnissen der FuE-Vorhaben aufbauen. Insgesamt geben fast ein Drittel (32\%) der Betriebe, die ihr FuE-Vorhaben bereits abgeschlossen haben, an, bereits Umsätze mit Produkten und Dienstleistungen zu machen, die auf den Vorhabensergebnissen aufbauen.

In die gleiche Richtung zeigen die Ergebnisse der Matched-Pair-Analyse: Jeweils etwa $10 \%$-Punkte mehr geförderte Unternehmen, die im Vergleich zu ihren ungeförderten Pendants Produkt- oder Marktinnovatoren sind, sprechen eine deutliche Sprache. Diese Ergebnisse sind sowohl für alle geförderten Unternehmen statistisch signifikant wie auch für die Teilgruppe der kleinen und mittleren Unternehmen (KMU), die als besondere Zielgruppe des Rahmenkonzepts gelten.

Insgesamt 15\% der Betriebe, die ihr FuE-Vorhaben bereits abgeschlossen haben, gaben an, dass die Projektergebnisse unmittelbar zu Kostensenkungen im Betrieb geführt haben und 28\% der Betriebe, die ihr FuE-Vorhaben bereits abgeschlossen haben, gaben an, dass sich durch die Verwertung der Projektergebnisse die Ertragsstärke des Betriebs verbessert hätte. Insgesamt konnten bereits $42 \%$ der Betriebe, die ihr FuE-Vorhaben abgeschlossen haben, die Projektergebnisse in dem Sinne verwerten, dass sie entweder Umsätze mit Produkten, Dienstleistungen und Lizenzen verzeichnen konnten oder dass die Projektteilnahme unmittelbar zu geringeren Kosten im Betrieb bzw. zu mehr Ertragsstärke des Unternehmens geführt hat. Angesichts eines vorwettbewerblich orientierten Programms und der vergleichsweise kurzen Zeit, die den Betrieben seit Projektabschluss zur Verfügung stand, die Ergebnisse zu verwerten, sind diese Ergebnisse beachtlich und erfreulich.

Die empirischen Ergebnisse der Teilnehmerbefragung lassen den Schluss zu, dass KMU von der Teilnahme am Programm besonders profitieren, und die erzielten Ergebnisse zur Verbesserung ihrer Kompetenzen am Markt verwerten. Mehr als drei Viertel der KMU kooperieren nach Abschluss der FuE-Vorhaben weiterhin mit ihren industriellen Partnern aus dem Verbund, wobei etwa die Hälfte der KMU dies auch in Form von FuE Kooperationen zu anderen Themen tut. Dies ist ein deutlicher Beleg dafür, dass es dem Rahmenkonzept gelingt, die Zusammenarbeit von KMU in Unternehmensnetzwerken erfolgreich zu unterstützen.

\section{Hat das Rahmenkonzept seine Zielgruppen erreicht?}

Insgesamt wurden mit dem Rahmenkonzept im Evaluationszeitraum 166 Verbundprojekte mit 1.157 geförderten Projektpartnern unterstützt und dafür 264 Mio. EUR Fördermittel aufgewendet. 60\% der geförderten Industriebetriebe sind kleine und mittlere Unternehmen (KMU) mit weniger als 250 Beschäftigten. Von den $63 \%$ der Fördermittel des Rahmenkonzepts, die Industriebetrieben zugute kommen, entfallen $64 \%$ auf KMU. Die realisierte Verteilung kann vor dem Hintergrund der Ziels, 
kleine und mittlere Unternehmen angemessen zu beteiligen, als durchaus gelungen bezeichnet werden.

Die Beteiligungsquote von Betrieben aus den neuen Bundesländern ist mit etwa $10 \%$ etwas geringer als ihr Gesamtanteil am Verarbeitenden Gewerbe (15\%). In diesem Kontext gilt es jedoch zu beachten, dass sich das Rahmenkonzept insbesondere an Betriebe richtet, die eigene FuE betreiben. Verglichen mit dem Anteil der internen betrieblichen FuE-Aufwendungen des Verarbeitenden Gewerbes, der auf Betriebe aus den neuen Bundesländern entfällt (4,2 \%), kann nicht auf eine unterdurchschnittliche Beteiligung ostdeutscher Betriebe geschlossen werden.

Mit über einem Viertel stellt der Maschinenbau die meisten der in den Verbundvorhaben beteiligten Betriebe, wobei insbesondere Hersteller von Werkzeugmaschinen im Vergleich zur Betriebsanzahl im Verarbeitenden Gewerbe überproportional vertreten sind. An zweiter und dritter Stelle der beteiligten Branchen folgen mit knapp $17 \%$ die Hersteller von Geräten der Medizin-, Mess-, Steuerungs-, Regelungstechnik und Optik, von denen insbesondere die Messgerätehersteller überdurchschnittlich partizipieren sowie der Fahrzeugbau, der mit knapp $9 \%$ der beteiligten Betriebe im Vergleich zur Grundgesamtheit ebenfalls überrepräsentiert ist. Mit $18 \%$ überraschend hoch ist die Quote der beteiligten Betriebe aus wissensintensiven produktionsnahen Dienstleistungsbereichen (Datenverarbeitung und Datenbanken, Forschung und Entwicklung, Dienstleistungen überwiegend für Unternehmen). Dagegen sind Betriebe der Prozessindustrie (insbesondere der Chemischen Industrie) sowie Betriebe aus so genannten Low-Tech-Sektoren (Textil, Bekleidung, Leder, Holz, Nahrungsmittel, etc.) signifikant unterdurchschnittlich in den Verbundvorhaben des Rahmenkonzepts vertreten. Damit setzt das Rahmenprogramm schwerpunktmäßig auf die Förderung neuer Lösungen für Stückgut fertigende Betriebe aus dem Bereich der höherwertigen Technologie, die in Deutschland traditionell stark ist und wesentlich zur positiven Handelsbilanz der deutschen Wirtschaft beiträgt.

Industrielle Anwender sind in $46 \%$ der Verbundvorhaben als geförderte Partner integriert. Bezieht man Industriebetriebe mit der Rolle des Entwicklers für die eigene Anwendung mit ein, so kann davon ausgegangen werden, dass in $72 \%$ der Verbundvorhaben zumindest ein Industriebetrieb beteiligt ist, der die Nachfrage- bzw. Anwenderseite vertritt. In diesem Kontext wäre auch zu untersuchen, ob assoziierte Pilotanwender in ungeförderten Arbeitskreisen eine wirksame Alternative zur geförderten Einbindung sein können. Geförderte Transferpartner finden sich in etwa $6 \%$ der Verbundvorhaben, in geschätzten weiteren $6 \%$ wirken assoziierte oder Transferpartner im Unterauftrag mit. Diese vergleichsweise geringe Integrationsquote ist vor dem Hintergrund des Ziels des Rahmenkonzepts, die breite Anwendung der entwickelten Lösung, insbesondere in KMU, sicher zu stellen, vertieft zu beleuchten.

Die überwiegende Mehrzahl der beteiligten Forschungsinstitute stammt aus den Fachbereichen Maschinenbau und Verfahrenstechnik (insgesamt $60 \%$ ). Auf den weiteren Rängen folgen Institute der Elektrotechnik (12\%), der Wirtschaftswissenschaften (10\%), der Informatik (6\%) und der Sozialwissenschaften (4\%). Spezifische technologische Kompetenzen jenseits des traditionellen Maschinenbaus werden insbesondere aus den Bereichen Werkstofftechnologien, Lasertechnik, 
Mikrotechnik bzw. -elektronik sowie mit Abstrichen Oberflächen- oder Beschichtungstechnik mit in die Verbundvorhaben integriert.

\section{Wurde das Rahmenkonzept thematisch angemessen umgesetzt?}

In den zwölf Bekanntmachungen im Evaluationszeitraum 1999 bis 2004 wurden 22 spezifische Themenfelder adressiert. Der Schwerpunkt lag dabei bei den technisch orientierten Themen des Handlungsfelds 2: Technologien und Produktionsausrüstungen. Mehr als die Hälfte der geförderten Verbundprojekte $(55 \%)$ und mehr als zwei Drittel der vergebenen Fördermittel entfallen auf dieses Handlungsfeld. Insgesamt $18 \%$ der Fördermittel wurden für die 38 Verbundprojekte $(23 \%)$ des Handlungsfelds 1: Marktorientierung und strategische Produktplanung gewidmet. 8\% der Fördermittel entfallen auf die 15 Verbundvorhaben des Handlungsfelds 3: Neue Formen der Zusammenarbeit produzierender Unternehmen und nur etwa 5\% der Förderungen im Rahmenkonzept wurden für Vorhaben des Handlungsfelds 4: Der Mensch und das wandlungsfähige Unternehmen eingesetzt. Vor dem Hintergrund des systemischen Anspruches des Rahmenkonzepts „Forschung für die Produktion von morgen" und seiner spezifischen Zielsetzungen stellt sich die Frage, ob eine stärkere Berücksichtigung der Handlungsfelder 3 und 4 bei der Verteilung der Fördermittel in der Periode 1999 bis 2004 angemessener gewesen wäre.

\section{Wird die Umsetzung des Rahmenkonzepts effizient administriert?}

Die organisatorischen und administrativen Abläufe von der Bekanntmachung über die Skizzenauswahl bis zur Betreuung der geförderten Projekte sind klar strukturiert und in den internen QS Dokumenten des Projektträgers entsprechend dokumentiert. Die Arbeit des Projektträgers wird von den Programmteilnehmern aus Industrie und Forschung insgesamt ausgesprochen positiv beurteilt. Auch im Vergleich zu uns zur Verfügung stehenden Ergebnissen aus anderen Evaluationen erscheint die Bewertung des Projektträgers durch die Teilnehmer des Programms hervorragend. Als am häufigsten genannte Kritikpunkte im Verfahren der Antrags- und Projektabwicklung wird die Skizzenbewertung und -auswahl thematisiert. Diese lässt nach Angaben der Teilnehmer bezüglich Transparenz der Beurteilungskriterien und Nachvollziehbarkeit der Entscheidung über die Weiterverfolgung der Skizzen ein Verbesserungspotenzial erkennen. Im Hinblick auf die verfügbaren Informationen über geförderte Verbundprojekte im Internet fällt auf, dass sich diese häufig auf die technischen Aspekte der Vorhaben konzentrieren während die Vermarktungs- und Verwertungsdimension in den Beschreibungen häufig zu kurz kommt. Auch ist auf der InternetSeite des Projektträgers kein Zugriff auf die Ergebnisberichte der Verbundprojekte möglich.

\section{Handlungsempfehlungen}

Die im Rahmenkonzept formulierten Herausforderungen und Ziele sowie die zur Verfügung stehenden Instrumente sollten im Lichte der Evaluationsergebnisse reflektiert und gegebenenfalls angepasst werden. Dies betrifft beispielsweise den Anspruch, die breite Anwendung von FuE-Ergebnissen in KMU unterstützen zu wollen. Unser Vorschlag ist, das Wort „breit“" zu streichen.

Im Hinblick auf den Anspruch ein „lernendes Programm“ zu sein, sollte eine dokumentierte kontinuierliche Weiterentwicklung des Rahmenkonzepts bezüglich 
der Herausforderungen und Schwerpunkte der Förderung angestrebt werden. Ein mögliches Instrument dazu wäre eine in regelmäßigen Abständen (z.B. alle zwei Jahre) fortgeschriebene strategische Planung des Rahmenkonzepts mit der Erstellung einer mittelfristigen Programm-Roadmap. Dadurch könnte die Kohärenz zwischen Programmanspruch und Umsetzung jedenfalls gewährleistet werden.

Ein Monitoring- und Evaluationskonzept, in dem die Datenerfordernisse, die Methoden und aussagekräftige Indikatoren festgelegt sind, um den Programmfortschritt beurteilen zu können, könnte das BMBF und den Projektträger bei der Umsetzung des Rahmenkonzepts unterstützen. Bestandteile eines solchen Konzepts wären eine regelmäßige ex-post Evaluation in etwa fünfjährigen Abständen und das Nachzeichnen der Kommerzialisierungserfolge der geförderten Verbundvorhaben ex-post durch den Projektträger.

Der integrative Programmanspruch, der sich in den vier Handlungsfeldern des Rahmenkonzepts widerspiegelt, ist innovativ und nach wie vor stimmig. In der kommenden Programmperiode sollte verstärkt auf eine ausgewogene Berücksichtigung aller Handlungsfelder geachtet werden. Mehr horizontale Bekanntmachungen, also solche die mehrere Handlungsfelder gleichzeitig adressieren, erscheinen im Lichte der Programmzielsetzungen wünschenswert. Auch programmübergreifende Bekanntmachungen sollten häufiger in Betracht gezogen werden.

In der Programmperiode 1999 bis 2004 wurden vor allem Stückgut fertigende Betriebe aus höherwertigen Technologiebereichen des deutschen Verarbeitenden Gewerbes gefördert, die traditionell zu den Stärken der deutschen Wirtschaft gehören. Betriebe der Prozessindustrie sowie Betriebe aus Low-Tech-Sektoren wurden bislang mit dem Rahmenkonzepts kaum erreicht. Hier ist auch mit Blick auf die Portefeuilles anderer Fachprogramme zu klären, ob diese Schwerpunktsetzung richtig und strategisch gewollt ist.

Das Rahmenkonzept „Forschung für die Produktion von morgen“ könnte in Zukunft stärker strategisch dazu genutzt werden, deutsche FuE Politik im Bereich der Produktionsforschung intensiver in multilaterale, europäische oder auch bilaterale Kooperationen einzubringen. Dies könnte längerfristig positive Auswirkungen auf die stärkere Berücksichtigung deutscher FuE Schwerpunkte und Interessen in der internationalen Forschungszusammenarbeit zeigen, beispielsweise bei der Umsetzung von Manufuture.

Bei der Auswahl der Verbundprojekte sollten verstärkt hochinnovative Vorhaben, die ambitionierte neue Ansätze im Sinne des integrativen Anspruchs des Rahmenkonzepts verfolgen, besonders berücksichtigt werden. Der Integration von Anwendern und der Erstellung von geeigneten Markt- und Transferstrategien (mit festgelegten Zielen) sollte bei der Auswahl der Skizzen und im Vor-Projekt-Verfahren eine besondere Bedeutung zugemessen werden. Als Fördergrundsatz sollte gelten: In jedem geförderten Projekt muss die Nutzer- und Anwenderperspektive integriert sein!

Die Umsetzung des Programms wird vom Projektträger ausgesprochen gut administriert. Verbesserungspotenziale im Verfahren sehen wir noch in einer begründeten standardisierten Rückmeldung der Entscheidung über die Skizzen 
(ähnlich der derzeitigen EU Rahmenprogrammpraxis), um dadurch Lerneffekte zu ermöglichen und insgesamt die Transparenz des Verfahrens zu erhöhen. Um gleiche Voraussetzungen im Auswahlverfahren sicher zu stellen, sollten die weiterverfolgten Skizzen ausschließlich aus dem Kreis der im Auswahlgespräch präsentierten Vorhaben stammen. Die Ergebnisse der Auswahlverfahren sollten auf den InternetSeiten des Projektträgers veröffentlicht werden.

Die auf den Internet Seiten des Projektträgers zum Rahmenkonzept angebotenen Informationen über laufende und abgeschlossene Verbundprojekte erscheinen uns verbesserungswürdig. Die Projektendberichte sollten in Form von transfer- und verwertungsorientierten Broschüren zum direkten Herunterladen zur Verfügung stehen. Industriearbeitskreise könnten verstärkt entlang der Themenfelder des Rahmenkonzepts eingerichtet werden (und weniger entlang einzelner Verbundprojekte), um so das Interesse einer breiteren Klientel zu wecken. 


\section{Inhalt}

1 Einleitung........................................................................................................................1

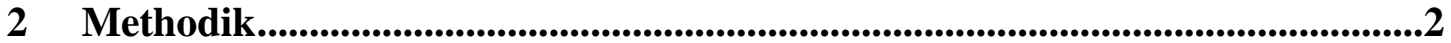

3 Produktionstechnische Wirtschaft und Wissenschaft ..........................................6

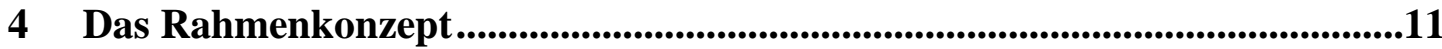

5 Kennzahlen zum Rahmenkonzept .................................................................15

5.1 Beteiligung an den Bekanntmachungen ................................................................ 15

5.2 Programmumsetzung nach Handlungsfeldern............................................................17

5.3 Angestrebte Innovationen in den geförderten FuE-Vorhaben........................................19

5.4 Analyse der Teilnehmerstruktur ..........................................................................................20

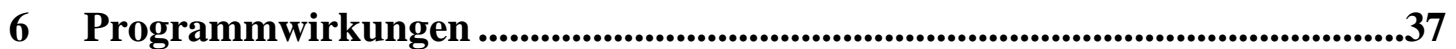

6.1 Additionalität und Anstoßwirkung der Projektförderung ..........................................37

6.2 Wirtschaftliche Verwertung der Projektergebnisse.......................................................42

6.3 Wirkung der Projektteilnahme auf die Kompetenzen der Betriebe und auf die Bewältigung von Innovationshemmnissen ............................................................................49

6.4 Hemmnisse beim Transfer von Projektergebnissen........................................................54

6.5 Anmerkungen zu Wirkungen im ungeförderten Bereich ..........................................57

7 Administrative Programmdurchführung........................................................60

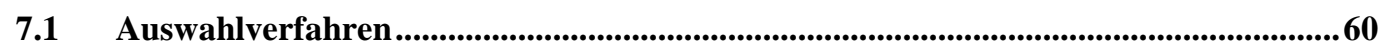

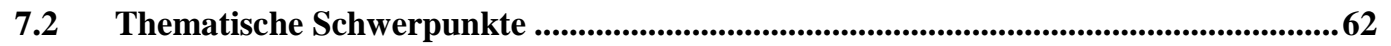

7.3 Beurteilung der Programmadministration durch die Teilnehmer ...............................63

7.4 Transfermaßnahmen und Öffentlichkeitsarbeit ......................................................64

8 Programmstrategie: Anregungen des Experten Panels ...................................67

9 Schlussfolgerungen ..........................................................................................71

9.1 Diskussion der Befunde der Evaluation ................................................................71

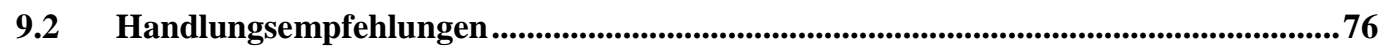

Appendix A Verzeichnis der Arbeitspaketberichte.............................................79

Appendix B Leistungsbeschreibung .........................................................................80 


\section{Abbildungsverzeichnis}

Abbildung 1 Anteil der FuE-Aufwendungen am Umsatz und FuE-Aufwand ....................... 9

Abbildung 2 Interner FuE-Aufwand an der Bruttowertschöpfung ...................................... 9

Abbildung 3 Verteilung der Fördermittel nach Handlungsfeldern ....................................... 18

Abbildung 4 Angestrebte Innovationen in den geförderten FuE-Vorhaben ......................... 19

Abbildung 5 Größenstruktur der beteiligten Industriepartner............................................ 24

Abbildung 6 Größenstruktur der beteiligten Betriebe des Verarbeitenden Gewerbes im Vergleich mit der Grundgesamtheit......................................... 25

Abbildung 7 Vergleich der Größenstruktur der geförderten Verarbeitenden

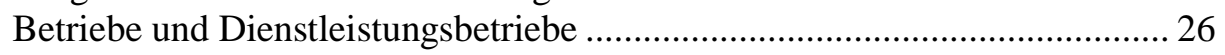

Abbildung 8 Geförderte Verarbeitende Betriebe aus den alten und neuen

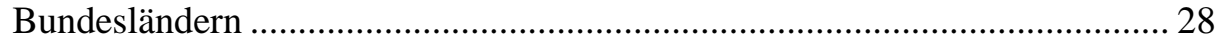

Abbildung 9 Institutsbeteiligung nach Fachbereichen..................................................... 33

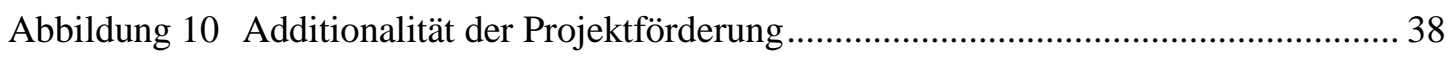

Abbildung 11 Weiterführung der Entwicklungen nach Projektabschluss .............................. 39

Abbildung 12 Weiterführung der Kooperationen in anderen Bereichen ............................... 40

Abbildung 13 FuE Additionalitäten der Förderung für alle Unternehmen und für die Teilgruppe der KMU.............................................................................. 41

Abbildung 14 Wirkung der Förderung bei Produktinnovationen ......................................... 43

Abbildung 15 Effekte der Förderung bei technischen Prozessinnovationen ........................... 46

Abbildung 16 Effekte der Förderung bei organisatorischen Innovationen ............................. 47

Abbildung 17 Einfluss der Projektergebnisse auf Kosten und Ertragsstärke ......................... 48

Abbildung 18 Patentierung und Lizenzierung von Projektergebnissen ................................. 48

Abbildung 19 Projektwirkungen auf den Märkten ............................................................. 49

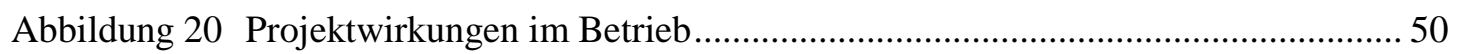

Abbildung 21 Wirkungen der Projektteilnahme nach Betriebsgröße ..................................... 52

Abbildung 22 Wirkungen der Projektteilnahme nach Innovationsgrad..................................53

Abbildung 23 Gründe, warum Ergebnisse nicht angewendet werden .................................. 54

Abbildung 24 Beurteilung der Antrags- und Projektabwicklung .......................................... 64

\section{Box Verzeichnis}

Box 1 Produktionstechnische Wirtschaft und Wissenschaft ..................................... 6

Box 2 Welche Gründe sind für KMU besonders relevant sich an

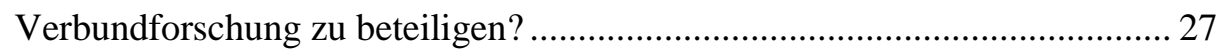

Box 3 Fallstudie ChopArc: Technologietransfer über Köpfe................................... 51

Box 4 Fallstudie OPAQ: Technische und organisatorische Innovationen

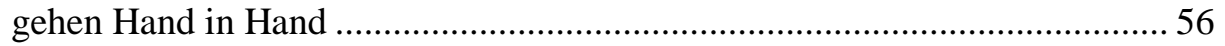

Box 5 Fallstudie ErGo: Herausforderungen Anwendung und Ergebnistransfer 


\section{Tabellenverzeichnis}

Tabelle 1 Übersicht über eingereichte Skizzen zu den Bekanntmachungen in

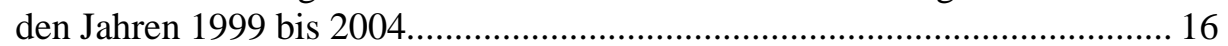

Tabelle 2 Verbundprojekte nach Handlungsfeld und Jahr........................................... 17

Tabelle 3 Verbundprojekte nach Innovationsorientierung und Jahr................................ 18

Tabelle $4 \quad$ Struktur der teilnehmenden Betriebe nach Branchen ...................................... 21

Tabelle 5 Struktur der teilnehmenden Betriebe aus dem Verarbeitenden Gewerbe im Vergleich zur Grundgesamtheit ................................................. 22

Tabelle 6 Förderung der Projektteilnehmer und Teilnahmen an Verbundvorhaben nach Projektrollen 31

Tabelle $7 \quad$ Vergleich der Institutsfördermittel nach Fachbereichen mit den Anteilen an den Drittmitteleinnahmen aller Fachbereiche ............................... 34

Tabelle 8 Effekte der Förderung bei produktbegleitenden Dienstleistungen .................. 44

Tabelle 9 Umsätze mit Produkten und Dienstleistungen, die unmittelbar auf den Vorhabensergebnissen aufbauen

Tabelle 10 Gründe, warum Entwickler und Zulieferer Ergebnisse der FuEVorhaben nicht anwenden 


\section{$1 \quad$ Einleitung}

Seit Ende 1999 fördert das BMBF mit dem Rahmenkonzept „Forschung für die Produktion von morgen" Forschung in Verbundprojekten mit dem Ziel, beispielhafte Lösungen für die zukunftsfähige Produktion in Deutschland zu entwickeln und Forschungsergebnisse für die breite Anwendung besonders für kleinere und mittlere Unternehmen (KMU) bereitzustellen. Das BMBF Rahmenkonzept wurde als lernendes Programm konzipiert, d.h. das Programm hat keine festgelegte Laufzeit, sondern die Programmstrategie und die Schwerpunkte sollen laufend an das sich verändernde Umfeld produzierender Unternehmen angepasst werden.

Nach einigen Jahren Laufzeit eines Förderprogramms bietet es sich an, die bisher erzielten Ergebnisse der durchgeführten Fördermaßnahmen im Rahmen einer Evaluation zu bewerten. Das BMBF hat dazu im Herbst 2004 einen Auftrag zur Durchführung der ex-post Evaluation des Programms „Forschung für die Produktion von morgen“ für die Jahre 1999 bis 2004 öffentlich ausgeschrieben. Technopolis wurde schließlich in Kooperation mit dem Fraunhofer-Institut für System- und Innovationsforschung (FhG-ISI) und ARÖW vom BMBF mit der Durchführung dieser Evaluation beauftragt.

Die Evaluation des Rahmenkonzepts „Forschung für die Produktion von morgen“ soll die Frage beantworten, welchen Beitrag dieses Programm bzw. sein zentrales Instrument der Verbundprojektförderung zur Stärkung der technologischen und wirtschaftlichen Wettbewerbsfähigkeit des deutschen Produktionssektors, insbesondere auch der kleinen und mittleren Unternehmen (KMU), leistet. Gemäß der Leistungsbeschreibung ist es Ziel der Evaluation des Programms „Forschung für die Produktion von morgen" der Jahre 1999-2004, die Wirkungen der Programmförderung vor dem Hintergrund der gesetzten Ziele darzustellen und zu bewerten, um so die Informationen zusammen zu tragen, die als Grundlage für politische Entscheidungen über das $\mathrm{Ob}$ und Wie der weiteren Förderung von Forschung im Bereich der Produktion benutzt werden können.

Zwei Aspekte sind dabei für die Evaluation zentral: Einerseits geht es um die Auswertung der Wirkungen der bisherigen Förderung des Programms in Bezug auf die gesetzten Ziele, im Sinne des ökonomischen und gesellschaftlichen Nutzens, der mit den eingesetzten Ressourcen im Programm bisher erzielt wurde. Dabei war insbesondere zu überprüfen, welche potenziellen Zielgruppen erreicht und welche nicht erreicht werden konnten, wobei besonderes Augenmerk auf den Nutzen des Programms für die Gruppe der KMU gelegt werden sollte. Darüber hinaus dient die Evaluation der Charakterisierung und Einschätzung der Status-quo-Situation von Produktion und Produktionsforschung in Deutschland. Es wurden dazu zentrale Kennziffern über die Struktur und Entwicklung von Produktion und Produktionsforschung in Deutschland in den Jahren 1999 bis 2004 ausgewertet.

Mit Hilfe der genannten Dimensionen der Evaluation sollen klare Aussagen darüber möglich werden, wo im Lichte der Programmzielsetzungen gegebenenfalls die neuralgischen Punkte liegen, an denen Verbesserungen des Programms in Zukunft sinnvoll ansetzen können. 


\section{Methodik}

Wie bereits erwähnt soll mit dieser Ex-post Evaluation schwerpunktmäßig geprüft werden, ob und in welchem Ausmaß die Ziele des Forschungsprogramms erreicht worden sind (Zielerreichungskontrolle), ob die Maßnahmen für die Zielerreichung ursächlich waren (Wirkungskontrolle) und ob sie sich als wirtschaftlich erwiesen haben (Wirtschaftlichkeitskontrolle). Die Ergebnisse der Evaluation sollen eine Einschätzung der erzielten Effekte des Programms ermöglichen bzw. erkannte Defizite aufzeigen. Auf Basis der Erhebungen und Auswertungen sollen dem Auftraggeber Empfehlungen für die Gestaltung künftiger Fördermaßnahmen für das produzierende Gewerbe in Deutschland gegeben werden.

Das Verständnis des Programmkontextes und der Zusammenhänge mit den zentralen Evaluationsdimensionen, das wir dieser Evaluation zugrunde legen, ist in der folgenden Abbildung schematisch zusammengefasst.

\section{Abbildung 1 Evaluationsdimensionen im Programmkontext}

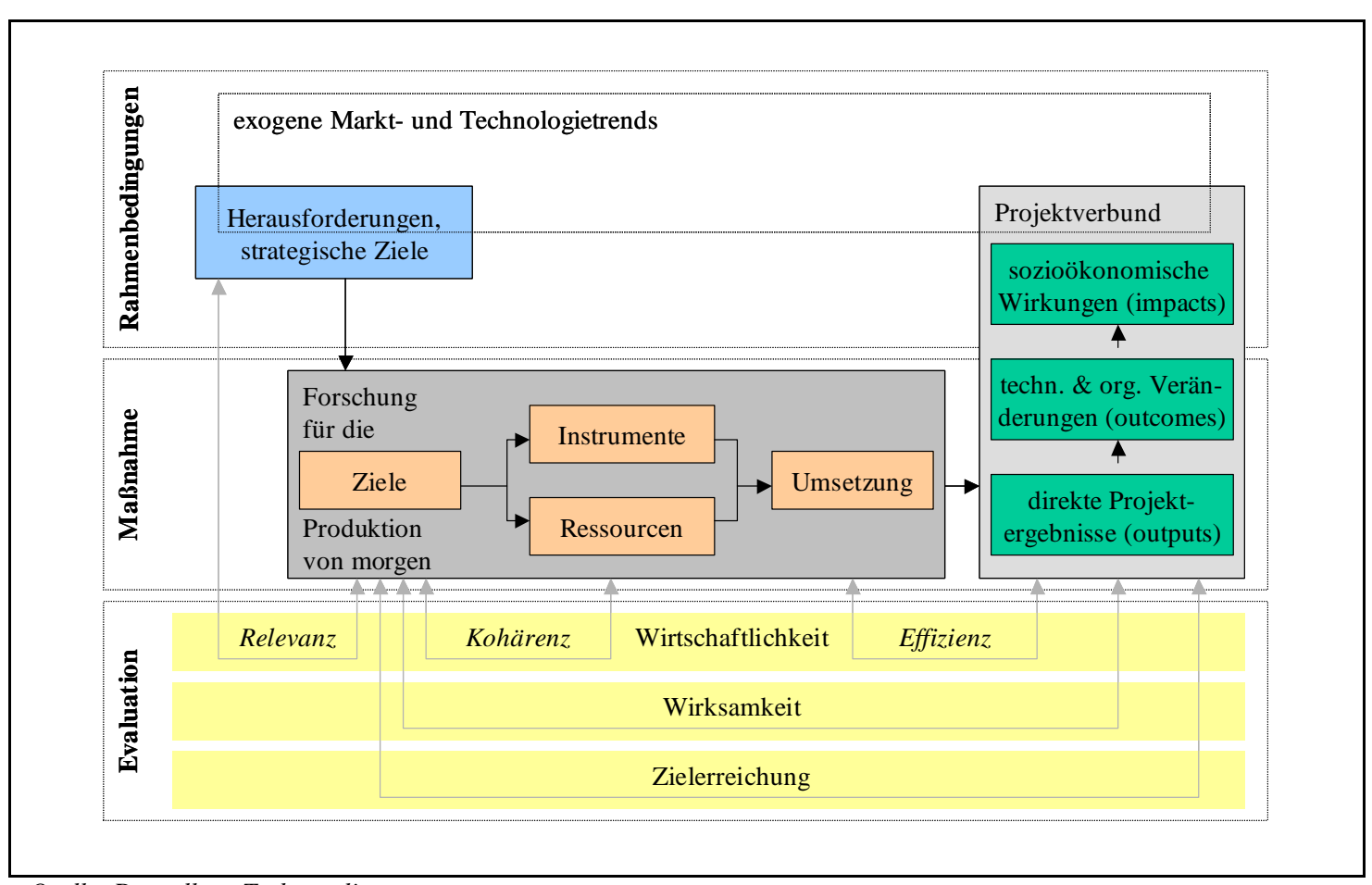

Quelle: Darstellung Technopolis

Für die Durchführung der Evaluation haben wir einen umfassenden Mix aus qualitativen und quantitativen sozialwissenschaftlichen Evaluierungsmethoden eingesetzt, um der Vielschichtigkeit der Evaluationsaufgabe möglichst optimal gerecht zu werden. Konkret wurden im Rahmen der Durchführung der Evaluation die folgenden methodischen Elemente eingesetzt. Die Arbeiten wurden von uns in neun Arbeitspaketen strukturiert.

\section{Arbeitspaket 1: Dokumentenanalyse}

Die öffentlich verfügbaren Programmdokumente über das Rahmenkonzept „Forschung für die Produktion von morgen“ sowie uns zusätzlich vom BMBF bzw. vom Projektträger zur Verfügung gestellten schriftlichen Informationen über das 
Programm bzw. seine Durchführung in den Jahren 1999 bis 2004 wurden von uns ausgewertet. Alle beim Projektträger verfügbaren Projektrahmenpläne der in der Evaluationsperiode 1999 bis 2004 gestarteten Verbundprojekte wurden von uns zur Klassifizierung der Rollen der Projektteilnehmer im Verbund herangezogen. Auch die Verbünde selbst wurden entlang der Dimensionen Innovationstyp, Entwicklungsziel und Endergebnis charakterisiert. Für eine Stichprobe von $160 \mathrm{KMU}$, deren Projekte zum Ende des Jahres 2004 bereits abgeschlossen waren, wurden von uns die Schluss- und Erfolgskontrollberichte nach den Dimensionen Erfahrungen im Verbundprojekt, erzielte Projektergebnisse und Verwertungsperspektiven, Verbreitung der Ergebnisse - Transfer für Dritte, Vorteile und Nachteile des Instruments Verbundprojekt und Beurteilung der Betreuung durch den Projektträger ausgewertet. Die Dokumentenanalyse wurde von uns im Herbst 2005 zu einem beträchtlichen Anteil Vor-Ort beim Projektträger in Karlsruhe bzw. in der Außenstelle in Dresden durchgeführt. Dadurch konnten auftauchende Fragen zu einzelnen Verbundprojekten bzw. Projektpartner direkt mit den zuständigen BetreuerInnen des Projektträgers geklärt bzw. diskutiert werden.

\section{Arbeitspaket 2: Klientelanalyse (Auswertung der Förderdaten)}

Der Projektträger stellte uns einen Datenauszug aus dem BMBF ProjektförderInformationssystem (PROFI) mit Basisinformationen zu den geförderten Organisationen des Programms in den Jahren 1999 bis 2004 zur Verfügung. Fehlende Angaben im Datensatz, insbesondere zur Betriebs- bzw. Unternehmensgröße wurden von uns unter Zuhilfenahme anderer Datenquellen (Hoppenstedt Firmendatenbank) ergänzt. Der PROFI Förderdatensatz wurde von uns mit dem Auswertungsraster für die Rolle der Projektpartner in den Verbundprojekten verknüpft. Damit konnten die Auswertungen zur Klientelanalyse und der on-line Befragung (siehe Arbeitspakete 4 und 5) auch nach Projektrolle differenziert durchgeführt werden. Für die Klientelanalyse konnten von uns auch Vergleiche auf Grundlage der Ergebnisse der FhG-ISI Befragungen „Innovationen in der Produktion“ der Jahre 2001 und 2003 durchgeführt werden.

\section{Arbeitspaket 3: Auswertung verfügbarer statistischer Sekundärdatenquellen zum Status Quo und der Entwicklung der produktionstechnischen Wirtschaft und Wissenschaft in Deutschland 1999 bis 2004}

Für die Erhebung des Status Quo und die Entwicklung der produktionstechnischen Wirtschaft und Wissenschaft in Deutschland in den Jahren 1999 bis 2004 wurden von uns verfügbare statistische Sekundärdatenquellen herangezogen. Insbesondere stützen wir unsere Analysen und Vergleiche auf Daten der OECD, des Statistischen Amts der Europäischen Gemeinschaften (EUROSTAT), des Statistischen Bundesamts, des Stifterverbands für die Deutsche Wissenschaft sowie auf eine Reihe vorliegender Analysen und Studien zur FuE und Innovation im deutschen produzierenden Gewerbe.

\section{Arbeitspaket 4: Durchführung einer quantitativ empirischen Wirkungsanalyse}

In einem Kontrollgruppenvergleich über den Matched-Pair Ansatz, der auch bei vergleichsweise kleinen Stichproben statistisch robuste Ergebnisse liefern kann, haben wir mit den Umfragedaten aus den FhG-ISI-Erhebungen der Jahre 2003 und 2006 versucht, eine mögliche FuE-Anstoßwirkung der Förderung zu identifizieren 
und erste ökonomische Effekte (wie z.B. Produktinnovationen, produktbegleitende Dienstleistungen, technische Prozessinnovationen und organisatorische Innovationen) zu erfassen. Mit dem Matched-Pair-Ansatz können im Vergleich zwischen geförderten und nicht geförderten Betrieben für den FuE-Aufwand bzw. mögliche ökonomischen Effekte relevante, intervenierende Einflussfaktoren kontrolliert werden.

\section{Arbeitspaket 5: Durchfïhrung einer on-line Befragung}

Insgesamt standen uns für die Befragung die e-mail Adressen von 1153 Projektleitern und Projektleiterinnen der Einzelvorhaben aus der PROFI Datenbank zur Verfügung. Darunter befanden sich 891 Industriepartner und 262 Institutspartner. Die Befragung wurde zwischen 12. Januar 2006 und 10. Februar 2006 on-line durchgeführt. Dazu wurde von uns die Webseite www.evaluation-produktionsforschung.de eingerichtet. Die Bereitschaft zur Beteiligung an der Befragung war ungewöhnlich hoch. Insgesamt konnten für die Auswertung die Datensätze für 593 FuE-Vorhaben (davon 427 Industriepartner und 166 Institutspartner) herangezogen werden. Dies entspricht einem verwertbaren Rücklauf von 51,4\% (47,9\% bei Industriepartnern und 63,4\% bei Institutspartnern).

\section{Arbeitspaket 6: Qualitative Interviews}

Mit rund 50 Personen aus dem Programm und seinem Umfeld wurden von uns Gespräche geführt. Unsere GesprächspartnerInnen kamen sowohl aus der Gruppe der Projektpartner aus auch von Teilnehmer an Industriearbeitskreisen, sowie Vertretern von Verbänden und wissenschaftlichen Einrichtungen. Wir sprachen auch mit einzelnen Mitgliedern des Gesprächskreises, Strategien für die Produktion des 21. Jahrhundert' und natürlich mit den Programmverantwortlichen und zahlreichen ProjektbetreuerInnen beim Projektträger.

\section{Arbeitspaket 7: Durchführung von Verbundprojekt-Fallstudien}

Im Rahmen von drei Verbundprojekt-Fallstudien wurden von uns 15 Projektpartner detailliert über ihre Vorhaben befragt. Die Auswahl der Fallstudien wurde so gestaltet, dass sowohl technisch als auch nicht-technisch orientierte Verbundprojekte berücksichtigt werden konnten. Der Schwerpunkt in den Gesprächen lag bei der Verwertung der Projektergebnisse nach Ende der Verbundvorhaben. Es ging uns vor allem darum zu erfahren, welche praktischen Konsequenzen auf technischer, organisatorischer, strategischer und wirtschaftlicher Ebene die Beteiligung am Verbundprojekt für die beteiligten Einrichtungen (und möglicher weise für Dritte) hatte.

\section{Arbeitspaket 8: Auswertung der Karlsruher Arbeitsgespräche Produktions- forschung der Jahre 2000, 2002 und 2004 und Durchführung einer Befragung bei den Karlsruher Arbeitsgesprächen 2006}

Insbesondere ging es uns darum, Hinweise zu finden, inwieweit durch die Karlsruher Arbeitsgespräche Impulse für die Entwicklung von neuen thematischen Schwerpunkten des Rahmenkonzepts gegeben werden, in wie weit die Gespräche eine Plattform für Wissensaustausch, die Initiierung neuer Geschäftsbeziehungen und Kontakte bieten, in welchem Umfang die Gespräche einen Beitrag zur Verbreitung 
und zum Transfer der Forschungsergebnisse liefern können und in wie weit mit den Karlsruher Arbeitsgesprächen KMU und Akteure aus den Neuen Bundesländern erreicht werden konnten.

\section{Arbeitspaket 9: Einrichtung eines Experten Panels}

Eine Gruppe von sieben internationalen Experten aus der Produktions- und Innovationsforschung wurde von uns eingeladen, die Strategie und die thematische Umsetzung des BMBF Rahmenkonzepts in den Jahren 1999 bis 2004 im Lichte ihrer internationalen Erfahrung kritisch zu kommentieren. Im Vorfeld wurden von uns dazu auch verfügbare Informationen und Programmdokumente zur Förderung von produktionstechnischer Forschung außerhalb Deutschlands ausgewertet. Die Experten gaben auf Grundlage der zur Verfügung gestellten Dokumente zuerst jeweils ihre individuellen schriftlichen Einschätzungen des Rahmenkonzepts ab, die in ein gemeinsames Arbeitsdokument kondensiert wurden. Dieses wurde dem $\mathrm{BMBF}$ und dem Projektträger zur Verfügung gestellt und diente als Grundlage für einen gemeinsamen Panel Workshop der am 26. September 2006 am BMBF in Bonn stattfand. Die Ergebnisse dieses Workshops flossen in die endgültige Stellungnahme des Experten Panels ein. Die Einschätzungen und Vorschläge der Experten wurden in diesem Bericht berücksichtigt.

\section{Arbeitspaketberichte}

$\mathrm{Zu}$ jedem einzelnen der oben angeführten Arbeitspakete wurden von uns schriftliche Berichte verfasst. Alle Arbeitspaketberichte wurden dem Auftraggeber als Hintergrundinformationen zur Verfügung gestellt. Die Berichte zu den Arbeitspaketen Dokumentenanalyse, Klientelanalyse und Qualitative Interviews wurden zudem im Rahmen der Präsentation der Zwischenergebnisse der Evaluation im Februar 2006 mit dem Auftraggeber diskutiert. Die vollständige Liste der Berichte zu den neun Arbeitspaketen findet sich in Appendix A zu diesem Bericht.

Die Arbeitspaketberichte beleuchten das Programm von unterschiedlichen Perspektiven. Sie dienten dem Evaluationsteam auch dazu, aus der jeweiligen Perspektive Interpretationen über das Rahmenkonzept zu versuchen und erste Vorschläge für spätere Handlungsempfehlungen auch intern abzutesten. Für die Beantwortung der uns gestellten Evaluationsfragen flossen die Ergebnisse der Diskussionen zu den einzelnen Arbeitspaketen in die in diesem Endbericht gemachten Aussagen und Handlungsvorschläge ein. 


\section{Produktionstechnische Wirtschaft und Wissenschaft}

Bevor wir uns dem Rahmenkonzept und seiner Umsetzung in den Jahren 1999 bis 2004 zuwenden, möchten wir den Blick auf den Status Quo und die Entwicklung der produktionstechnischen Wirtschaft und Wissenschaft in Deutschland lenken. Ein ausführlicher internationaler Vergleich der Entwicklung zentraler Indikatoren für die Leistungsfähigkeit der deutschen produzierenden Wirtschaft in der Periode 1999 bis 2004, besonders mit wichtigen Wettbewerbern findet sich im Bericht zum Arbeitspaket 3 dieser Evaluation.

\section{Box 1: Produktionstechnische Wirtschaft und Wissenschaft}

Das Rahmenkonzept zeichnet sich durch eine sehr breite Zielgruppe aus. In erster Linie werden durch das Programm Unternehmen des Verarbeitenden Gewerbes angesprochen (NACE Abschnitt D, Wirtschaftszweige 15 bis 37). Für diese Gruppe von Unternehmen verwenden wir in diesem Bericht den Begriff ,produktionstechnische Wirtschaft“ synonym. Unter den Branchen des Verarbeitenden Gewerbes werden durch die Orientierung des Rahmenkonzepts einige besonders stark angesprochen. Vor allem sind dies Unternehmen aus den Wirtschaftszweigen 28 bis 35 (Metallerzeugnisse, Maschinenbau, Elektrotechnik, Medizin-, Mess-, Steuer-, Regelungstechnik, Optik sowie Fahrzeugbau). Dort wo wir im Bericht spezifisch auf diese Kernbranchen eingehen, subsumieren wir diese Gruppe von Unternehmen als „Fertigungstechnik“.

Die alleinige Berïcksichtigung des Verarbeitenden Gewerbes als die industrielle Zielgruppe des Programms würde allerdings den Ansprüchen des Rahmenkonzepts nicht gerecht werden. Auch Unternehmen aus dem Dienstleistungsbereich und hier insbesondere Unternehmen, die den Wirtschaftszweigen WZ 72: Datenverarbeitung und Datenbanken, WZ 73: Forschung und Entwicklung und WZ 74: Erbringung von Dienstleistungen für Unternehmen zugeordnet sind werden vom Rahmenkonzept angesprochen und werden - wie wir später zeigen werden - in den Verbundprojekten des Rahmenkonzepts auch in relevantem Umfang integriert. Diese Unternehmen werden im Folgenden unter dem Begriff ,,produktionsnahe Dienstleistungen“ zusammengefasst.

Für eine sinnvolle Abgrenzung der „,produktionstechnischen Wissenschaft“ ziehen wir deren Relevanz für die produktionstechnische Wirtschaft heran. Eine Fokussierung auf die Ingenieurwissenschaften ist damit zu eng gefasst, denn auch naturwissenschaftliche, wirtschafts- und sozialwissenschaftliche Institute können zentrale Beiträge zur Produktionsforschung leisten. Allerdings ist die Abgrenzung relevanter und weniger relevanter Bereiche in diesen Wissenschaftsdisziplinen sehr schwierig. Zudem sind statistisch Daten zu spezifischen Wissenschaftsgebieten für die uns interessierenden Fragen im Rahmen dieser Evaluation nicht verfügbar. Bei den Analysen zur ,produktionstechnischen Wissenschaft" beschränken wir uns daher primär auf den ingenieurwissenschaftlichen Bereich.

\section{Zur Bedeutung und Dynamik der produktionstechnischen Wirtschaft}

Das Verarbeitende Gewerbe ist für die volkswirtschaftliche Leistungskraft Deutschlands von zentraler Bedeutung. Es stellt mit über 6 Mio. Personen ca. $37 \%$ aller Beschäftigten der privatwirtschaftlichen Wirtschaftszweige und trägt etwa $42 \%$ zur Bruttowertschöpfung und etwa $69 \%$ zum Produktionsvolumen dieser Sektoren bei. Zusammen mit den für das Rahmenkonzept ebenfalls relevanten produktionsnahen 
Dienstleistungen beschäftigen diese Sektoren damit fast $60 \%$ der ArbeitnehmerInnen der deutschen Wirtschaft (Wirtschaftszweige $\mathrm{C}$ bis $\mathrm{K}$, also ohne Land- und Forstwirtschaft und den öffentlichen Bereich) und erwirtschaften knapp 80\% des Produktionswertes. Die Exporte des Verarbeitenden Gewerbes umfassen ca. $97 \%$ des gesamten deutschen Exports. Eine besondere Stellung innerhalb des Verarbeitenden Gewerbes nehmen die fertigungstechnischen Unternehmen ein, die gut $60 \%$ der Exporte der deutschen Wirtschaft tätigen. Der Anteil der KMU im Verarbeitenden Gewerbe und in den produktionsnahen Dienstleistungssektor ist mit $96 \%$ bzw. fast $100 \%$ insgesamt sehr hoch, wobei die Sektoren Herstellung von Metallerzeugnissen und Medizin-, Mess-, Steuer-, Regelungstechnik, Optik die höchsten KMU-Anteile aufweisen.

Das deutsche Verarbeitende Gewerbe weist ein hohes durchschnittliches Wachstum (1999 bis 2003) beim Export sowie ein mittleres Wachstum beim Import und den FuE-Aufwendungen auf. Geringe Abnahmen sind bei den Beschäftigtenzahlen festzustellen, während das Produktionsvolumen angestiegen ist. Dies deutet darauf hin, dass sich die (Arbeits-)Produktivität in diesem Zeitraum deutlich positiv entwickelt hat. Insgesamt kann festgestellte werden, dass das deutsche Verarbeitende Gewerbe hinsichtlich der Indikatoren Exporterfolg und Produktivitätsfortschritt im Beobachtungszeitraum an Wettbewerbsfähigkeit gewonnen hat. Insbesondere die Unternehmen der Fertigungstechnik haben zwischen 1999 und 2003 zunehmend Investitionen getätigt, und zwar nicht nur in Anlagen und Geräte, sondern auch in Forschung und Entwicklung (FuE). Damit gehen relevante Steigerungen beim Import und insbesondere beim Export einher. Auffällig ist, dass sich die Beschäftigung in den fertigungstechnischen Unternehmen im Betrachtungszeitraum nur in sehr geringem Ausmaß reduziert hat.

Die produktionsnahen Dienstleistungen weisen in unserer Betrachtungsperiode die größte Dynamik auf. Sowohl bei den Beschäftigtenzahlen als auch bei FuE-Ausgaben und beim Handel sind vergleichsweise hohe Wachstumsraten zu verzeichnen. Die Veränderungen im Export und Import in diesem Bereich lassen Rückschlüsse auf einen relativ dynamischen Markt zu, der aber hinsichtlich seines absoluten Handelsvolumens noch relativ unbedeutend ist. Innerhalb der produktionsnahen Dienstleistungen zeichnen sich die FuE-Dienstleister durch eine besonders hohe Dynamik aus. Zwischen 2000 und 2003 sind ihre Beschäftigtenzahlen, die Unternehmenszahl sowie der Umsatz mit durchschnittlich zweistelligen Wachstumsraten angestiegen. Aber auch bei den Unternehmen der Branche Datenverarbeitung und Datenbanken ist bei diesen Indikatoren eine deutliche und hohe Dynamik im zweistelligen Bereich zu erkennen. Neben ihrer Dynamik ist die Datenverarbeitung auch aufgrund ihres Volumens unter den Dienstleistungsbranchen von Bedeutung, wobei unter den produktionsnahen Dienstleistungsbereichen eindeutig die Erbringung von Dienstleistungen für Unternehmen volumenmäßig die größte Rolle spielt, bei den FuE-Ausgaben aber den geringsten Anteil beisteuert.

Bemerkenswert ist, dass sich der Produktionswert im Verarbeitenden Gewerbe weiterhin positiv entwickelt und in den fertigungstechnischen Branchen die Beschäftigungsentwicklung nicht signifikant negativ ist. Dies deutet darauf hin, dass der Strukturwandel in Deutschland nicht nur zwischen den Sektoren verläuft, sondern ein Teil der wissensintensiven Dienstleistungen für das Verarbeitende Gewerbe auch weiterhin in diesem Sektor stattfindet. Diese innere Tertiarisierung 
scheint insbesondere in der Fertigungstechnik ausgeprägt zu sein. Zudem scheinen die in einzelnen Branchen der Fertigungstechnik gängigen Auslagerungspraktiken bislang nur zu einem Abschmelzen der internen Produktion und Beschäftigung in einem Ausmaß geführt zu haben, das durch externes Wachstum, insbesondere aus der zunehmenden Exporttätigkeit, nahezu kompensiert (siehe Beschäftigung) bzw. überkompensiert (siehe Produktion) werden konnte. Ein Teil des Strukturwandels zur Dienstleistungs- und Wissensgesellschaft findet demnach wohl innerhalb des produzierenden Sektors selbst statt und ist daher statistisch auf einfache Weise nicht nachvollziehbar. Insgesamt zeigt sich aber, dass sich das deutsche Verarbeitende Gewerbe und insbesondere die Fertigungstechnik in nahezu allen herangezogenen Indikatoren in den vergangenen Jahren sehr positiv entwickelt haben.

\section{FuE in der produktionstechnischen Wirtschaft}

Die FuE-Ausgaben der deutschen Wirtschaft konzentrieren sich seit Jahrzehnten recht stark auf einige ausgewählte Industriezweige. Ungefähr $90 \%$ der FuEAufwendungen entfallen auf das Verarbeitende Gewerbe, wobei allein die fertigungstechnischen Unternehmen über $80 \%$ der privatwirtschaftlichen FuEAufwendungen erbringen. Alle Kernbranchen des deutschen Verarbeitenden Gewerbes haben zwischen 1999 und 2003 ihre gesamten wie auch ihre intern erbrachten FuE-Aufwendungen in der absoluten Höhe steigern können.

Diese Konzentration und positive Dynamik ist vor allem auf den Fahrzeugbau zurückzuführen, dem als international anerkannter Vorreitermarkt traditionell eine hohe Bedeutung für die deutsche Wirtschaft zukommt. Die relative Bedeutung dieses Sektors für die FuE-Aktivitäten der deutschen Wirtschaft ist in den letzten Jahren weiter gestiegen. Doch auch andere Sektoren wie beispielsweise der Maschinenbau oder die Hersteller von Büromaschinen, Datenverarbeitungsgeräten, Elektrizitätsgeräten, Rundfunk- und Nachrichtentechnik sowie Medizin-, Mess-, Steuer-, Regelungstechnik, Optik (NACE 30 bis 33) haben wachstumsbedingt ihre Anteile an den FuE-Aufwendungen der deutschen Wirtschaft etwas ausbauen können.

Die FuE-Aktivitäten der deutschen Wirtschaft sind trotz volumenmäßiger Konzentration verhältnismäßig breit gestreut, und verschiedene Sektoren weisen eine vergleichsweise hohe FuE-Intensität auf. Gemessen am Anteil der gesamten FuEAufwendungen (intern und extern) am Umsatz sind insbesondere der Fahrzeugbau $(6,9 \%)$, die Chemische Industrie $(7,4 \%)$ sowie der gesamte Bereich der Hersteller von Büromaschinen, Datenverarbeitungsgeräten, Elektrizitätsgeräten, Rundfunkund Nachrichtentechnik sowie Medizin-, Mess-, Steuer-, Regelungstechnik, Optik (NACE 30 bis 33 mit 7,4 \%) sehr FuE-intensiv. Auch der Maschinenbau (3,9\%) weist im Vergleich zum gesamten Verarbeitenden Gewerbe $(2,4 \%)$ noch eine überdurchschnittliche FuE-Intensität auf, jedoch schon mit deutlichem Abstand zu den zuvor angeführten Branchen. Allerdings konnte der Fahrzeugbau, der mit knapp 19 Mrd. EUR den deutlich größten und wachsenden Absolutbetrag für FuE aufwendet, seine FuE-Intensität nicht noch weiter steigern. Dagegen zeigt sich von 1999 bis 2003 in der Chemischen Industrie (von 6,7 \% auf 7,4 \%), in der Elektronik und Optik (NACE 30 bis 33, von 6,7 \% auf 7,4 \%) wie auch im Maschinenbau (von $3,3 \%$ auf $3,9 \%$ ) eine weiterhin zunehmende FuE-Intensität. 
Abbildung 1 Anteil der FuE-Aufwendungen am Umsatz und FuE-Aufwand

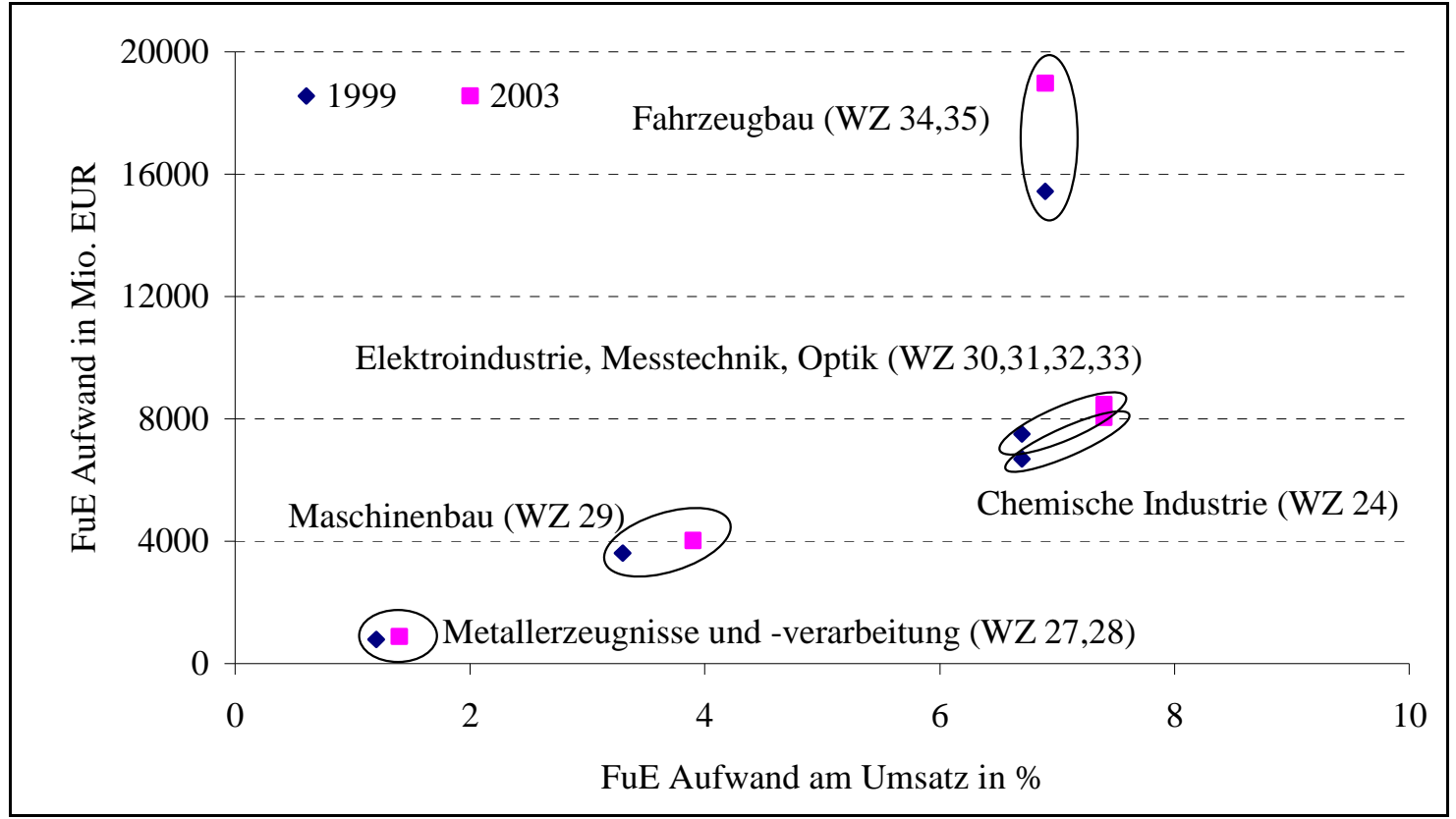

Quelle: Stifterverband für die deutsche Wissenschaft, Berechungen FhG-ISI

Der Anteil der internen FuE-Aufwendungen an der Bruttowertschöpfung zeigt zudem, dass Forschung und Entwicklung in den fertigungstechnisch relevanten Branchen noch immer stark in den Betrieben selbst verankert ist und nur zum Teil an spezialisierte Dienstleistungsunternehmen ausgelagert wurde. So liegt die absolute Höhe der internen FuE-Aufwendungen im Vergleich zu den gesamten (internen und externen) FuE-Aufwendungen in den betrachteten Branchen zwischen $75 \%$ im Fahrzeugbau und $93 \%$ im Maschinenbau. Nur im Fahrzeugbau und in der Chemischen Industrie (79\%) unterschreitet die ,interne Entwicklungstiefe“ die $80 \%$-Marke.

Abbildung 2 Interner FuE-Aufwand an der Bruttowertschöpfung

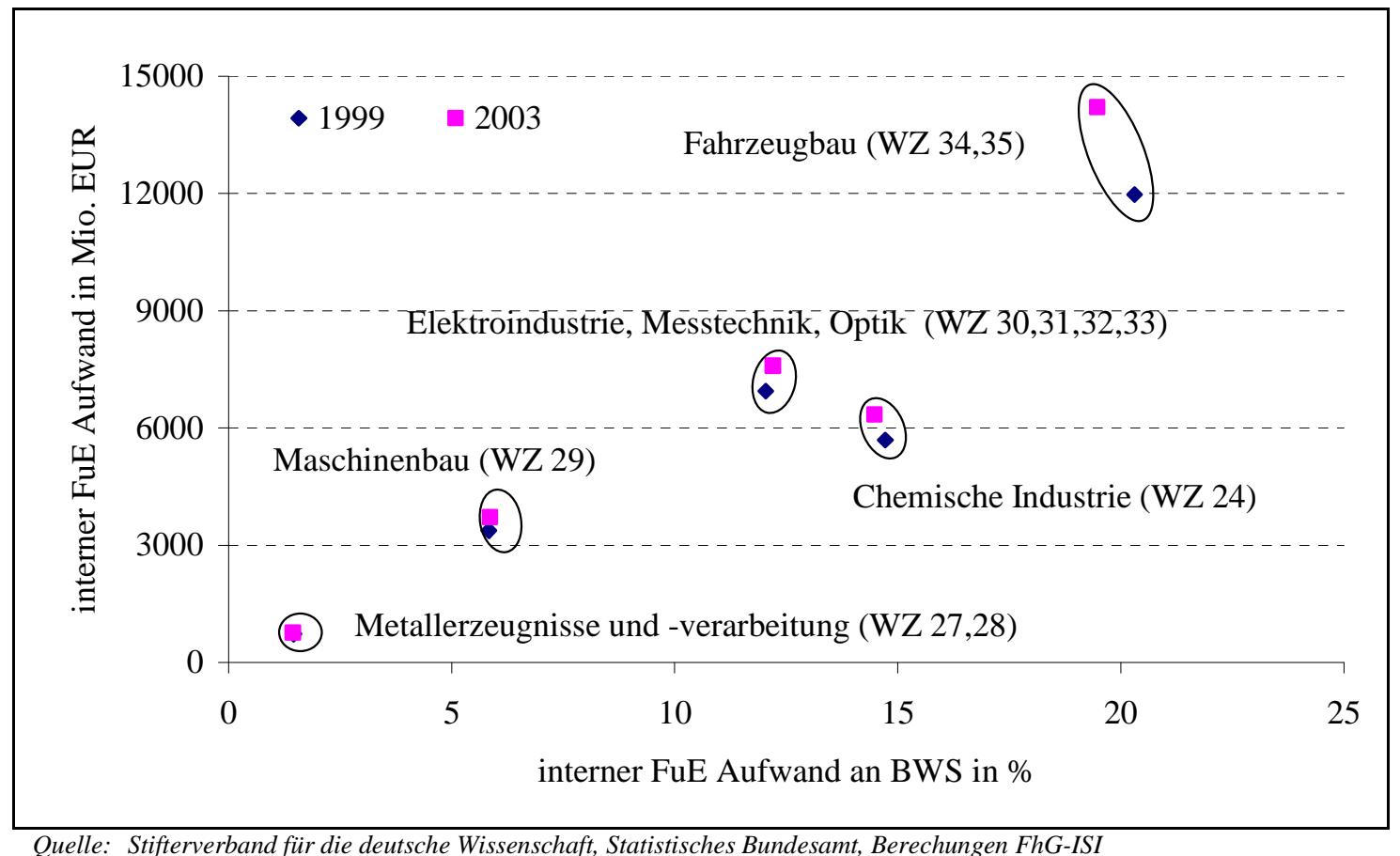


Ein Blick auf die Dynamik zeigt aber, dass der Anteil der internen FuE-Aufwendungen an der Bruttowertschöpfung zwischen 1999 und 2003 in den meisten Branchen stagniert oder leicht abgenommen hat. Der anteilige Zuwachs der gesamten internen wie externen FuE-Aufwendungen am Umsatz in den Branchen Maschinenbau, Elektronik und Optik sowie Chemische Industrie scheint demnach insbesondere durch die Vergaben von FuE-Aufträgen an andere Unternehmen vollzogen worden zu sein.

\section{Entwicklung der produktionstechnischen Wissenschaft}

Technisches Wissen wird von verschiedenen Akteuren geschaffen. Im staatlichen Sektor sind insbesondere die Hochschulen und die außeruniversitären Einrichtungen an der Wissensgenerierung beteiligt. Zusätzlich bilden die Hochschulen junge qualifizierte AbsolventInnen für die Industrie aus. Für die produktionstechnischen Unternehmen spielen vor allem die Ingenieurwissenschaften eine zentrale Rolle. Auch die Bereiche Informatik und Naturwissenschaften, insbesondere Physik und Chemie, sowie Wirtschafts- und Sozialwissenschaften sind in Teildisziplinen für die produktionstechnische Wirtschaft und Produktionsforschung insgesamt wichtig. In der Folge betrachten wir allerdings nur die Ingenieurwissenschaften.

Die deutschen Ingenieurwissenschaften vereinen etwa $21 \%$ des gesamten FuEBudgets der Universitäten und Fachhochschulen auf sich und haben für ihre FuEArbeiten auch $21 \%$ der durch die Wirtschaft finanzierten Drittmittel zur Verfügung. In Deutschland arbeiten etwa 18.600 Personen in der FuE der ingenieurwissenschaftlichen Hochschulinstitute. Der Anteil des FuE-Personals liegt bei knapp 19\%, allerdings haben sowohl die Anzahl als auch der Anteil des FuE-Personals in den vergangenen Jahren in diesem Fachbereich abgenommen.

Die Anzahl der AbsolventInnen im Fachbereich Ingenieurwesen gibt einen Anhaltspunkt, welchen Stellenwert die Ingenieurausbildung hat und welches Potenzial an hoch qualifizierten Arbeitskräften in technischen Produktionsberufen jährlich den Unternehmen in etwa zur Verfügung gestellt wird. In Deutschland gibt es jährlich in den Ingenieurwissenschaften etwa 52.000 AbsolventInnen, das entspricht ca. 17\% aller HochschulabsolventInnen. Allerdings ist sowohl die absolute Anzahl der ingenieurwissenschaftlichen Abschlüsse (-2\%) als auch ihr Anteil an allen Abschlüssen (-1,3 \%-Punkte) in den Jahren 1999 bis 2003 zurückgegangen. So haben 2003 etwa 4.500 weniger AbsolventInnen ein ingenieurwissenschaftliches Hochschulstudium abgeschlossen als noch im Jahr 1999. Im Hinblick auf die hohe Bedeutung des Verarbeitenden Gewerbes im allgemeinen und der Fertigungstechnik im besonderen setzt diese Entwicklung für die Industrie sicherlich kein positives Signal. 


\section{Das Rahmenkonzept}

In diesem Abschnitt beschreiben wir die Programmstrategie und die Schwerpunkte des BMBF Rahmenkonzepts „Forschung für die Produktion von morgen“ in den Jahren 1999 bis 2004. Das Rahmenkonzept geht davon aus, dass auch in Zukunft das produzierende Gewerbe den Kern der wirtschaftlichen Leistungsfähigkeit Deutschlands sein wird. Gleichzeitig argumentiert das Rahmenkonzept, dass die Rahmenbedingungen für Produktion einem grundlegenden Veränderungsprozess unterworfen sind, der Produzenten in Deutschland vor neue Herausforderungen stellt.

\section{Leitbild}

Als Stichworte zur Charakterisierung dieses Wandels werden angeführt:

- die rapide Ausbreitung neuer Technologien, besonders im Informations- und Kommunikationsbereich

- das Auftreten neuer, offensiver Wettbewerber

- eine - zumeist als Globalisierung bezeichnete - immer dichtere Vernetzung der weltwirtschaftlichen Güter-, Informations- und Kapitalströme

- tief greifende Veränderungen im Käuferverhalten und nicht zuletzt

- die zunehmend erkannte Notwendigkeit eines nachhaltigen, auf Dauer tragfähigen Wirtschaftens

Das BMBF will mit seinem Rahmenkonzept ,Forschung für die Produktion von morgen' die deutsche produzierende Industrie dabei unterstützen, diese Herausforderungen zu meistern und formuliert als Leitbild, dass durch aktive Gestaltung des technologischen, sozialen und ökologischen Wandels mit dem Ziel, Produkte und Dienstleistungen mit hohem Kundenutzen anzubieten, Unternehmen Technologie- und Marktführerschaft gewinnen und langfristig erfolgreich sein können.

\section{Handlungsfelder für Forschungsthemen im Rahmenkonzept}

Das Rahmenkonzept „Forschung für die Produktion von morgen“steht in einer langen Tradition der Förderung von Produktionsforschung und baut insbesondere auf den Ergebnissen des Vorläuferprogramms „Produktion 2000“ (1995 - 1999) auf. Zur Unterstützung der Programmentwicklung und zur Identifizierung des Handlungsbedarfs wurde im Jahr 1997 durch das BMBF eine Voruntersuchung mit dem Titel ,Produktion 2000plus' initiiert, in der mehr als 100 Repräsentanten der Industrie, der Tarifvertragspartner, der Forschung und der Fachverbände in Arbeitsgruppen mitarbeiteten.

Im BMBF Rahmenkonzept „Forschung für die Produktion von morgen“ wurden die erarbeiteten Handlungsempfehlungen berücksichtigt. Die vier Handlungsfelder des Programms entstammen der Voruntersuchung und die darunter beschriebenen ausgewählten thematischen Beispiele, die eine Grundlage für die späteren Bekanntmachungen lieferten, entstammen der Voruntersuchung ,Produktion 2000plus':

- Marktorientierung und strategische Produktplanung

- Technologien und Produktionsausrüstungen

- Neue Formen der Zusammenarbeit produzierender Unternehmen

- Der Mensch und das wandlungsfähige Unternehmen 


\section{Ziele des Rahmenkonzepts}

Mit dem Rahmenkonzept strebt das BMBF insbesondere die folgenden Ziele an:

- die Forschung und Entwicklung auf dem Gebiet der industriellen Produktion zu stärken, wobei neue Entwicklungen auf Gebieten wie Informations- und Kommunikationstechnik, Oberflächen- und Schichttechnologien, Lasertechnik, neue Werkstoffe genutzt werden

- ganzheitliche und nachhaltige - d.h. ökologische, soziale und ökonomische Lösungen für Produktionssysteme zu erforschen und dazu die Zusammenarbeit zwischen Industrie und Forschungsinstituten sowie zwischen verschiedenen Wissenschaftsdisziplinen zu vertiefen

- die breite Anwendung von Forschungsergebnissen in kleinen und mittleren Unternehmen, besonders in den neuen Ländern, zu unterstützen und deren Zusammenarbeit in Unternehmensnetzwerken zu erhöhen

- Anstöße zur Anpassung der beruflichen Aus- und Weiterbildung an zukünftige Anforderungen an die Fachkräfte im Industrieunternehmen zu geben.

\section{Programmsteuerung}

Mit der Abwicklung des Programms betraute das BMBF den Projektträger Produktion und Fertigungstechnologien im Forschungszentrum Karlsruhe (PTKA-PFT). Eine Außenstelle des Projektträgers befindet sich in Dresden.

Erstmals wurde mit dem BMBF Rahmenkonzept „Forschung für die Produktion von morgen“ ein „lernendes Programm“ initiiert, d.h. das Rahmenkonzept hat keine definierte Laufzeit, sondern es soll ständig an das sich kontinuierlich ändernde Umfeld der produzierenden Unternehmen bzw. an die sich ändernden Strategien und internen Abläufe der Unternehmen angepasst werden. Das Rahmenkonzept ist dazu thematisch auch offen konzipiert. Als institutionalisierte Feedback-Schleife hat das BMBF dazu den Gesprächskreis, Strategien für die Produktion im 21. Jahrhundert' eingerichtet. Der Gesprächskreis wird regelmäßig über den Programmverlauf und die vorliegenden Ergebnisse unterrichtet und gibt Empfehlungen für zukünftige Forschungsschwerpunkte des Programms ab. Zusätzlich werden Fachgespräche mit Experten aus Unternehmen und Forschungsinstituten, aus Verbänden und Gewerkschaften durchgeführt, um aktuellen Forschungsbedarf schnell und direkt im Programm aufgreifen zu können.

\section{Bekanntmachungen und Auswahlkriterien}

Die Vergabe von Fördermittel erfolgt auf Grundlage der Ergebnisse von bundesweit bekannt gemachten Ideenwettbewerben. Konsortien von Industrieunternehmen und Forschungseinrichtungen können dabei Projektskizzen zu den in den Bekanntmachungen angeführten Forschungsthemen einreichen. Die beim Projektträger eingegangenen Projektskizzen müssen auf dem internationalen Stand der Technik aufbauen und insbesondere die Ergebnisse und Erkenntnisse vorangegangener Programme und Rahmenkonzepte berücksichtigen. Die Skizzen werden vom Projektträger und einer Gruppe unabhängiger GutachterInnen aus Forschung und Industrie bewertet. Als Kriterien zur Bewertung der Projektskizzen werden herangezogen: 


\section{Zukunftsorientierung}

- Spitzentechnologie, Erreichbarkeit einer Weltspitzenposition

- Neue Fragestellungen und innovative Lösungsansätze

- Risikoreiche Vorhaben

Volkswirtschaftliche Relevanz

- Verbesserung der Wettbewerbsfähigkeit der deutschen Industrie, Stärkung des produzierenden Bereiches in den neuen Ländern

- Erhöhung der Innovationskraft kleiner und mittlerer Unternehmen, Einbindung von jungen Technologiefirmen

- Schaffung und Erhalt von Arbeitsplätzen

- Nachhaltigkeit, ressourcenschonende Produktionsformen, umwelt- und sozialverträgliche Entwicklungen

Systemansatz

- Interdisziplinarität, Übernahme neuer Ergebnisse anderer Wissensgebiete

- Kooperation zwischen Wirtschaft und Wissenschaft

- Konzept zum Projektcontrolling

Breitenwirksamkeit, Aus- und Weiterbildungsaspekte

- Überzeugendes Konzept zur Umsetzung der Ergebnisse

- Einsatzmöglichkeiten für kleine und mittlere Unternehmen aus verschiedenen Wirtschaftszweigen

- Schaffung von Kompetenznetzwerken, Wissenstransfer

- Verknüpfung mit Qualifizierungsstrategien

Zusätzlich können in den einzelnen Bekanntmachungen spezifische Kriterien für die Bewertung der Projektskizzen formuliert werden. Die Partner der Konsortien von positiv bewerteten Projektskizzen werden eingeladen, einen Antrag auf Förderung beim BMBF einzureichen. Unter Berücksichtigung der jeweiligen Haushaltslage werden positiv bewertete Vorhaben finanziell unterstützt.

\section{Vergabe der Fördermittel}

Gefördert werden in der Regel Verbundprojekte. Unter einem Verbundprojekt wird eine vorwettbewerbliche, arbeitsteilige Kooperation von mehreren unabhängigen Partnern aus Wirtschaft und Wissenschaft mit eigenständigen Beiträgen zur Lösung einer Forschungs- und Entwicklungsaufgabe verstanden. Partner können nur gefördert werden, wenn sie in erheblichem Umfang eigene Forschungs- und Entwicklungsarbeiten im Rahmen des Projektes leisten und sonst ihre Ergebnisse hieraus in der vorgesehenen Form Dritten nicht zur Verfügung stellen könnten.

Die maximale möglichen Fördersätze betragen für Unternehmen der gewerblichen Wirtschaft 50\% der Kosten für industrielle Forschung und 25\% für vorwettbewerbliche Entwicklung. Diese Sätze können für KMU und Antragstellern aus den neuen Bundesländern um 10\% erhöht werden, wobei maximal 15\% Erhöhung von KMU aus den neuen Bundesländern kumuliert werden können. Öffentliche Forschungsinstitute (das sind in der Regel Institute der Universitäten und Fachhochschulen 
sowie Fraunhofer-Institute) können mit bis zu 100\% der Kosten gefördert werden. Als Grundregel gilt, dass die gesamten Aufwendungen eines Verbundes maximal zu $50 \%$ gefördert werden können. Da Forschungseinrichtungen in der Regel eine Förderung von $100 \%$ benötigen, wird die Förderquote der Unternehmen entsprechend abgesenkt.

Um eine schnelle Anwendung der Entwicklungen und Projektergebnisse in der industriellen Praxis sicherzustellen, sollen die Forschungsarbeiten hauptsächlich von den beteiligten Unternehmen geleistet, getragen und nachhaltig eingeführt werden. Der Verwertung wie auch der Verallgemeinerung und Verbreitung der erreichten Ergebnisse nach der industriellen Pilotanwendung wird dabei große Bedeutung beigemessen.

Die Fördersumme, die ein Unternehmen erhält, muss in einem vernünftigen Verhältnis zu dem Aufwand stehen, der für die administrative Projektabwicklung notwendig ist. Bei Großunternehmen werden deshalb keine Fördersummen unter 50 Tsd. EUR akzeptiert. Von jedem Unternehmen wird ein Aufwand von möglichst mehr als einem Personenjahr pro Jahr erwartet, wobei überwiegend der Personalaufwand gefördert wird und Sachmittel bei Unternehmen möglichst von diesen selbst getragen werden sollten.

\section{Transfermaßnahmen}

Als wichtigste Maßnahme seitens des Projektträgers zur Unterstützung der Verbreitung von Projektergebnissen aus den geförderten Verbundprojekten auch in den nicht geförderten Bereich unterstützt der Projektträger die Einrichtung so genannter Industriearbeitskreise (IAK). Industriearbeitskreise sollen Wege zur schnellen Verbreitung und Nutzung von Forschungsergebnissen aus Themenfeldern, Verbundprojekten und strategischen Themen bieten.

In einem selbst organisierten Prozess des Informations- und Erfahrungsaustauschs sollen die IAK Teilnehmer die Forschungsergebnisse kennen lernen, sie diskutieren und weiter entwickeln. Sie sollen geförderten und nicht geförderten Unternehmen die Möglichkeit einer längerfristigen Zusammenarbeit bei innovativen Forschungsthemen über Unternehmensgrenzen hinweg bieten. Die Leitung der Industriearbeitskreise obliegt jeweils einem Unternehmen bzw. einer Forschungseinrichtung. Von Seite des Projektträgers wird jeder IAK von jeweils einer Mitarbeiterin / einem Mitarbeiter betreut. Wenn die selbst gestellten Aufgaben als erledigt angesehen werden, löst sich der IAK wieder auf.

\section{Präsentation der Forschungsergebnisse}

Als zentrales Präsentationsforum für die Ergebnisse aus dem BMBF Rahmenkonzept „Forschung für die Produktion von morgen“ veranstaltet der Projektträger alle zwei Jahre die Karlsruher Arbeitsgespräche Produktionsforschung. Neben dem Ideenund Wissensaustausch dienen die Karlsruher Arbeitsgespräche auch der Reflexion des Rahmenkonzepts und dem Suchen nach Inhalten neuer Forschungsfelder. 


\section{$5 \quad$ Kennzahlen zum Rahmenkonzept}

In diesem Abschnitt präsentieren wir die wichtigsten Kennzahlen zur Beteiligung am Rahmenkonzept in den Jahren 1999 bis 2004. Wir skizzieren die Verteilung von Verbundprojekten und Fördermitteln nach den Handlungsfeldern des Programms, sowie nach dem Innovationsgrad der geförderten Vorhaben. Schließlich analysieren wir die Beteiligung am Programm nach verschiedenen Strukturmerkmalen.

\subsection{Beteiligung an den Bekanntmachungen}

In den Jahren 1999 bis 2004 wurden insgesamt zwölf Bekanntmachungen in 22 spezifischen Themenfeldern veröffentlicht. Wissenschaft und Industrie haben sich intensiv am Rahmenkonzept beteiligt. Wie aus Tabelle 1 ersichtlich wurden in den Jahren 1999 bis 2004 mehr als 1.400 Skizzen für Verbundvorhaben beim Projektträger eingereicht. Fast 10.000 Partner haben sich an den Bekanntmachungen beteiligt, wobei rund $73 \%$ der beteiligten Partner in den Skizzen aus der Wirtschaft kamen. Die rege Teilnahme an den Ideenwettbewerben in den Jahren 1999 bis 2004 werten wir als ein wichtiges Indiz, dass mit dem Rahmenkonzept tatsächlich auf Problemlagen in der Produktion Bezug genommen werden konnte.

Stellt man die Anzahl der eingereichten Skizzen den geförderten Verbundvorhaben gegenüber, zeigt sich, dass durchschnittlich jeder ausgewählter Skizze rund acht abgelehnte Skizzen gegenüber standen. Ein FuE-Programm mit einer EinreicherErfolgsquote von ca. 13\% kann sicherlich als selektiv bezeichnet werden. Die hohe Beteiligung hat wahrscheinlich auch damit zu tun, dass in der ersten Stufe des Verfahrens Skizzen ausreichen, jedoch noch keine vollständig ausgearbeiteten Projektpläne eingereicht werden müssen.

Insgesamt ist die hohe Beteiligung an den Bekanntmachungen aber jedenfalls positiv zu vermerken. Es wurde unter den Adressaten des Programms ein hoher Mobilisierungseffekt erzielt, der es im Auswahlverfahren ermöglicht, aus einer Vielzahl von Skizzen die tatsächlich im Sinne der Programmziele innovativsten auszuwählen. Auch bleibt der organisatorische Aufwand bei den Einreichern in der ersten Phase der Projektauswahl in einem vertretbaren Rahmen.

Für eine Beschreibung und Bewertung des administrativen Verfahrens von der Skizze zum abgeschlossenen Projekt verweisen wir auf Abschnitt 7.1 dieses Berichtes. 
Tabelle 1 Übersicht über eingereichte Skizzen zu den Bekanntmachungen in den Jahren 1999 bis 2004

\begin{tabular}{|c|c|c|c|c|c|c|c|c|c|c|c|}
\hline Nr. & $\begin{array}{r}\text { Bekanntmachung } \\
\text { Thema }\end{array}$ & $\begin{array}{r}\text { Anzahl } \\
\text { Skizzen } \\
\end{array}$ & $\begin{array}{c}\text { gepl. Kosten } \\
\text { (Mill.€) }\end{array}$ & $\begin{array}{l}\text { Anzahl } \\
\text { Untern. }\end{array}$ & $\begin{array}{l}\text { davon } \\
\text { KMU }\end{array}$ & $\begin{array}{c}\text { Anzahl } \\
\text { Institute }\end{array}$ & $\begin{array}{r}\text { Anzahl } \\
\text { sonstige }\end{array}$ & $\begin{array}{r}\text { Partner } \\
\text { gesamt }\end{array}$ & $\begin{array}{c}\text { davon } \\
\text { NBL } \\
\end{array}$ & $\begin{array}{l}\text { ausgew. } \\
\text { Skizzen }\end{array}$ & $\begin{array}{l}\text { in } \\
(\%)\end{array}$ \\
\hline 1 & Wachstum / Dienstleistung & 46 & 114 & 298 & 232 & 61 & 46 & 405 & 93 & \multirow{7}{*}{35} & \multirow{7}{*}{$16 \%$} \\
\hline 1 & Mikrotechnik & 41 & 144 & 195 & 108 & 75 & 2 & 272 & 52 & & \\
\hline 1 & Oberflächentechnologie & 34 & 76 & 182 & 98 & 59 & 4 & 245 & 46 & & \\
\hline 1 & Beschäft. Rationalisierung & 20 & 55 & 120 & 48 & 29 & 11 & 160 & 12 & & \\
\hline 1 & Kooperation & 40 & 84 & 262 & 181 & 57 & 51 & 370 & 110 & & \\
\hline 1 & Logistik & 19 & 60 & 100 & 39 & 34 & 1 & 136 & 11 & & \\
\hline 1 & Personalwirtschaft & 13 & 29 & 65 & 26 & 19 & 19 & 103 & 10 & & \\
\hline 2 & Ultraleichtbau & 80 & 87 & 220 & 88 & 62 & 1 & 283 & 35 & 8 & $10 \%$ \\
\hline 3 & Schlüsselkomponenten & 38 & 97 & 153 & 88 & 53 & 4 & 210 & 46 & \multirow{4}{*}{23} & \multirow{4}{*}{$20 \%$} \\
\hline 3 & Kleinserien & 36 & 86 & 228 & 141 & 59 & 6 & 293 & 70 & & \\
\hline 3 & Software in techn. Produkten & 17 & 34 & 90 & 48 & 16 & 0 & 106 & 2 & & \\
\hline 3 & Industrielle Fachkräfte & 22 & 40 & 129 & 64 & 28 & 31 & 188 & 21 & & \\
\hline 4 & Produktentwicklung & 80 & 157 & 420 & 253 & 130 & 28 & 578 & 99 & \multirow{3}{*}{35} & \multirow{3}{*}{$16 \%$} \\
\hline 4 & HL-Fertigungsverfahren & 105 & 209 & 528 & 268 & 150 & 6 & 684 & 104 & & \\
\hline 4 & Flexible, temporäre Fabrik & 32 & 73 & 190 & 109 & 49 & 9 & 248 & 54 & & \\
\hline 5 & Kundenindividuelle Produkte & 33 & 70 & 177 & 139 & 64 & 16 & 257 & 51 & \multirow{3}{*}{22} & \multirow{3}{*}{$14 \%$} \\
\hline 5 & Optische Technologien & 91 & 195 & 431 & 259 & 126 & 11 & 568 & 122 & & \\
\hline 5 & Mikrotechnik & 30 & 100 & 170 & 73 & 44 & 0 & 214 & 14 & & \\
\hline 6 & Optik Design & 43 & 124 & 161 & 95 & 88 & 0 & 249 & 81 & 5 & $12 \%$ \\
\hline 7 & Handhabungstechnik & 96 & 114 & 403 & 270 & 151 & 19 & 573 & 146 & 9 & $9 \%$ \\
\hline 8 & Neue Werkzeugmaschinen & 90 & 279 & 483 & 305 & 137 & 19 & 639 & 96 & 12 & $14 \%$ \\
\hline 9 & Schneller Produktionsanlauf & 138 & 321 & 721 & 447 & 223 & 34 & 978 & 198 & 5 & $4 \%$ \\
\hline 10 & Produktionsketten Mikroteile & 79 & 225 & 422 & 309 & 111 & 6 & 539 & 116 & 6 & $8 \%$ \\
\hline 10 & Integrierte Modernisierung & 80 & 191 & 515 & 363 & 140 & 77 & 732 & 139 & 6 & $8 \%$ \\
\hline 11 & Nanomobil & 1 & 2 & 3 & 1 & 1 & 0 & 4 & 0 & 1 & - \\
\hline 12 & Montage & 39 & 89 & 193 & 130 & 59 & 10 & 262 & 46 & 8 & $18 \%$ \\
\hline 12 & Fügen & 78 & 171 & 420 & 240 & 120 & 13 & 553 & 94 & 10 & $13 \%$ \\
\hline & Gesamt (1999 bis 2004) & 1.421 & 3.226 & 7.279 & 4.422 & 2.145 & 424 & 9.849 & 1.868 & 185 & $13 \%$ \\
\hline
\end{tabular}

Quelle: Zusammenstellung aus Präsentationsunterlagen des BMBF und des Projektträgers PTKA-PFT 


\subsection{Programmumsetzung nach Handlungsfeldern}

Im Rahmenkonzept „Forschung für die Produktion von morgen“ wurden von 1999 bis Ende 2004 insgesamt 166 Verbundprojekte (bereits abgeschlossene und laufende Projekte) unterstützt, für die 264,1 Mio. EUR Fördermittel zur Verfügung gestellt wurden. Pro Jahr wurden damit etwa 53 Mio. EUR gebunden. ${ }^{1}$ Im Durchschnitt arbeiteten sieben verschiedene Industriebetriebe und Institute in einem Verbundvorhaben zusammen. Die Spannweite der Teilnehmerzahl ist durchaus beträchtlich und reicht von zwei bis 20 Partnern in einem Verbundvorhaben. Je Verbund werden im Mittel etwa 1,6 Mio. EUR Fördermittel bereitgestellt, wobei auch hier eine beträchtliche Spannweite von 255 Tsd. EUR bis ca. 4,2 Mio. EUR existiert.

Tabelle 2 gibt uns Anhaltspunkte dafür, dass die Bedeutung des Handlungsfelds 2: Technologien und Produktionsausrüstungen im Beobachtungszeitraum kontinuierlich zugenommen hat. Während unter den 1999 und 2000 gestarteten Vorhaben weniger als jedes dritte Verbundprojekt dem Handlungsfeld 2 zugeordnet wurde, lag der Anteil bei den in den Jahren 2001 und 2002 gestarteten Projekten bereits bei mehr als der Hälfte der Vorhaben und in den Jahren 2003 und 2004 bei 80\%.

\section{Tabelle 2 Verbundprojekte nach Handlungsfeld und Jahr}

\begin{tabular}{|c|c|c|c|c|c|c|c|c|}
\hline \multirow[b]{2}{*}{ Handlungsfeld } & \multicolumn{8}{|c|}{ Anzahl Verbundprojekte mit Projektbeginn in Jahr ... } \\
\hline & 1999 & 2000 & 2001 & 2002 & 2003 & 2004 & Gesamt & in $\%$ \\
\hline Marktorientierung und strategische & & & & & & & & \\
\hline Produktplanung & & 6 & 23 & 8 & 1 & & 38 & 23 \\
\hline Technologien und Produktionsausrüstungen & & 10 & 36 & 18 & 22 & 6 & 92 & 55 \\
\hline $\begin{array}{l}\text { Neue Formen der Zusammenarbeit } \\
\text { produzierender Unternehmen }\end{array}$ & & 8 & 3 & 3 & & 1 & 15 & 9 \\
\hline Der Mensch und das wandlungsfähige & & & & & & & & \\
\hline Unternehmen & 2 & 8 & 4 & 2 & & 5 & 21 & 13 \\
\hline Gesamt & 2 & 32 & 66 & 31 & 23 & 12 & 166 & 100 \\
\hline
\end{tabular}

Quelle: PROFI Datenbank, Projektträger PTKA-PFT

Auch wenn wir die Verteilung der Fördermittel nach Handlungsfeldern betrachten, zeigt sich, dass der Schwerpunkt der Projektförderung in den Jahren 1999 bis 2004 eindeutig auf den Bereich der Fertigungstechnologie gelegt wurde, auf den das Handlungsfeld 2 zielt. Für Themen, die nicht unmittelbar die Entwicklung innovativer Produktionsverfahren und Produktionsausrüstung verfolgen, wurden rund $31 \%$ der Fördermittel im Rahmenkonzept bereitgestellt.

Die durchschnittliche Projektgröße der Verbundvorhaben variiert von Handlungsfeld zu Handlungsfeld etwas, aber nicht sehr stark. Mit durchschnittlich 1,64 Mio. EUR sind die Verbundvorhaben im Handlungsfeld 2 etwas umfangreicher dimensioniert als die Verbundvorhaben im Handlungsfeld 3, auf die mit durchschnittlich 1,31 Mio. EUR im Mittel die geringsten Fördermittel entfallen. In Handlungsfeld 3 ist auch die Spannweite der Projektgrößen vergleichsweise am geringsten; sie reicht von etwa 440 Tsd. EUR bis etwa 3 Mio. EUR. Die größte Spannweite der Projektgrößen hinsichtlich der bewilligten Fördermittel zeigt sich im Handlungsfeld 2, wo Projekte von etwa 620 Tsd. EUR bis etwa 4,2 Mio. EUR gefördert werden. Die Handlungs-

1 Die geförderten Verbundprojekte können nicht vollständig den zwölf Bekanntmachungen zugeordnet werden. Frühe Projekte haben ihren Ursprung noch im Vorläuferprogramm. Ausgewählte Skizzen aus dem Jahr 2004 starteten teilweise erst im Jahr 2005. 
felder 1 und 4 liegen mit mittleren Projektgrößen (Fördervolumen) von 1,56 Mio. EUR bzw. 1,47 Mio. EUR zwischen den beiden anderen Handlungsfeldern.

\section{Abbildung 3 Verteilung der Fördermittel nach Handlungsfeldern}

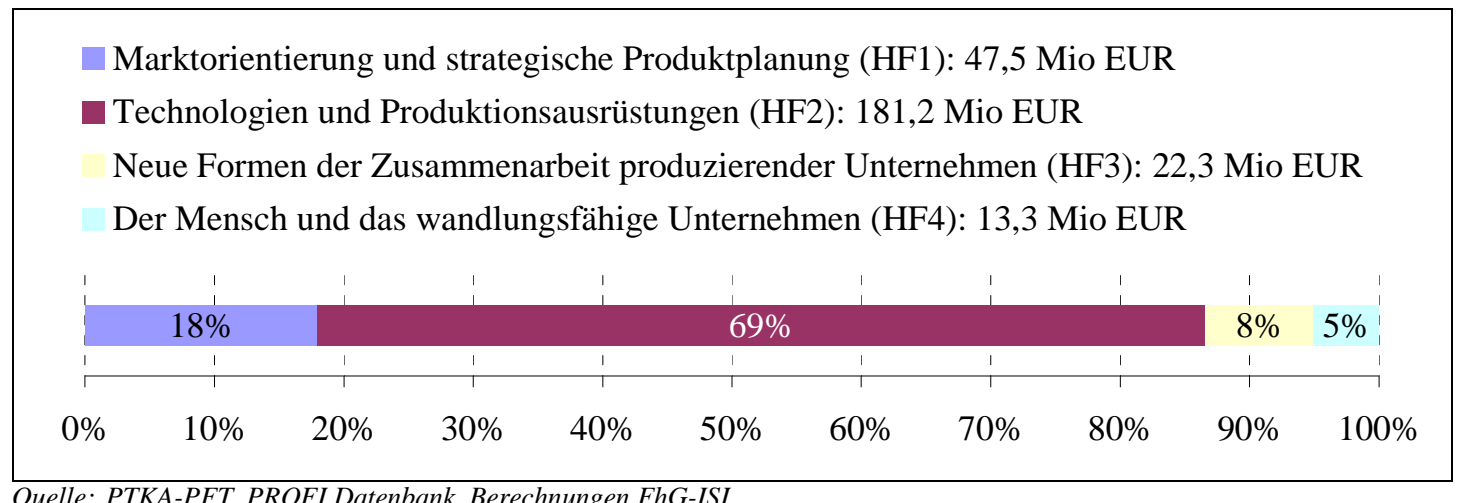

Ein weiterer Anhaltspunkt, dass über den Betrachtungszeitraum die Bedeutung technischer Verbundprojekte zugenommen hat, ergibt sich, wenn wir die Verbundprojekte danach kategorisieren, welche Dimension von Innovation (d.h. technologieorientiert, organisationsorientiert bzw. strategieorientiert) überwiegend adressiert wird (siehe Tabelle 3). Dabei zeigt sich, dass von den zwischen Programmbeginn und Ende 2004 gestarteten Verbundprojekten mehr als zwei Drittel technologieorientierte Innovationen verfolgten (68\%). Jedes fünfte Verbundprojekt (20\%) widmete sich organisationsorientierten FuE-Aufgaben (z.B. neue Kooperationsformen) und etwa jedes achte Verbundprojekt (12\%) beschäftigte sich hauptsächlich mit strategisch orientierten FuE-Vorhaben (z.B. neue Geschäftsmodelle). Über den Evaluationszeitraum betrachtet zeigt sich also, dass technologieorientierte Verbundprojekte zunehmend an Bedeutung gewonnen haben und insbesondere strategieorientierte Themen in immer geringerem Umfang Gegenstand von Verbundprojekten waren.

Tabelle 3 Verbundprojekte nach Innovationsorientierung und Jahr

\begin{tabular}{lrrrrrrrr}
\hline & \multicolumn{7}{c}{ Anzahl Verbundprojekte mit Projektbeginn } \\
Innovationstyp & 1999 & 2000 & 2001 & 2002 & 2003 & 2004 & Gesamt & in \% \\
\hline technologieorientiert & & 11 & 52 & 21 & 22 & 7 & 113 & 68 \\
organisationsorientiert & 2 & 11 & 8 & 6 & 1 & 5 & 33 & 20 \\
strategieorientiert & & 10 & 6 & 4 & & & 20 & 12 \\
\hline Gesamt & 2 & 32 & 66 & 31 & 23 & 12 & 166 & 100 \\
\hline Quelle: PROFI Datenbank, Projektträger PTKA-PFT & & & & & & & &
\end{tabular}

Es stellt sich die Frage, ob der Anteil von $8 \%$ der Fördermittel für den Themenbereich Unternehmenskooperationen und -netzwerke (Handlungsfeld 3: Neue Formen der Zusammenarbeit produzierender Unternehmen) und von etwa $5 \%$ für den Themenbereich Personal und Wandlungsfähigkeit (Handlungsfeld 4: Der Mensch und das wandlungsfähige Unternehmen ausreichen), um diese explizit und prominent im Rahmenkonzepts angesprochenen Themen adäquat aufgreifen $\mathrm{zu}$ können. Jedenfalls gibt es aus Sicht der Evaluation keinen Befund mit dem eine Abwendung vom integrativen Ansatz des Rahmenkonzepts begründet werden könnte. 


\subsection{Angestrebte Innovationen in den geförderten FuE-Vorhaben}

Hinweise über die angestrebten Innovationen in den geförderten FuE-Vorhaben geben uns die Ergebnisse der Teilnehmerbefragung. Wir fragten die Programmteilnehmer nach dem verfolgten inhaltlichen Projektziel, wobei wir vier Antwortkategorien vorgaben, die unterschiedliche Ebenen von Innovation widerspiegeln. In Abbildung 4 sind die Antworten der Befragten zusammengefasst.

Für die beteiligten Industriepartner halten sich Neuentwicklungsinnovationen (41\%) und Verbesserungsinnovationen (40\%) als inhaltliches Projektziel die Waage. Allgemeiner Erfahrungsaustausch und Kompetenzaufbau bzw. Grundlagenentwicklung spielt bei den Industriebetrieben eine vergleichsweise geringe Rolle. Bei den Institutspartnern dominiert als Ziel eindeutig die Entwicklung neuer Produkte bzw. bzw. neuer Verfahren, Dienstleistungen, Geschäftsmodelle, organisatorischer Abläufe etc. (52\%). Verbesserungsinnovationen sind für diese Gruppe als Entwicklungsziel von geringerer Bedeutung. Gleichzeitig spielt wenig überraschend die Grundlagenentwicklung für Institutspartner eine größere Rolle.

\section{Abbildung 4 Angestrebte Innovationen in den geförderten FuE-Vorhaben}

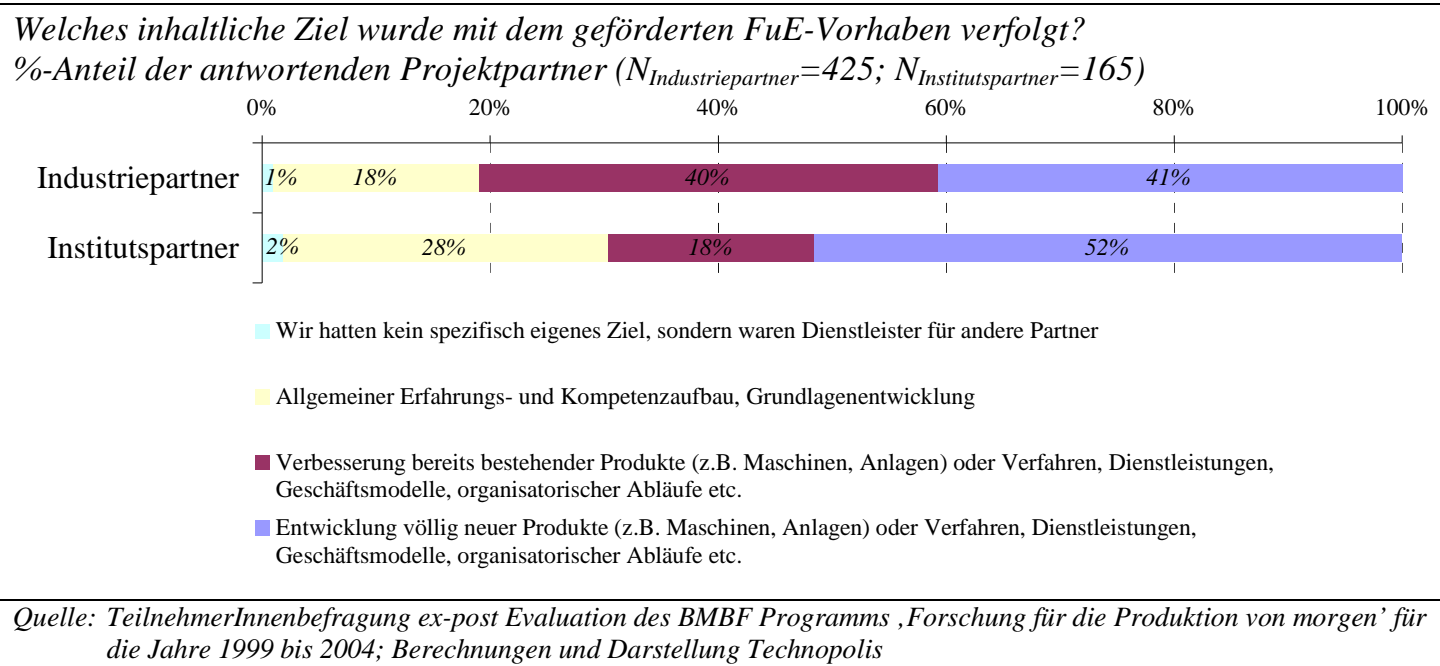

Zwischen FuE-Vorhaben in den unterschiedlichen Handlungsfeldern des Programms lassen sich keine statistisch signifikanten Unterschiede hinsichtlich des angestrebten Innovationen der FuE-Vorhaben feststellen. Die Verteilung der FuE-Vorhaben in mehr ambitionierte (im Sinne von Neuentwicklungen) und weniger ambitionierte Projekte (im Sinne von Verbesserungen bereits bestehender Lösungen) entlang der Antwortkategorien ist über alle vier Handlungsfeldern hinweg vergleichbar.

Es stellte sich für uns die Fragte, ob der doch beträchtliche Anteil an FuE-Vorhaben die Verbesserungsinnovationen anstreben, tatsächlich im Einklang mit dem Programmanspruch steht, oder ob nicht mehr Augenmerk auf anspruchsvollere Projekte, die auf Neuentwicklungen abzielen, gelegt werden sollte. Wir verstehen darunter keinesfalls nur technisch-orientierte Projekte. Die Ergebnisse der Teilnehmerbefragung zu den Projektwirkungen in den Betrieben (siehe Abschnitt 6.3) legt nahe, dass FuE-Vorhaben zu neuen technischen, organisatorischen und strategischen Lösungen besonders erfolgreiche Ergebnisse nach sich ziehen. Gleichzeitig sind wir uns bewusst, dass es in der Produktionsforschung nicht nur darum gehen kann, neue Dinge zu tun, sondern Dinge besser zu tun. 


\subsection{Analyse der Teilnehmerstruktur}

In diesem Abschnitt beschreiben wir die am Programm teilnehmende Klientel entlang der Branchen- und Größenstruktur der teilnehmenden Industriebetriebe sowie nach ihrer regionalen Herkunft und Rolle im Verbundprojekt. Dabei geht es uns darum zu erfahren, in wie weit die Zielgruppe des Rahmenkonzepts erreicht werden konnte. Für eine ausführliche Analyse der Teilnehmer am Programm verweisen wir auf den Bericht zum Arbeitspaket 2: Klientelanalyse (siehe Appendix A).

Insgesamt waren in den 166 Verbundvorhaben, die in den Jahren 1999 bis 2004 starteten in Summe 1.157 Projektpartner beteiligt, davon 894 Industriebetriebe $(77,3 \%)$ und 263 Institute $(22,7 \%)$. Bereinigt man diese Teilnehmerzahlen um die Anzahl der Mehrfachteilnehmer, so reduziert sich die Gesamtzahl der erreichten Industriebetriebe und Institute auf insgesamt 848. Die 704 Industriebetriebe nehmen in dieser um Mehrfachteilnehmer bereinigten Betrachtung mit $83 \%$ gegenüber den 144 Forschungsinstituten mit $17 \%$ weiter zu. Dies zeigt, dass Institute im Rahmenkonzept deutlich häufiger mehrfach gefördert werden (45\%) als Industriebetriebe, die zu $21 \%$ mehr als einmal in Verbundvorhaben mitwirken. Von den Fördermitteln entfällt mit 166 Mio. EUR oder knapp $63 \%$ der Großteil auf die an den Verbundvorhaben beteiligten Industriebetriebe. Der Institutsanteil beträgt etwa 98 Mio. EUR, das sind etwa $37 \%$ der gesamte Fördermittel.

\section{Beteiligung am Rahmenkonzept 1999 bis 2004 nach Wirtschaftsklassen}

Differenzieren wir die Industriepartner der geförderten Verbundvorhaben nach Branchen, so zeigt sich folgendes Bild (siehe Tabelle 4): Mit ca. $26 \%$ der beteiligten Industriebetriebe stellt der Maschinenbau die bedeutendste Branche in den geförderten Verbundvorhaben dar. Davon sind etwa $11 \%$ der Betriebe dem sonstigen Maschinenbau zuzurechnen. Mit über $7 \%$ Beteiligten sind Hersteller von Werkzeugmaschinen die zweitstärkste Teilgruppe des Maschinenbaus, die in Verbundvorhaben vertreten ist. Insbesondere diese Gruppe sowie mit Abstrichen auch die Hersteller von Maschinen für die Erzeugung und Nutzung mechanischer Energie sind, bezogen auf ihren Anteil an den beteiligten Betrieben des Verarbeitenden Gewerbes, gegenüber der Grundgesamtheit deutlich stärker repräsentiert.

An zweiter Stelle der beteiligten Betriebe folgen mit knapp $17 \%$ die Hersteller von Geräten der Medizin-, Mess-, Steuerungs-, Regelungstechnik und Optik. Von diesen entfällt mit knapp $10 \%$ der größte Anteil auf die Hersteller von Messgeräten etc. Auch die Hersteller von optischen Geräten sowie die Hersteller von Steuerungen, die jeweils etwas mehr als $3 \%$ der teilnehmenden Betriebe repräsentieren, sind gegenüber der Grundgesamtheit des Verarbeitenden Gewerbes überdurchschnittlich im Programm vertreten. Dagegen sind die Hersteller von medizintechnischen Geräten im Vergleich zur Grundgesamtheit des Verarbeitenden Gewerbes eher unterrepräsentiert.

Die drittstärkste Branche der Teilnehmer an den Verbundhaben des Rahmenkonzepts sind die Hersteller des Fahrzeugbaus (WZ 34 und 35). Auf diese Branche entfallen mit ca. 8,7 \% deutlich mehr Betriebe als ihr Anteil in der Grundgesamtheit des Verarbeitenden Gewerbes beträgt. 
Die viertstärkste Gruppe der an den Verbundvorhaben des Rahmenkonzepts teilnehmenden Betriebe stellen die Hersteller von Metallerzeugnissen mit 8,4\% der Teilnehmer dar. Diese Gruppe ist damit insgesamt geringer vertreten als in der Grundgesamtheit des Verarbeitenden Gewerbes. Die Teilgruppe der Werkzeughersteller jedoch ist mit etwa 2,6 \% der Teilnehmer stärker repräsentiert als dies in der Grundgesamtheit des Verarbeitenden Gewerbes der Fall ist. Betriebe der ehemaligen Elektrotechnik-Branchen (WZ 30 bis 32) stellen etwa 7,7 \% der an Verbundvorhaben beteiligten Betriebe. Damit sind sie etwas stärker in den Verbundvorhaben des Rahmenkonzeptes vertreten als es ihrer Bedeutung hinsichtlich des Betriebsanteils am gesamten Verarbeitenden Gewerbe entspricht.

\section{Tabelle 4 Struktur der teilnehmenden Betriebe nach Branchen}

\begin{tabular}{|c|c|c|c|c|}
\hline & \multirow{2}{*}{$\begin{array}{l}\text { Branche } \\
\text { WZ }\end{array}$} & \multicolumn{2}{|c|}{$\begin{array}{l}\text { Geförderte } \\
\text { Betriebe }\end{array}$} & \multirow{2}{*}{$\begin{array}{c}\text { Fördermittel- } \\
\text { anteil } \\
\%\end{array}$} \\
\hline & & $\mathrm{N}$ & $\%$ & \\
\hline $\begin{array}{l}\text { Verlag/Druck, Mineralöl, Ernährungsgewerbe, Textil-, } \\
\text { Bekleidungs-, Leder- und Holzgewerbe (Low-Tech) }\end{array}$ & $\begin{array}{l}15,17,18,19 \\
20,22,23\end{array}$ & 20 & 2,2 & 1,4 \\
\hline Chemische Industrie & 24 & 16 & 1,8 & 1,4 \\
\hline Herstellung von Gummi- und Kunststoffwaren & 25 & 19 & 2,1 & 2,0 \\
\hline Glasgew., Keramik, Verarbeitung v. Steinen u. Erden & 26 & 17 & 1,9 & 2,0 \\
\hline Metallerzeugung und -bearbeitung & 27 & 30 & 3,4 & 2,7 \\
\hline Herstellung von Metallerzeugnissen & 28 & 75 & 8,4 & 7,9 \\
\hline H. v. Metallerzeugnissen (ohne Werkzeuge) & 28 (ohne 28.6) & 52 & 5,8 & 6,0 \\
\hline H. v. Werkzeugen etc. & 28.6 & 23 & 2,6 & 1,9 \\
\hline Maschinenbau & 29 & 231 & 25,9 & 26,7 \\
\hline $\begin{array}{l}\text { H. v. Maschinen f. d. Erzeugung u. Nutzung } \\
\text { mechanischer Energie }\end{array}$ & 29.1 & 58 & 6,5 & 6,5 \\
\hline H. v. land- u. forstw. Maschinen & 29.3 & 6 & 0,7 & 0,6 \\
\hline H.v. Werkzeugmaschinen & 29.4 & 65 & 7,3 & 7,5 \\
\hline sonst. Maschinenbau & 29 (ohne o.g.) & 102 & 11,4 & 12,1 \\
\hline $\begin{array}{l}\text { H. v. Büromasch., Datenverarbeitungsgeräten u. -ein- } \\
\text { richtungen, Geräten der Elektrizitätserz., -verteilung u. } \\
\text { ä. u. Rundfunk-, Fernseh- u. Nachrichtentechnik }\end{array}$ & $30,31,32$ & 68 & 7,7 & 8,4 \\
\hline Medizin-, Mess-, Steuer- und Regelungstechnik, Optik & 33 & 149 & 16,6 & 18,7 \\
\hline H. v. med. Geräten u. orthopäd. Vorrichtungen & 33.1 & 3 & 0,3 & 0,2 \\
\hline H. v. Mess-, Kontroll-, Navig.- u.ä. Instr. u. Vorr. & 33.2 & 87 & 9,7 & 11,2 \\
\hline H. v. industriellen Prozess-, Steuerungsanlagen & 33.3 & 29 & 3,2 & 3,4 \\
\hline H. v. optischen u. fotografischen Geräten und Uhren & 33 (ohne o.g.) & 30 & 3,4 & 3,9 \\
\hline Fahrzeugbau & 34,35 & 78 & 8,7 & 7,4 \\
\hline $\begin{array}{l}\text { Herstellung v. Möbeln, Schmuck, Musikinstrumenten, } \\
\text { Sportgeräten, Spielwaren und sonstigen Erzeugnissen }\end{array}$ & 36 & 10 & 1,1 & 0,7 \\
\hline Verarbeitendes Gewerbe (gesamt) & D & 713 & 79,8 & 79,3 \\
\hline Datenverarbeitung und Datenbanken & 72 & 76 & 8,5 & 10,5 \\
\hline Forschung und Entwicklung & 73 & 35 & 3,9 & 4,7 \\
\hline Erbringung von Dienstleistungen $\mathrm{f}$. Unternehmen & 74 & 47 & 5,3 & 3,8 \\
\hline Sonstige & $\begin{array}{l}10,40,45,51, \\
63,64,65,70\end{array}$ & 23 & 2,6 & 1,7 \\
\hline Gesamt & & 894 & 100 & 100 \\
\hline
\end{tabular}

Betriebe der Prozessindustrie (Chemische Industrie, Gummi- und Kunststoffwaren, Glas und Keramik) und der Low-Tech Branchen (Verlag/Druck, Mineralöl, Ernährungsgewerbe, Textil-, Bekleidungs-, Leder- und Holzgewerbe) sind im Vergleich zur Grundgesamtheit des Verarbeitenden Gewerbes unterdurchschnittlich in den 
Verbundvorhaben repräsentiert. Insgesamt setzt das Rahmenkonzept also vorrangig auf die Stärkung der in Deutschland traditionell starken Stückgut fertigenden

Betriebe aus dem Bereich der höherwertigen Technologie.

\section{Tabelle 5 Struktur der teilnehmenden Betriebe aus dem Verarbeitenden Gewerbe im Vergleich zur Grundgesamtheit}

\begin{tabular}{|c|c|c|c|c|c|}
\hline & \multirow{2}{*}{$\begin{array}{l}\text { Branche } \\
\text { WZ }\end{array}$} & \multicolumn{2}{|c|}{ Grundgesamtheit } & \multicolumn{2}{|c|}{$\begin{array}{l}\text { Geförderte } \\
\text { Betriebe }\end{array}$} \\
\hline & & $\mathrm{N}$ & $\%$ & $\mathrm{~N}$ & $\%$ \\
\hline $\begin{array}{l}\text { Verlag/Druck, Mineralöl, Ernährungsgewerbe, Textil-, } \\
\text { Bekleidungs-, Leder- und Holzgewerbe (Low-Tech) }\end{array}$ & $\begin{array}{l}15,17,18,19 \\
20,22,23\end{array}$ & 34.267 & 34,7 & 20 & 2,8 \\
\hline Chemische Industrie & 24 & 3.250 & 3,3 & 16 & 2,2 \\
\hline Herstellung von Gummi- und Kunststoffwaren & 25 & 6.131 & 6,2 & 19 & 2,7 \\
\hline Glasgewerbe, Keramik, Verarb. v. Steinen u. Erden & 26 & 6.043 & 6,1 & 17 & 2,4 \\
\hline Metallerzeugung und -bearbeitung & 27 & 1.665 & 1,7 & 30 & 4,2 \\
\hline Herstellung von Metallerzeugnissen & 28 & 16.243 & 16,5 & 75 & 10,5 \\
\hline H. v. Metallerzeugnissen (ohne Werkzeuge) & 28 (ohne 28.6) & 14.168 & 14,4 & 52 & 7,3 \\
\hline H. v. Werkzeugen etc. & 28.6 & 2.075 & 2,1 & 23 & 3,2 \\
\hline Maschinenbau & 29 & 11.036 & 11,3 & 231 & 32,3 \\
\hline $\begin{array}{l}\text { H. v. Masch.f.d. Erzeugung u. Nutzung mech. } \\
\text { Energie }\end{array}$ & 29.1 & 1.829 & 1,9 & 58 & 8,1 \\
\hline H. v. land- u. forstw. Maschinen & 29.3 & 287 & 0,3 & 6 & 0,8 \\
\hline H. v. Werkzeugmaschinen & 29.4 & 1.436 & 1,5 & 65 & 9,1 \\
\hline sonst. Maschinenbau & 29 (ohne o.g.) & 7.484 & 7,6 & 102 & 14,3 \\
\hline $\begin{array}{l}\text { H. v. Büromaschinen, Datenverarbeitungsgeräten u. } \\
\text {-einrichtungen, Geräten der Elektrizitätserz., -vert. u. } \\
\text { ä. u. Rundfunk-, Fernseh- und Nachrichtentechnik }\end{array}$ & $30,31,32$ & 6.483 & 6,6 & 68 & 9,5 \\
\hline Medizin-, Mess-, Steuer- u. Regelungstechnik, Optik & 33 & 4.170 & 4,1 & 149 & 20,9 \\
\hline H. v. med. Geräten u. orthopäd. Vorrichtungen & 33.1 & 1.612 & 1,6 & 3 & 0,4 \\
\hline H. v. Mess-, Kontroll-, Navig.- u.ä. Instr. u. Vorr. & 33.2 & 1.825 & 1,8 & 87 & 12,2 \\
\hline H. v. industriellen Prozess-, Steuerungsanlagen & 33.3 & 204 & 0,2 & 29 & 4,1 \\
\hline H. v. optischen u. fotografischen Geräten u. Uhren & 33 (ohne o.g.) & 529 & 0,5 & 30 & 4,2 \\
\hline Fahrzeugbau & 34,35 & 2.073 & 2,1 & 78 & 10,9 \\
\hline $\begin{array}{l}\text { Herst. v. Möbeln, Schmuck, Musikinstrumenten, } \\
\text { Sportgeräten, Spielwaren und sonst. Erzeugnissen }\end{array}$ & 36 & 4.803 & 4,9 & 10 & 1,4 \\
\hline Sonst. Branchen des Verarbeitenden Gewerbes & $16,21,37$ & & 2,6 & - & - \\
\hline Verarbeitendes Gewerbe (gesamt) & & 98.725 & 100 & 713 & 100 \\
\hline
\end{tabular}

Neben Betrieben des Verarbeitenden Gewerbes sind in den Verbundvorhaben des

Rahmenkonzepts auch Betriebe aus spezifischen Dienstleistungssektoren mit signifikanten Anteilen vertreten. So stellen die Bereiche Datenverarbeitung und Datenbanken (8,5 \%), Forschung und Entwicklung (3,9\%) sowie Erbringung von Dienstleistungen überwiegend für Unternehmen (5,3\%) insgesamt knapp $18 \%$ der im Rahmenkonzept beteiligten Betriebe. Dies zeigt, dass zur Entwicklung innovativer Lösungen im Bereich der Fertigungstechnologie und Planungsmethoden für Fertigungsprozesse auch Spezialkompetenzen aus diesen wissensintensiven produktionsnahen Dienstleistungsbereichen benötigt werden.

Analysiert man die Beteiligung der geförderten Betriebe nach Branchen nicht auf Basis der Anzahl der teilnehmenden Betriebe, sondern anhand der auf die jeweiligen Branchen entfallenden Fördersummen, so zeigt sich ein kaum verändertes Bild (siehe Tabelle 5). Die Low-Tech-Sektoren sowie die Betriebe der Prozessindustrie sind noch etwas stärker unterproportional repräsentiert, während die Schwerpunkt- 
setzung auf Betriebe des Maschinenbaus und der Medizin-, Mess-, Steuer- und Regelungstechnik sowie Optik noch (leicht) stärker hervortritt. Auffällig ist, dass die Hersteller von Fahrzeugen und Fahrzeugteilen beim Anteil an der Förderung im Vergleich zur Anzahl der beteiligten Betriebe mit nunmehr 7,4 \% etwas an Bedeutung verlieren. Dies könnte darauf hin deuten, dass insbesondere Betriebe dieser Branche als Pilotanwender bzw. Lead User, für deren industriellen Einsatz bestimmte Fertigungstechnologien und Methoden entwickelt werden, mit im Mittel geringeren Anwenderanteilen in die Verbundvorhaben integriert werden. Dieser Frage soll im weiteren Verlauf nachgegangen werden, wenn wir die Beteiligung der Industriebetriebe und Institute nach den Rollen, die sie in den Verbundvorhaben einnehmen, analysieren werden.

Bemerkenswert ist, dass die Betriebe der wissensintensiven produktionsnahen Dienstleistungssektoren (Datenverarbeitung und Datenbanken, Forschung und Entwicklung, Dienstleistung überwiegend für Unternehmen) gemessen am Anteil der Fördersumme eine noch leicht höhere Bedeutung haben als gemessen an der Anzahl der beteiligten Betriebe. Diese Betriebe scheinen also nicht nur mit kleinen Entwicklungsaufgaben für periphere Lösungen beteiligt zu werden, sondern durchaus Spezialkompetenzen einzubringen, die für den Projekterfolg wesentlich sein können.

Insgesamt erreichte die Förderung im Rahmenkonzept mit 894 beteiligten Industriebetrieben etwa 0,9\% aller Betriebe des Verarbeitenden Gewerbes, bereinigt um Mehrfachteilnahmen noch 704 Betriebe, entsprechend etwa 0,7 \%. Da die bis Ende 2004 für Industriepartner bewilligten Fördermittel in Höhe von 166 Mio. EUR entsprechend etwa 33 Mio. EUR pro Jahresscheibe (2000 bis 2004) nur etwa 0,08\% der jährlichen FuE-Aufwendungen des Verarbeitenden Gewerbes ausmachen, ist die erreichte Betriebsbeteiligung durchaus beachtlich. Damit wird auch deutlich, dass der Verbundprojektansatz des Rahmenkonzepts nicht daran gemessen werden kann, einen Großteil der potenziellen industriellen Zielgruppe direkt zu erreichen.

\section{Größenstruktur der industriellen Projektteilnehmer}

Wenn wir die Größenverteilung der im Rahmenkonzept „Forschung für die Produktion von morgen“" geförderten Industriebetriebe analysieren, so zeigt sich folgendes Bild (siehe Abbildung 5): Betriebe mit weniger als 100 Beschäftigten stellen mit insgesamt $44 \%$ der projektbeteiligten Industriebetriebe die zahlenmäßig bedeutendste Gruppe. Weitere $16 \%$ der beteiligten Industriebetriebe zählen 100 bis 250 Beschäftigte. Von den in den Verbundvorhaben des Rahmenkonzepts beteiligten Industriebetrieben sind $60 \%$ kleine und mittlere Unternehmen (KMU) nach EU Definition $^{2}$. Auf die Gruppe der Industriebetriebe mit über 250 bis 500 MitarbeiterInnen entfallen weitere $9 \%$ der Beteiligten. Insgesamt $31 \%$ der beteiligten Industriebetriebe zählen 500 und mehr MitarbeiterInnen.

2 Diese Quote ist nicht deckungsgleich mit dem Anteil der Industriebetriebe, die aufgrund der Einträge in die Profi-Datenbank als KMU bzw. KMU im Verbund eines Großunternehmens ausgewiesen waren, da diese Einklassifizierung nicht streng der EU KMU-Definition folgt. 
Abbildung 5 Größenstruktur der beteiligten Industriepartner

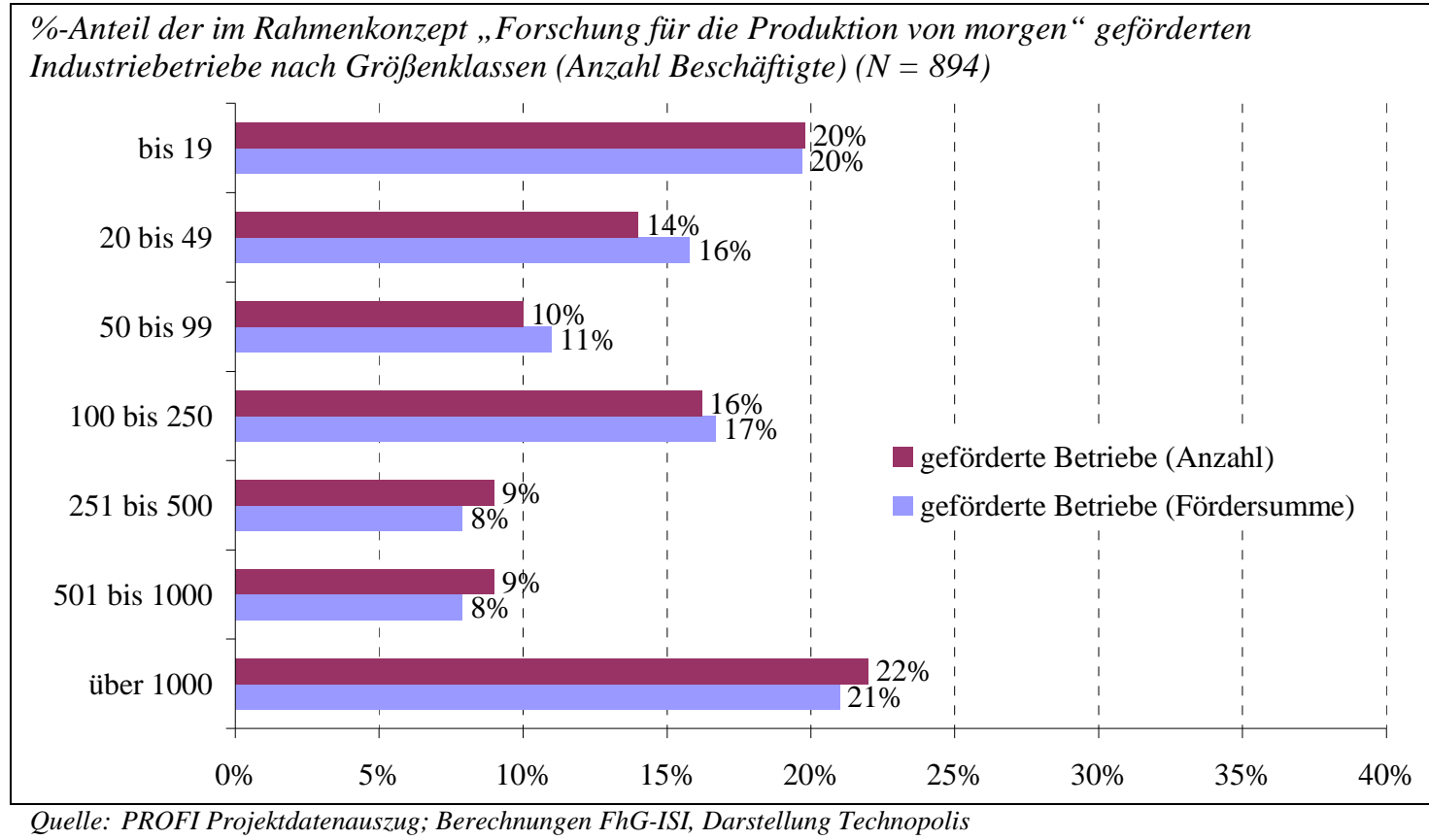

Analysieren wir die Beteiligung von Industriebetrieben unterschiedlicher Größe nicht auf Basis der Anzahl geförderter Unternehmen, sondern anhand der bewilligten Anteile an den Fördermitteln, so zeigt sich ein nur marginal verändertes Bild. Von den insgesamt 166 Mio. EUR, die als Förderung der Gruppe der Industriebetriebe zugute kommen (63 \% im Vergleich zu $37 \%$ Förderung für Forschungsinstitute), entfielen insgesamt $47 \%$ auf Industriebetriebe mit weniger als 100 Beschäftigten. $\mathrm{Zu}$ weiteren $17 \%$ partizipieren Industriebetriebe mit 100 bis 250 Beschäftigten an der Förderung. Damit kommen kleinen und mittleren Unternehmen (KMU) etwa $64 \%$ der für die Industrie bereitgestellten Fördermittel zugute. Die weiteren Fördermittel für Industriebetriebe entfielen zu $8 \%$ auf Betriebe mit 250 bis 500 Beschäftigten und zu $29 \%$ auf Betriebe mit mehr als 500 Beschäftigten.

Die im Vergleich zur Anzahl der Betriebe höhere KMU-Zuteilungsquote weist darauf hin, dass entgegen einer möglichen Vermutung, wonach die Projekte von KMU im Vergleich zu den Projekten von Großunternehmen eher unterdurchschnittlich ausgestattet sein könnten, die Projekte bei KMU im Mittel gerade etwas größer sind. Eine mögliche Erklärung für diesen Sachverhalt könnte sein, dass große Unternehmen teilweise als Pilotanwender mit relativ geringem Volumen in die Entwicklungsvorhaben des Rahmenkonzepts miteingebunden wurden.

Der errechnete Anteil von ca. $64 \%$ des Fördervolumens für KMU mit weniger als 250 MitarbeiterInnen deckt sich nicht völlig mit der in der vom BMBF herausgegebenen Broschüre „Von der Projektidee in die Produktion“ ausgewiesenen KMU Quote von $70 \%$ der Fördermittel für die Industrie. Dies liegt u. a. daran, dass in der Vergangenheit in der vom BMBF bzw. im PROFI System gewählten Abgrenzung nicht die KMU EU-Definition zugrunde gelegt wird, sondern einer Mittelstandsdefinition folgte, die als Umsatzgrenze 100 Mio. EUR vorsah.

Will man die Beteiligungsquoten nach Größenklassen der geförderten Betriebe mit der Grundgesamtheit vergleichen, so ist dies nur für die 713 geförderten Betriebe des 
Verarbeitenden Gewerbes sinnvoll, da der Dienstleistungssektor aufgrund seiner Heterogenität keine Aussagen zulässt. Die Betriebe des Verarbeitenden Gewerbes stellen knapp $80 \%$ der geförderten Industriebetriebe. Der auf dem Verarbeitenden Gewerbe beruhende Vergleich mit der Grundgesamtheit zeigt, dass an den geförderten Verbundvorhaben überproportional größere Betriebe teilnehmen (siehe Abbildung 6).

\section{Abbildung 6 Größenstruktur der beteiligten Betriebe des Verarbeitenden Gewerbes im Vergleich mit der Grundgesamtheit}

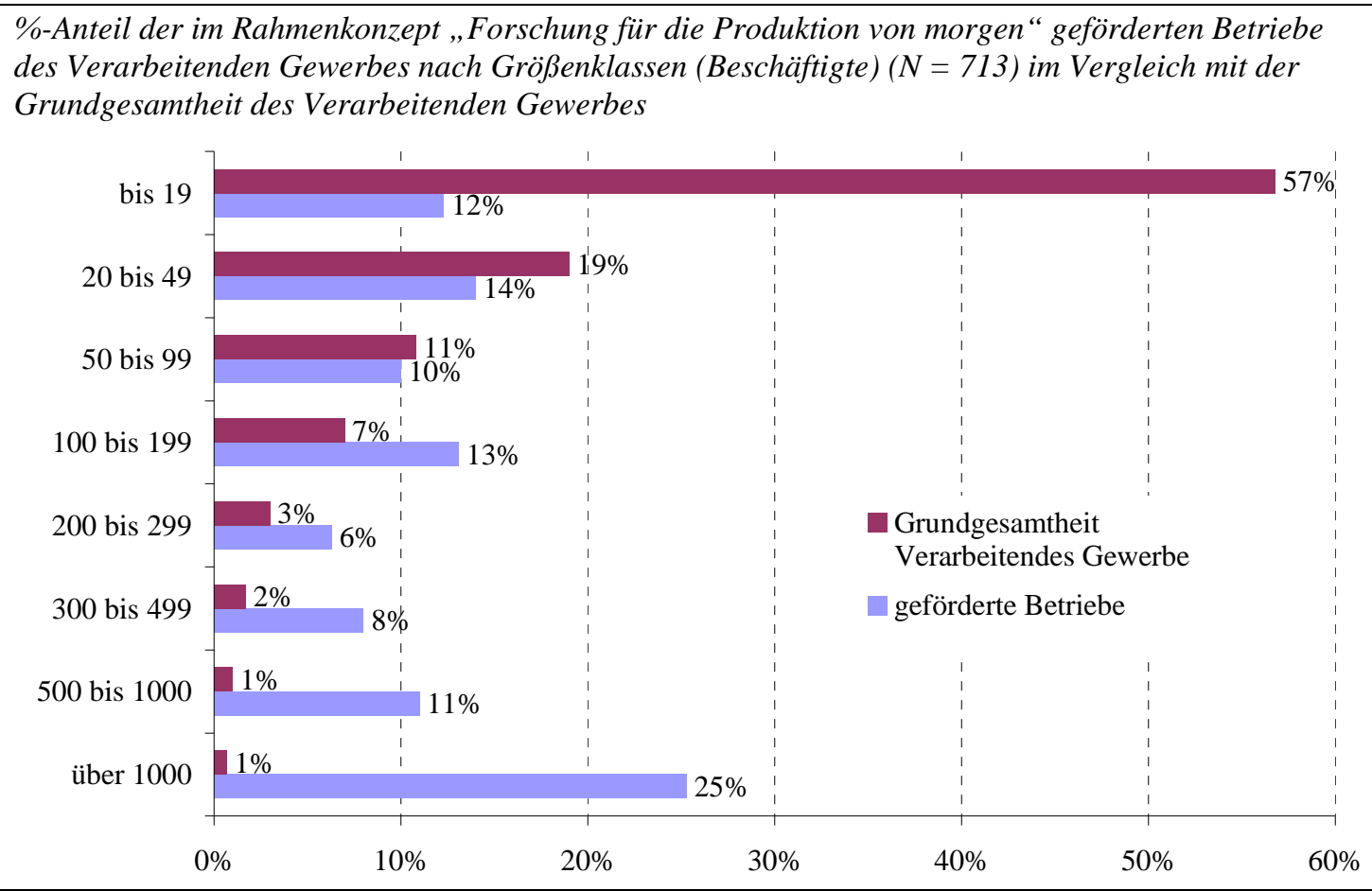

Quelle: Statistisches Bundesamt (Daten für das Jahr 2000); Berechnungen FhG-ISI, Darstellung Technopolis

Während auf Betriebe mit 500 und mehr MitarbeiterInnen nur etwa $2 \%$ der Grundgesamtheit entfallen, sind in den geförderten Verbundvorhaben ca. $36 \%$ Betriebe dieser Größenklasse vertreten. Dagegen stellen Betriebe mit weniger als 100 MitarbeiterInnen etwa $87 \%$ der Betriebe des Verarbeitenden Gewerbes, mit der Förderung wurden aber nur $36 \%$ dieser Betriebe erreicht. An dieser Stelle möchten wir jedoch festzuhalten, dass mit Mitteln der Forschungsförderung nicht alle relevanten Betriebe der potenziell infrage kommenden Zielgruppe zu gleichen Anteilen erreicht werden können. Die Verteilung der Förderung auf Betriebe unterschiedlicher Größen wird auch immer einen Kompromiss zwischen angemessener Berücksichtigung von Betrieben aller, insbesondere auch der kleineren Größenklassen sowie der für bestimmte Branchen bzw. Sektoren besonders wichtigen Betriebe, die im Regelfall größere oder Großunternehmen sind, suchen. Im Rahmenkonzept „Forschung für die Produktion von morgen" sieht die resultierende Verteilung schließlich so aus, dass Betriebe mit weniger als 100 MitarbeiterInnen (36\%), Betriebe mit 100 bis 500 MitarbeiterInnen (28\%) sowie Betriebe mit 500 und mehr MitarbeiterInnen (36\%) in etwa zu gleichen Anteilen beteiligt sind. Mit dieser Beteiligung der Industriebetriebe scheint es möglich, sowohl KMU im Vergleich zu anderen Förderprogrammen ausreichend zu beteiligen wie auch die wichtigen und größeren Unternehmen mit Vorreiterfunktion für ihre jeweiligen Sektoren bzw. Produktionsbedingungen in die Förderung angemessen mit einzubeziehen. 
Setzt man den KMU-Anteil der Fördermittel ins Verhältnis zum KMU-Anteil an den FuE-Gesamtaufwendungen im Verarbeitenden Gewerbe, dann zeigt sich folgende Relation: Von den insgesamt 166 Mio. EUR Fördermitteln für Industriebetriebe entfallen 131,5 Mio. EUR (79,3 \%) auf das Verarbeitende Gewerbes und davon etwa 75 Mio. EUR (57\%) auf Betriebe mit weniger als 250 Beschäftigten. Der mittlere jährliche Anteil von etwa 15 Mio. EUR über die Jahre 2000 bis 2004 beläuft sich im Verhältnis zu den 7 \% KMU-Anteil an den FuE-Gesamtaufwendungen des Verarbeitenden Gewerbes (2.753 Mio. EUR von 39.326 Mio. EUR) auf etwa 0,54 \%. Der Vergleichswert für größere Betriebe des Verarbeitenden Gewerbes mit 250 und mehr Beschäftigten beträgt bei einem Fördermittelanteil von $43 \%$ (56,5 Mio. EUR) und einem $93 \%$-Anteil an den FuE-Gesamtaufwendungen des Verarbeitenden Gewerbes (36.574 Mio. EUR) lediglich etwa 0,15 \%. Allerdings ist die Höhe der FuE-Aufwendungen kein ideales Maß zur Einschätzung der Größenklassenselektivität des BMBF Rahmenkonzepts, da nicht alle intendierten Problemlösungen (z. B. organisationsorientierte oder strategieorientierte Methoden und Konzepte) und einbezogenen Projektpartner (z. B. Anwender) notwendigerweise auf hinreichende FuE-Kapazitäten zurückgreifen müssen.

\section{Abbildung 7 Vergleich der Größenstruktur der geförderten Verarbeitenden Betriebe und Dienstleistungsbetriebe}

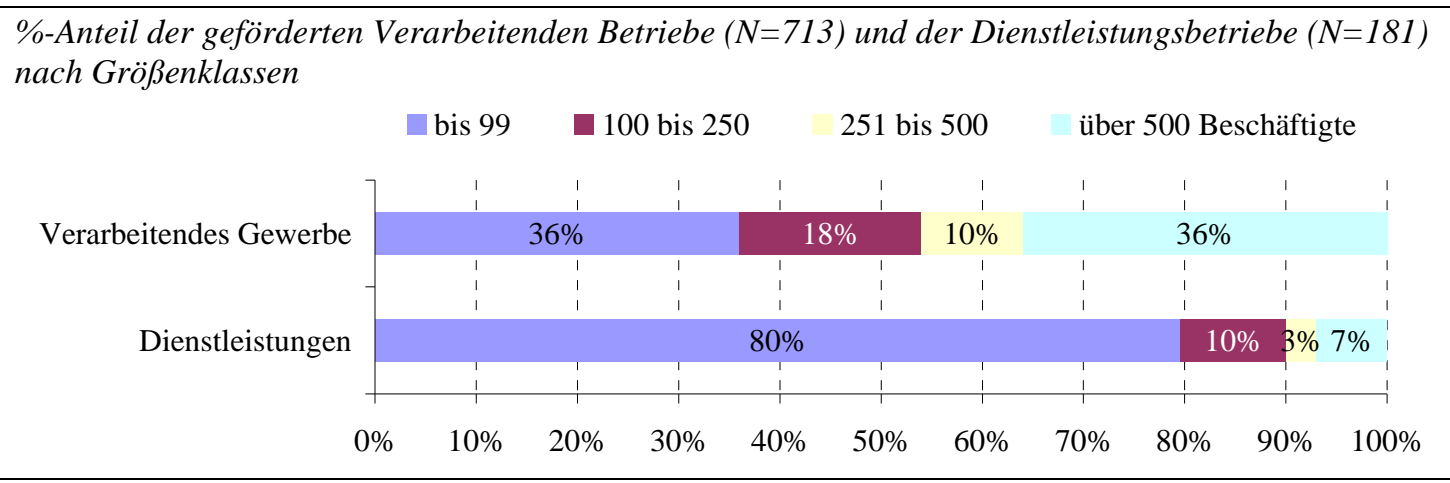

Quelle: PROFI Projektdatenauszug, Berechnungen FhG-ISI, Darstellung Technopolis

Wie vertiefende Analysen zeigen, unterscheidet sich die Größenstruktur der in den Verbundvorhaben beteiligten Industriebetriebe wesentlich danach, ob sie dem Verarbeitenden Gewerbe angehören oder den Sektoren wissensintensiver produktionsnaher Dienstleistung zuzurechnen sind (Abbildung 7). Von den insgesamt knapp $18 \%$ Unternehmen der Dienstleistungssektoren Datenverarbeitung und Datenbanken, Forschung und Entwicklung sowie Erbringung von Dienstleistung überwiegend für Unternehmen beschäftigen $90 \%$ weniger als 250 Beschäftigte und sind der Gruppe der KMU zuzurechnen. Auf der anderen Seite beträgt der KMUAnteil an den geförderten Betrieben des Verarbeitenden Gewerbes etwa $54 \%$. Diesen Befund gilt es bei der Interpretation der Quote der an den Verbundvorhaben des Rahmenkonzepts beteiligten KMU mit zu berücksichtigen. Zwar sind insgesamt $60 \%$ der geförderten Industriebetriebe als KMU zu identifizieren, in der vorrangigen Zielgruppe des Verarbeitenden Gewerbes ist ihr Anteil mit $54 \%$ jedoch etwas geringer. 


\section{Box 2: Welche Gründe sind für KMU besonders relevant sich an Verbund- forschung zu beteiligen?}

Wir haben in der Teilnehmerbefragung für ein breites Spektrum von möglichen Gründen die Bedeutung für die Beteiligung am Verbundprojekt abgefragt und die Antworten mittels Faktoranalyse zu vier Hauptzielen kondensiert: FuE-Risiko senken; Kompetenzen erweitern; Kooperationen stärken; technologisches Know-how nutzen.

Eine Analyse der Antworten der Teilnehmer nach Beschäftigtenzahl zeigt bei drei der vier Hauptziele statistisch signifikante Unterschiede zwischen kleinen und großen Unternehmen. Für KMU steht das Senken des wirtschaftlichen und technischen FuERisikos tendenziell eher im Zentrum als für Betriebe mit mehr als 250 Beschäftigten. Gleichzeitig verfolgen kleinere Betriebe mit der Teilnahme am Verbundvorhaben im höherem Ausmaß den Aufbau von Kooperationen mit anderen Partnern.

\section{Abbildung Box 2.1 Strategische Hauptziele der Industriepartner nach Betriebsgröße}

Standardisierte Abweichungen vom Mittelwert aller Industriepartner

(*) Mittelwertunterschiede zwischen Gruppen statistisch signifikant $(\mathrm{p}<0,01)$

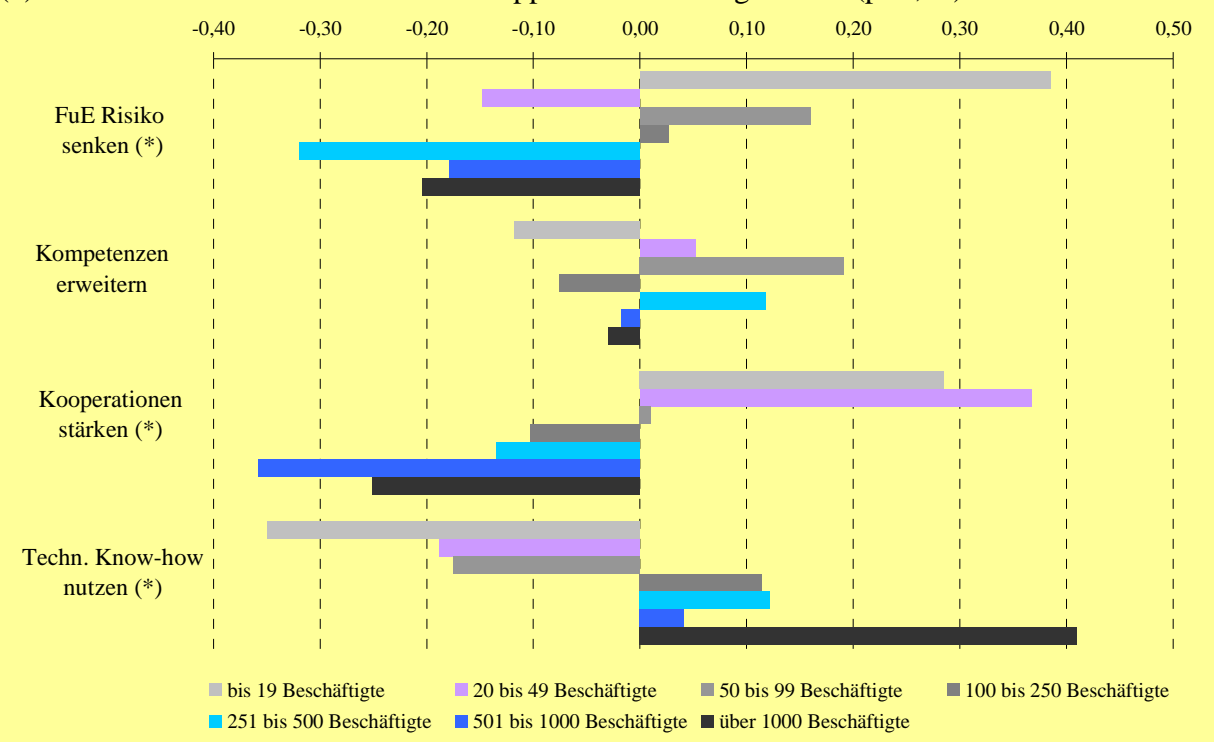

Quelle: TeilnehmerInnenbefragung ex-post Evaluation des BMBF Programms ,Forschung für die Produktion von morgen' für die Jahre 1999 bis 2004; Berechnungen und Darstellung Technopolis

Der Zugang und das Nutzen von externem technologischem Know-how (etwa durch Kontakte mit Universitäten und Forschungsinstituten und Zugang zu spezialisierter Technologie) ist andererseits für kleinere Unternehmen von geringerer Bedeutung. Ein strategischer Nutzen der Zusammenarbeit mit Forschungsinstituten scheint in erster Linie von Großunternehmen wahrgenommen zu werden, während bei kleineren Unternehmen unmittelbar projektrelevante Ziele sowie der Aufbau von Kooperationen mit anderen Industriebetrieben als Motive der Projektteilnahme dominieren.

Es gibt aber auch Anhaltspunkte dafür, dass ein wesentlicher Grund warum das Hauptziel, Technologisches Know-how nutzen' für KMU weniger von Bedeutung ist darin zu suchen ist, dass die teilnehmenden KMU selbst vielfach die technologischen Know-how Träger sind. Unter den KMU befinden sich überdurchschnittlich viele junge Unternehmen der WZ 72 bis 74 (DV und DB, FuE, Dienstleistungen für Unternehmen), die - so eine mögliche Hypothese - weniger Bedarf an zusätzlicher Kooperation mit Instituten sehen, da sie ohnehin aus deren Umfeld stammen. 
Ingesamt scheint es aber in den bis Ende 2004 im Rahmenkonzept „Forschung für die Produktion von morgen“" geförderten Verbundvorhaben gelungen zu sein, kleine und mittlere Unternehmen (KMU) breit zu beteiligen und damit Forschungsergebnisse für die Anwendung in KMU verfügbar zu machen.

\section{Beteiligung von Betrieben aus den neuen Bundesländern}

Im Rahmenkonzept wird neben der Anwendung der Forschungsergebnisse in kleinen und mittleren Unternehmen (KMU) auch das Ziel formuliert, mit den entwickelten Lösungen insbesondere Betriebe in den neuen Bundesländern zu unterstützen. Vor diesem Hintergrund haben wir analysiert, in welchem Umfang Industriebetriebe aus den neuen Ländern in die Verbundvorhaben integriert sind. Wie sich zeigt, sind von den insgesamt geförderten 894 Industriebetrieben 107 (das sind 12\%) in den neuen Bundesländern angesiedelt. Gemessen am Anteil an den Fördermitteln erhielten Betriebe aus den neuen Ländern 17,8 Mio. EUR, was 11 \% des Fördervolumens entspricht.

\section{Abbildung 8 Geförderte Verarbeitende Betriebe aus den alten und neuen Bundesländern}

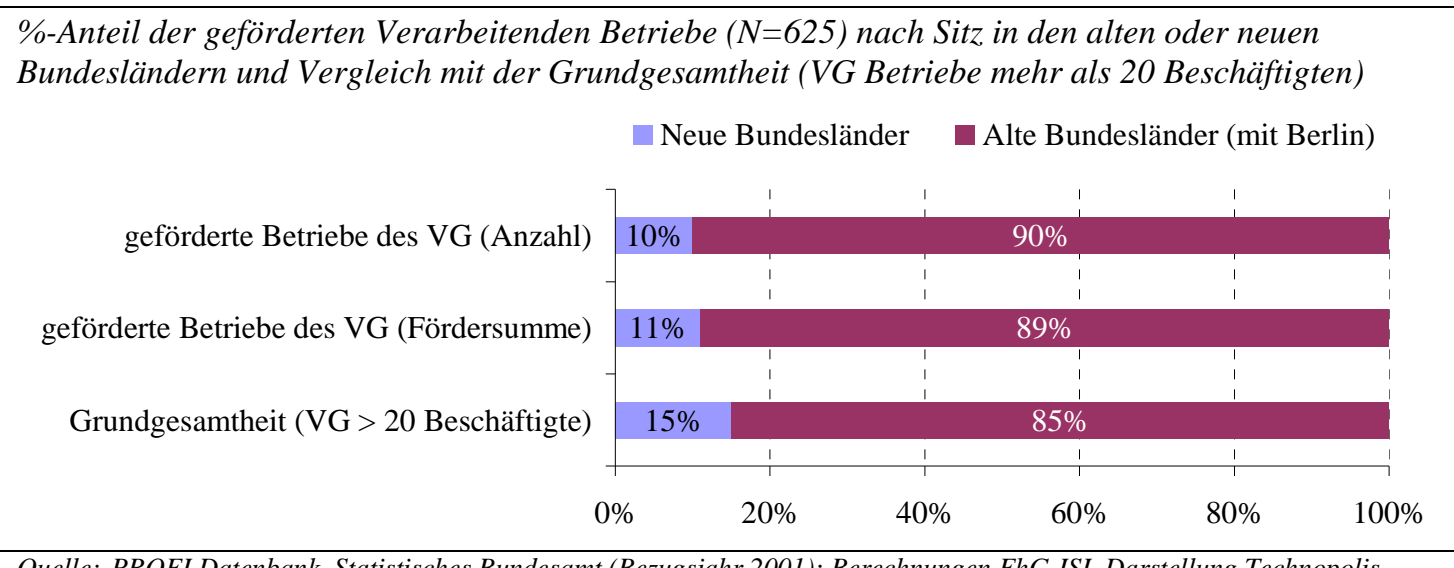

Quelle: PROFI Datenbank, Statistisches Bundesamt (Bezugsjahr 2001); Berechnungen FhG-ISI, Darstellung Technopolis

Vergleicht man diese Beteiligungsquoten ostdeutscher Betriebe für den statistisch vergleichbaren Ausschnitt des Verarbeitenden Gewerbes mit 20 und mehr MitarbeiterInnen mit der Gesamtanzahl der Betriebe dieser Klasse als einem verfügbaren $\mathrm{Maß}$ für die Grundgesamtheit, so stellt man fest, dass die entsprechende Quote bei den geförderten Betrieben etwa $10 \%$ beträgt und in der Grundgesamtheit bei etwa $15 \%$ liegt. Gemessen an der Anzahl werden Betriebe aus den neuen Bundesländern im Programm damit unterdurchschnittlich erreicht.

In diesem Kontext gilt es jedoch zu beachten, dass sich das Rahmenkonzept „Forschung für die Produktion von morgen“ insbesondere an Betriebe richtet, die eigene Forschung und Entwicklung betreiben. Als Hilfsgröße, um die Relation der betrieblichen Forschungsaktivitäten im Vergleich zwischen Betrieben der alten und neuen Bundesländer abzuschätzen, können wir den Anteil der internen betrieblichen FuE-Aufwendungen an den gesamten FuE-Aufwendungen des deutschen Verarbeitenden Gewerbes heranziehen. Der entsprechende Wert für das Jahr 2001 zeigt, dass Betriebe aus den neuen Bundesländern nur etwa 4,2 \% der internen FuE-Aufwendungen des Verarbeitenden Gewerbes erbringen. Im Vergleich zur anteiligen Forschungsleistung des Verarbeitenden Gewerbes in den neuen Bundesländern 
werde Betriebe aus den neuen Bundesländern am Rahmenkonzept „Forschung für die Produktion von morgen" überdurchschnittlich erreicht.

Vor dem Hintergrund des explizit geäußerten Ziels, Lösungen und Forschungsergebnisse besonders auch für Betriebe in den neuen Bundesländern bereitzustellen, stellt sich dennoch die Frage, ob es nicht wünschenswert sein könnte, mehr Industriebetriebe aus den neuen Bundesländern als bisher an den Verbundvorhaben zu beteiligen. Inwieweit dies aufgrund ihrer noch deutlich klein- und mittelständischer ausgeprägten Struktur oder im Hinblick auf benötigte Spezialkompetenzen möglich ist, können wir im Rahmen dieser Evaluation allerdings nicht beantworten.

\section{Beteiligung nach Projektrollen der Industriepartner}

Neben der Frage, welchen Branchen die in den Verbundvorhaben des Rahmenkonzepts „Forschung für die Produktion von morgen“ teilnehmenden Betriebe angehören und welche Größenklassenverteilung sie im Vergleich zur Grundgesamtheit des Verarbeitendes Gewerbes haben, ist auch interessant zu erfahren, in welchen Rollen die beteiligten Industriebetriebe und Forschungsinstitute in den Verbundvorhaben partizipieren. Diese Zuordnung zu typischen Rollenbildern in den Verbundprojekten kann nicht anhand der amtlichen Statistik geschehen, sondern basiert auf einer Analyse der Rahmenpläne aller 166 Verbundvorhaben. Im Vorfeld der Dokumentenanalyse wurden anhand der Erfahrungen der beteiligten Institute und der Kenntnis von etwa 35 Rahmenplänen von Verbundvorhaben des Rahmenkonzepts fünf prototypische Rollen für Industriebetriebe sowie zwei prototypische Rollen für Forschungsinstitute identifiziert. Dies sind für die Industriebetriebe:

- Der Entwickler, der in den zumeist technischen Entwicklungsprojekten eine zentrale Entwicklungsaufgabe wahrnimmt und um dessen zu entwickelnde Lösung herum sich das Verbundprojekt oder einzelne Teilprojekte des Verbundprojektes herum gruppieren.

- Der Entwickler für die eigene Anwendung, der in zumeist nicht technischen Verbundvorhaben sowohl bei der Entwicklung, Konzeption und Konfiguration der geplanten Lösung mitwirkt als auch diese Lösung in der Anwendung im eigenen Betrieb selbst testet, evaluiert und gegebenenfalls weiter anpasst.

- Der Anwender, dem in den Verbundvorhaben die Rolle zukommt, die zu entwickelnden technischen oder nichttechnischen Lösungen im eigenen Betrieb hinsichtlich ihrer technischen und wirtschaftlichen Machbarkeit zu evaluieren, zu Projektbeginn die wichtigsten Anforderungen und spezifischen Bedarfe zu formulieren und in die Lösungskonzeption einzubringen und damit als Korrektiv der Nachfrageseite die technische Entwicklung von Beginn bis zum Ende des Projektes in die richtige, weil praktisch anwendbare Richtung mitzulenken.

- Der Zulieferer, der für die zumeist technische Lösung des oder der Entwickler im Projekt wichtige Komponenten oder Baugruppen zur Erreichung des Gesamtziels des Vorhabens weiterentwickelt und im Projekt zur Verfügung stellt.

- Der Dienstleister, der Leistungen wie Softwareentwicklung, Datenbankentwicklung, Tests von Verfahren und Materialien, Konstruktions- und Entwicklungsaufgaben, Logistik- oder Beratungsexpertise etc. im Projekt zur Verfügung stellt und ggf. entsprechend den Anforderungen des Gesamtprojekts weiterentwickelt, um so zur zielorientierten Entwicklung der Gesamtlösung beizutragen. 
Die Institute können in den Verbundvorhaben folgende Rollen einnehmen:

- Forschungsinstitute, zumeist Universitäts-, Fachhochschul- oder FraunhoferInstitute, die zusammen mit den industriellen Partnern im Projekt die technische oder nicht technische Lösung hinsichtlich der Gesamtzielsetzung des Projektes weiterentwickeln und dafür ihre wissenschaftliche Expertise, Beratungsleistung und Möglichkeiten zur Durchführung von Prototypenentwicklung und Tests zur Verfügung stellen. Vielfach sind diese Forschungsinstitute auch für die Verallgemeinerung der entwickelten unternehmensspezifischen Lösung in (für eine breite Klientel nutzbare) Instrumente und Werkzeuge verantwortlich, insbesondere in nicht-technischen Entwicklungsprojekten.

- Transferpartner, die schwerpunktmäßig oder ausschließlich für die Aufbereitung und Verbreitung der wissenschaftlichen Erkenntnisse für eine spezifische Zielgruppe verantwortlich sind.

Die resultierende Rollenzuordnung der teilnehmenden Industriebetriebe und Institute eignet sich unserer Ansicht nach, um über alle 1.157 an den Verbundvorhaben beteiligten Partner ein belastbares Gespür dafür zu entwickeln, welche Rollen von welchen Industriebetrieben und Instituten schwerpunktmäßig eingenommen werden.

Von den industriellen Rollentypen entfällt auf den fokalen Entwickler mit knapp $20 \%$ der größte Anteil der Fördermittel (siehe Tabelle 6). Im Vergleich zu den anderen Rollentypen sind hier auch die jeweiligen Teilprojekte mit im Mittel etwa 283 Tsd. EUR Förderung am größten dimensioniert. Industriebetriebe mit der Rolle des fokalen Entwicklers sind in insgesamt 115 entsprechend $69 \%$ der Verbundvorhaben vertreten. Ein Großteil der geförderten Verbundvorhaben hat damit zumindest einen fokalen Entwickler auf Seiten der Industrie mit in das Konsortium integriert hat.

Industriebetriebe mit der Rolle des Entwicklers für die eigene Anwendung partizipieren zu etwa $13 \%$ an den Fördermitteln des Rahmenkonzepts. Diese Rolle ist mit im Mittel etwa 141 Tsd. EUR Förderung anteilmäßig mit den geringsten Projektvolumina ausgestattet. Zur Entwicklung insbesondere nicht-technischer Lösungen und Methoden mit stärkerem Fokus auf der Anwendungserprobung scheinen demnach kleinere Projektvolumina auszureichen. In diesem Kontext sollte man darauf achten, dass keine kritischen Massen unterschritten werden, die eine sinnvolle Projektbearbeitung über eine Laufzeit von zwei bis drei Jahren kaum mehr möglich machen. In 48 entsprechend $29 \%$ der Verbundvorhaben und damit in den vergleichsweise wenigsten Verbünden sind Firmen mit der Rolle des Entwicklers für die eigene Anwendung integriert. Dies erklärt sich insbesondere durch die Konstruktion der nicht-technischen Verbundvorhaben, in denen oftmals ein oder wenige Forschungsinstitute mit mehreren Industriebetrieben unterschiedlicher Branchenund Größenklassen zusammenarbeiten, um gemeinsam für eine breit gefächerte Klientel anwendbare Lösungen zu entwickeln. 
Tabelle 6 Förderung der Projektteilnehmer und Teilnahmen an Verbundvorhaben nach Projektrollen

\begin{tabular}{|c|c|c|c|}
\hline & $\begin{array}{c}\text { Anteil an den } \\
\text { Fördermitteln des } \\
\text { Rahmenkonzepts } \\
{[\%]}\end{array}$ & $\begin{array}{l}\text { Anzahl (und Anteil) } \\
\text { der Verbundprojekte, } \\
\text { in denen die Projekt- } \\
\text { rolle vertreten ist }\end{array}$ & $\begin{array}{l}\text { Anteil der Verbund- } \\
\text { projekte, in denen } \\
\text { eine der Projekt- } \\
\text { rollen vertreten ist }\end{array}$ \\
\hline Entwickler & 19,8 & $115(69 \%)$ & 145 \\
\hline Entwickler für die eigene Anwendung & 13,2 & $48(29 \%)$ & $(87 \%)$ \\
\hline Anwender & 9,3 & $77(46 \%)$ & $(72 \%)$ \\
\hline Dienstleister & 7,1 & $72(43 \%)$ & 116 \\
\hline Zulieferer & 13,4 & $68(41 \%)$ & $(70 \%)$ \\
\hline Industriebetriebe insgesamt & 62,8 & $163(98 \%)$ & \\
\hline Forschungsinstitute & 36,6 & $158(95 \%)$ & \\
\hline Transferpartner & 0,6 & $10(6 \%)$ & \\
\hline Institute insgesamt & 37,2 & $159(96 \%)$ & \\
\hline Insgesamt (in 166 Verbundprojekten) & 100 & & \\
\hline
\end{tabular}

Industrielle Anwender sind mit etwa $9 \%$ der Fördermittel in die Verbundvorhaben des Rahmenprogramms einbezogen. Die mittlere Projektgröße von Industriebetrieben dieser Rolle ist mit etwa 158 Tsd. EUR Förderung ebenfalls etwas unterdurchschnittlich. In 77 entsprechend $46 \%$ der Verbundvorhaben ist zumindest ein industrieller Anwendungspartner integriert. In etwa der Hälfte der Verbundvorhaben wird demnach durch das Einbinden konkreter Anwender darauf gezielt, die technische und wirtschaftliche Realisierbarkeit der zu entwickelnden Lösungen im Blick zu behalten.

Betrachtet man die industrielle Entwicklerseite der Verbundvorhaben integriert, so zeigt sich folgendes Bild: In 145 entsprechend $87 \%$ der Verbundvorhaben ist die Industrie als zentraler Akteur der Entwicklung eingebunden, d. h. zumindest ein Industriepartner ist fokaler Entwickler oder Entwickler für die eigene Anwendung. Dies ist ein Beleg für die insgesamt hohe Praxisorientierung, die entwicklungsseitig mit den Verbundvorhaben des Rahmenkonzepts angezielt wird. Auf der Nachfrageoder Anwenderseite findet sich in insgesamt 120 entsprechend $72 \%$ der Verbundvorhaben mindestens ein Industriebetrieb, der als industrieller Anwender oder Entwickler für die eigene Anwendung agiert. Aus den Fördermittelanteilen dieser beiden Rollen lässt sich ein „Gesamt-Anwenderanteil“ von 10 bis $15 \%$ an der Förderung schätzen ${ }^{3}$. Hier stellt sich die Frage, ob dieser Anteil bzw. ein $9 \%$ Förderanteil ,echter“ Pilotanwender, deren spezifische Probleme mit Hilfe der Neuentwicklungen gelöst werden sollen, ausreicht, um den technischen und wirtschaftlichen Marktbezug der entwickelten Lösungen sicherzustellen.

Auf Basis der Analyse der Rahmenpläne konnten weitere etwa 40 bis 45 assoziierte Anwender oder Anwender im Unterauftrag von anderen Institutionen ausgemacht werden, die sich an den Verbundvorhaben auf diesem Weg, gefördert oder nicht gefördert, beteiligt haben. Auf diese Weise wird in etwa 20 Verbundvorhaben versucht, den Anwenderbezug zu integrieren oder weiter zu stärken. Für die weitere Steuerung des Rahmenkonzepts sollte man im Auge behalten, ob der Einbezug assoziierter Anwender z. B. über ungeförderte Arbeitskreisen im Hinblick auf die

3 Unter der Annahme, dass der Anwenderanteil bei Industriebetrieben mit der Zwitterrolle des Entwicklers für die eigene Anwendung im Mittel zwischen $10 \%$ und $40 \%$ liegt. 
Marktorientierung der entwickelten Lösungen eine nachweislich wirksame Alternative zur direkten und geförderten Anwenderintegration sein kann.

Auf Industriebetriebe mit der Rolle des Zulieferers entfallen etwa $13 \%$ der Fördermittel des Rahmenkonzepts. Die mittlere Projektgröße dieses Rollentyps ist mit etwa 189 Tsd. EUR Förderung vergleichsweise groß. Firmen dieses Typus sind in 68 entsprechend $41 \%$ der Verbundvorhaben anzutreffen. Industriebetriebe mit der Rolle des Dienstleisters partizipieren zu weiteren $7 \%$ mit einer etwas unterdurchschnittlichen mittleren Projektgröße von 157 Tsd. EUR an Fördermitteln. Trotz dieser recht geringen finanziellen Beteiligung ist dieser Rollentypus in vergleichsweise vielen Verbundvorhaben (72 entsprechend $43 \%$ ) zu finden. Insgesamt zeigt sich, dass zusammen genommen 116 entsprechend $70 \%$ der Verbundvorhaben nicht ohne die Spezialkompetenzen gewisser Zulieferer von Komponenten und Baugruppen oder Dienstleister für Software-, Datenbanken-, IuK- oder FuE-Leistungen auszukommen scheinen.

Forschungsinstitute partizipieren zu etwa $37 \%$ an den Gesamtfördermitteln des Rahmenkonzept bei einer mittleren Projektgröße von 384 Tsd. EUR an Fördermitteln. Sie sind in 158 Projekten entsprechend $95 \%$ aller Verbundvorhaben vertreten und damit von allen Rollen in den vergleichsweise meisten Vorhaben integriert. Dies unterstreicht den Ansatz der Verbundvorhaben, in möglichst allen Projekten die fachlichen Kompetenzen von Wissenschaft und Industrie zusammen zu bringen und im Austausch fruchtbar werden zu lassen.

Etwa 0,6\% aller Fördermittel des Rahmenkonzepts entfallen auf die insgesamt elf geförderten Transferpartner, wobei die mittlere Projektgröße etwa 144 Tsd. EUR an Fördermitteln beträgt. Damit sind lediglich in zehn (entsprechend $6 \%$ der Verbundvorhaben) auf den Ergebnistransfer spezialisierte Institutionen integriert. Diese geringe Einbindungsquote muss vor dem Hintergrund des Ziels des Rahmenkonzepts und seiner Verbundvorhaben, die breite Anwendung der entwickelten Lösung insbesondere in KMU sicher zu stellen, kritisch hinterfragt werden. Wie die Analyse der Rahmenpläne zeigt, wirken in den Verbundvorhaben etwa 15 weitere Projektpartner als assoziierte Transferpartner oder Transferpartner im Unterauftrag mit. Damit erhöht sich die Zahl der Verbundprojekte mit Transferpartnern um etwa zehn weitere auf insgesamt etwa 20 entsprechend $12 \%$. Doch auch diese korrigierte Beteiligungsquote scheint vor dem Hintergrund der Zielsetzung der breiten industriellen Anwendbarkeit der entwickelten Lösungen weiterhin problematisch. Hier stellt sich die Frage, inwieweit ein wirksamer Transfer einerseits von Forschungsinstituten, die verallgemeinerbare Lösungen in die Öffentlichkeit tragen, sowie durch Industriebetriebe, die ihre entwickelten Produkte und Lösungen aktiv vermarkten, sichergestellt werden kann.

\section{Beteiligung der Forschungsinstitute}

Forschungsinstitute sind als Projektpartner, wie bereits zuvor dargestellt, in 158 der 166 und damit $95 \%$ der Verbundvorhaben integriert. Insgesamt partizipieren Forschungsinstitute $263 \mathrm{Mal}$ in den Verbundvorhaben. Bereinigt um Mehrfachteilnahmen lassen sich 144 verschiedene beteiligte Forschungsinstitute identifizieren (siehe Abbildung 9). 
Analysieren wir die beteiligten Forschungsinstitute nach Fachbereichen, so zeigt sich erwartungsgemäß, dass die überwiegende Mehrzahl dem Maschinenbau (105 entsprechend $40 \%$ ) zuzuordnen ist, gefolgt von der Verfahrenstechnik mit 52 (entsprechend $20 \%$ ). Diese Prozentzahlen entsprechen auch nahezu exakt dem Anteil der jeweiligen Fachbereiche an den gesamten Institutsfördermitteln des Rahmenkonzepts. Auf den Rangplätzen 3 und 4 der in den Verbundprojekten beteiligten Fachbereiche folgen die Elektrotechnik mit 33 Teilnahmen (13\%) sowie die Wirtschaftswissenschaften mit 27 Teilnahmen (10\%) Institute. Rangplatz 5 und 6 nehmen die Informatik mit 15 Teilnahmen $(6 \%)$ sowie die Sozialwissenschaften mit 10 Teilnahmen (4\%) ein. Die weiteren Fachbereiche sind mit Anteilen unter $2 \%$ der beteiligten Forschungsinstitute weitgehend vernachlässigbar, darunter auch die Fachbereiche Mathematik, Physik, Wirtschaftsingenieurwesen und Chemie.

\section{Abbildung 9 Institutsbeteiligung nach Fachbereichen}

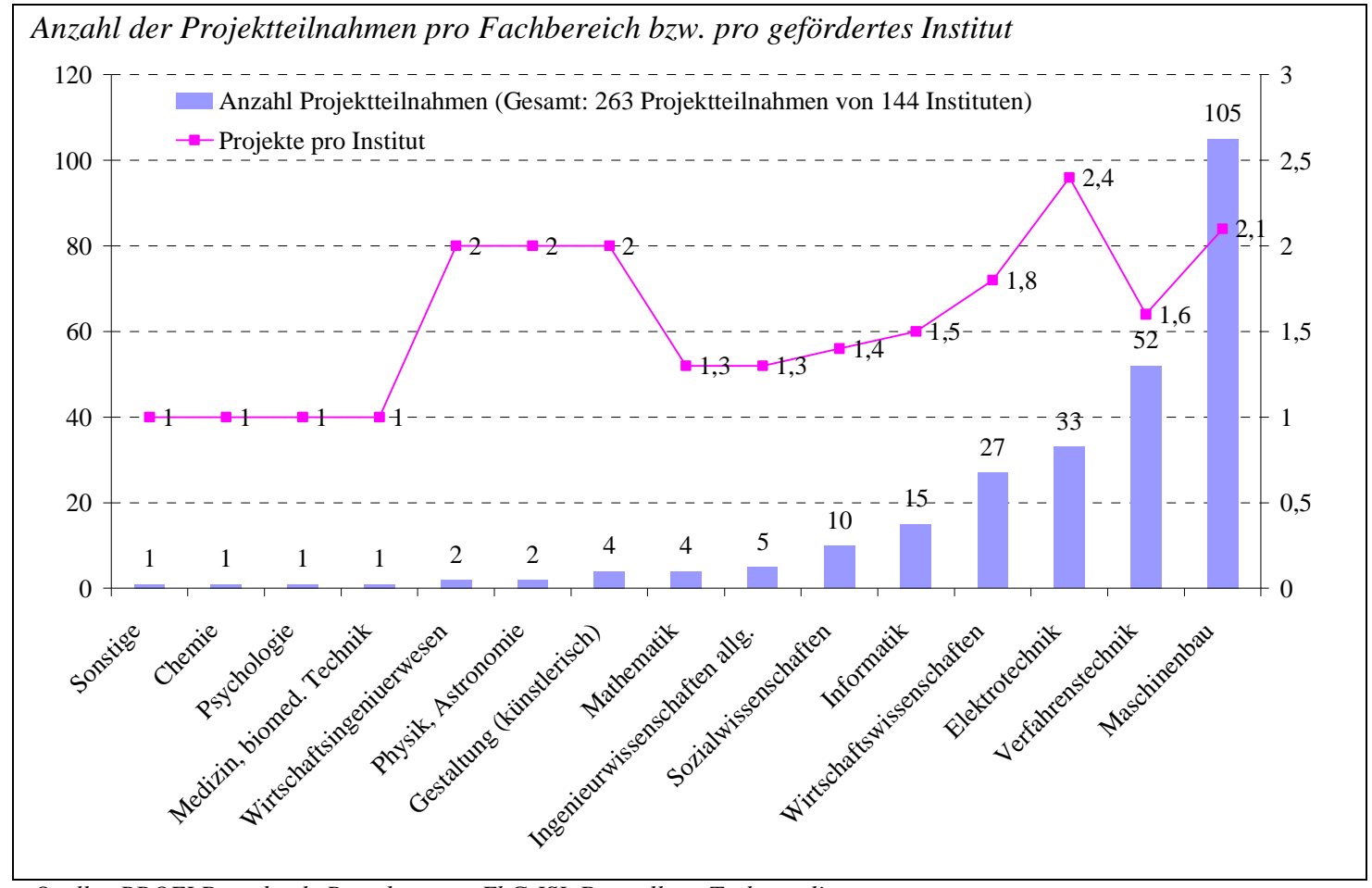

Quelle: PROFI Datenbank, Berechnungen FhG-ISI, Darstellung Technopolis

Eine vertiefende Analyse zeigt, dass insbesondere Forschungsinstitute der Fachbereiche Maschinenbau und Elektrotechnik mehrfach an den Verbundvorhaben des Rahmenkonzepts „Forschung für die Produktion von morgen“" teilgenommen haben. Im Durchschnitt hat im Fachbereich Maschinenbau jedes teilnehmende Institut an 2,1 Verbundprojekten und im Fachbereich Elektrotechnik an 2,4 Verbundvorhaben mitgearbeitet. Diese Quote liegt merklich über der Mehrfachteilnahmequote anderer Fachbereiche, beispielsweise von Instituten der Verfahrenstechnik mit im Mittel 1,6 Teilnahmen.

Vor dem Hintergrund der analysierten Institutsbeteiligung stellt sich die Frage, wie diese im Verhältnis zur Grundgesamtheit, gemessen am Fachbereichsanteil an den gesamten Drittmitteleinnahmen über alle Fachbereiche, einzuordnen ist. Erwartungsgemäß zeigt sich, dass die Institute der Fachbereiche Maschinenbau und Verfahrenstechnik nicht nur mit 60 \% der Institutsfördermittel am intensivsten am Rahmenkonzept partizipieren, sondern sind mit einem Anteil von $28 \%$ aller Hochschul- 
drittmittel auch jenseits des Rahmenkonzepts deutlich am stärksten drittmittelorientiert agieren (siehe Tabelle 7. Die mittleren jährlichen Fördermittel des Rahmenkonzepts (2000-2004) tragen bei den Instituten dieser Fachbereiche etwas mehr als $3 \%$ zu deren gesamten Drittmitteleinnahmen bei. Auch die Forschungsinstitute der Fachbereiche Elektrotechnik (15,5 \% Fördermittelanteil) und Wirtschaftswissenschaften (10\% Fördermittelanteil) sind in den Verbundvorhaben vergleichsweise intensiv eingebunden, da sie mit $11 \%$ bzw. $7 \%$ aller Hochschuldrittmittel insgesamt nicht so stark auf Drittmittel orientiert sind wie der Maschinenbau und die Verfahrenstechnik. Dennoch liegen sie auch bei der Drittmittelorientierung insgesamt im Vorderfeld über alle Fachbereiche. Die mittleren jährlichen Fördermittel des Rahmenkonzepts tragen jeweils gut $2 \%$ zur Drittmittelfinanzierung der Fachbereiche Elektrotechnik und Wirtschaftswissenschaften bei. Neben dem Fachbereich allgemeine Ingenieurwissenschaften sind auch die Sozialwissenschaften zu jeweils etwa $3 \%$ der gesamten Institutsfördermittel in die Verbundvorhaben des Rahmenkonzepts integriert. Auch für diese Fachbereiche trägt die Förderung im Rahmenkonzept jeweils etwa 2 bis $3 \%$ zu ihren Drittmitteleinnahmen bei.

\section{Tabelle $7 \quad$ Vergleich der Institutsfördermittel nach Fachbereichen mit den Anteilen an den Drittmitteleinnahmen aller Fachbereiche}

\begin{tabular}{lcccc}
\hline Fachbereich (FB) & $\begin{array}{c}\text { Drittmittel- } \\
\text { einnahmen }\end{array}$ & $\begin{array}{c}\text { Anteil an den } \\
\text { Drittmittelein } \\
\text { nahmen aller } \\
\text { FB [\%] }\end{array}$ & $\begin{array}{c}\text { Fördermittel } \\
\text { anteil im } \\
\text { Programm } \\
{[\%]}\end{array}$ & $\begin{array}{c}\text { Programm- } \\
\text { anteil an den } \\
\text { Drittmitteln* } \\
{[\%]}\end{array}$ \\
\hline Maschinenbau / Verfahrenstechnik & 362,2 & 27,7 & 59,6 & 3,2 \\
Physik, Astronomie & 207,8 & 15,9 & 0,7 & 0,1 \\
Elektrotechnik & 144,7 & 11,0 & 15,5 & 2,1 \\
Chemie & 141,4 & 10,8 & 0,2 & $<0,1$ \\
Humanmedizin & 118,2 & 9,0 & 0,3 & 0,1 \\
Informatik & 98,1 & 7,5 & 5,2 & 1,0 \\
Wirtschaftswissenschaften & 88,6 & 6,8 & 10,0 & 2,2 \\
Mathematik & 42,6 & 3,3 & 1,1 & 0,5 \\
Psychologie & 39,2 & 3,0 & 0,2 & 0,1 \\
Ingenieurwissenschaften allg. & 26,5 & 2,0 & 2,6 & 1,9 \\
Sozialwissenschaften & 20,3 & 1,5 & 2,9 & 2,9 \\
Wirtschaftsingenieurwesen & 15,9 & 1,2 & 0,5 & 0,6 \\
Gestaltung (künstlerisch) & 4,3 & 0,3 & 1,1 & 4,9 \\
\hline Gesamt & $1.309,6$ & 100 & 100 & 1,5 \\
\hline Quelle: Statistisches Bundesamt, Fachserie 11, Reihe 4.5, 2002. Berechnung FhG-ISI: * Berechnet als Anteil der Jahresmittel-
\end{tabular}

Dagegen sind Forschungsinstitute der Fachbereiche Physik/Astronomie und Chemie zu vergleichsweise sehr geringen Förderanteilen an den Verbundvorhaben des Rahmenkonzepts beteiligt (0,7 \% bzw. 0,2 \%). Dies liegt für den Fachbereich Physik und Astronomie auch inhaltlich nahe, da dieser sicherlich keine vorrangige Zielgruppe ist, auch wenn die angewandte Physik in manchen Bereichen Anknüpfungspunkte zur Produktionsforschung aufweist. Hinsichtlich der vergleichsweise geringen Beteiligung der Forschungsinstitute aus dem Fachbereich Chemie könnte man aber durchaus fragen, ob deren Kompetenzen nicht stärker in die Verbundforschung für innovative Produktions- und Verfahrenslösungen einbezogen werden könnten. Hier bestätigt sich das bereits bei der Analyse der industriellen Verbund- 
projektteilnehmer gewonnene Bild, dass Forschung für konkrete Problemlösungen der Chemischen und Prozessindustrie im Rahmenkonzept bislang eine eher untergeordnete Rolle spielt. Auf der anderen Seite hat man auf diese Weise mit den begrenzten Fördermitteln die Möglichkeit, bewusst Schwerpunkte in den in Deutschland traditionell starken Forschungs- und Wirtschaftsbereichen des Maschinenbaus und der Verfahrenstechnik zu setzen.

Insgesamt trägt die Förderung des Rahmenkonzepts „Forschung für die Produktion von morgen" etwa 1,5\% zu den gesamten Drittmitteleinnahmen der Hochschulen über alle Fachbereiche bei. Lassen wir den Ausreißer Gestaltung außer Betracht, so finden wir das Maximum von etwas mehr als $3 \%$ der Drittmittel wie zu erwarten in den Fachbereichen Maschinenbau und Verfahrenstechnik. Im Vergleich zur noch deutlich stärker begrenzten Stimulierung der Industriebetriebe des Verarbeitenden Gewerbes, bei denen die Förderung nur etwa 0,08 \% der jährlichen FuE-Aufwendungen ausmacht, ist die Institutsstimulierung zwar merklich höher. Dennoch gilt auch für die Forschungsinstitute der Befund, dass mit den begrenzten Fördermitteln selbst die Fachbereiche, auf die das Rahmenkonzept vorrangig zielt, nicht grundlegend gefördert werden können.

Begründet durch den Forschungsgegenstand des Rahmenkonzepts „Forschung für die Produktion von morgen" kommen als teilnehmende Forschungsinstitute insbesondere solche in Frage, die zur wissenschaftlichen Gesellschaft für Produktionstechnik (WGP) oder zum Produktionsverbund der Fraunhofer-Gesellschaft gehören. Wie sich zeigt, haben 21 der 34 WGP-Institute (62\%) sowie sechs der acht Institute des Fraunhofer-Verbunds Produktion (75\%) zumindest an einem Verbundvorhaben des Rahmenkonzepts teilgenommen (Abbildung 16). Zusammengenommen vereinen diese Forschungsinstitute etwa $33 \%$ der insgesamt 80 Mio. EUR an Fördermitteln des Rahmenkonzepts, die bis Ende 2004 für Forschungsinstitute bewilligt wurden.

\section{Schwerpunkte der technologischen Kompetenzen der teilnehmenden Institute}

Welche innovativen technologischen Kompetenzen in den Verbundvorhaben des Rahmenkonzepts von besonderer Bedeutung sind, zeigt auch eine Schlagwortanalyse der Kompetenzbereiche der beteiligten Forschungsinstitute. So sind Forschungsinstitute, deren Forschungsprofil den Werkstofftechnologien zuzuordnen ist, in 22 Verbundvorhaben beteiligt (entsprechend $14 \%$ der insgesamt 158 Verbundvorhaben mit Forschungsinstitutsbeteiligung). Institute mit ausgewiesenen Kompetenzen in den Bereichen Lasertechnik oder Mikrotechnik bzw. -elektronik sind in jeweils 16 Verbundvorhaben (entsprechend $10 \%$ der Projekte mit Forschungsinstitutsbeteiligung) integriert. In weiteren sieben Verbundvorhaben (entsprechend $4 \%$ der Verbundprojekte mit Institutsbeteiligung) finden sich Forschungsinstitute mit Kompetenzen in den Bereichen Oberflächen- oder Beschichtungstechnik. Explizit ausgewiesene Mechatronikkompetenz findet sich in weiteren drei Verbundvorhaben. Damit entfällt etwa ein Viertel der 263 Institutsbeteiligungen im Rahmenkonzept auf diese innovativen technologischen Kompetenzen. Das im Rahmenkonzept formulierte Ziel, bei der Entwicklung neuer fertigungstechnischer Lösungen gezielt Kompetenzen aus den Bereichen neue Werkstoffe und Oberflächen zu integrieren, scheint also in den geförderten Projekten aufgegriffen zu werden. 


\section{Interdisziplinäre Zusammenarbeit zwischen Forschungsinstituten}

Vor dem Hintergrund der Zielsetzung des Rahmenkonzepts ist es zudem interessant zu analysieren, ob und in welchem Umfang Forschungsinstitute verschiedener Wissenschaftsdisziplinen in Verbundvorhaben zusammenarbeiten. Demnach kooperieren in 57 der 158 Verbundprojekte mit Beteiligung von Forschungsinstituten (entsprechend $35 \%$ ) Institute aus verschiedenen Fachbereichen. Erwartungsgemäß am häufigsten arbeiten Institute der Fachbereiche Maschinenbau und Verfahrenstechnik zusammen, die in 13 Verbundvorhaben gemeinsam vertreten sind. Rangplatz 2 der interdisziplinären Forschungskooperationen belegt mit sechs Verbundvorhaben die Fachbereichskombination Maschinenbau und Elektrotechnik, gefolgt von Maschinenbau und Wirtschaftswissenschaften (Rang 3) mit fünf gemeinsamen Verbundvorhaben und Verfahrenstechnik und Elektrotechnik (Rang 4) mit vier gemeinsamen Verbundvorhaben. Auf den weiteren Plätzen folgt die interdisziplinäre Verbundforschung der Fachbereiche Maschinenbau und Sozialwissenschaften, Wirtschaftswissenschaften und Sozialwissenschaften sowie Maschinenbau und allgemeines (sonstiges) Ingenieurwesen mit jeweils drei Nennungen.

Insgesamt gelingt es damit in etwa einem Drittel der Verbundprojekte mit Forschungsinstitutsbeteiligung, Kompetenzen verschiedener Fachbereiche interdisziplinär zusammenzuführen. Auf der anderen Seite stützen sich zwei Drittel der Verbundvorhaben mit Institutsbeteiligung auf Kompetenzen aus einem einzelnen Fachbereich, insbesondere Maschinenbau und Verfahrenstechnik. 


\section{Programmwirkungen}

In diesem Abschnitt geht es primär um die wirtschaftlichen und technologischen Effekte der Programmteilnahme. Einerseits stellen wir uns die Frage nach den direkten Kommerzialisierungserfolgen des Programms, beispielsweise im Hinblick auf neue bzw. verbesserte Produkte, Prozesse und Dienstleistungen. Generell stellen wir die Frage nach der Verwertung der Projektergebnisse bzw. zu bestehenden Hemmnisse der Verwertung. Wir fragen in diesem Abschnitt auch nach dem Einfluss der Projektteilnahme auf das Innovationsverhalten und die technologische Kompetenz der Unternehmen und in wie fern mit der Teilnahme an der Fördermaßnahme die Unternehmen bestehende Innovationshemmnisse bewältigen konnten. Schließlich interessieren uns Fragen nach den Auswirkungen der Verbundprojekte auf sozioökonomische Dimensionen wie Wettbewerbsfähigkeit und Arbeitsplätze.

Als empirische Grundlage für unsere Auswertungen in diesem Abschnitt ziehen wir verschiedene Quellen heran. Zum einen stehen uns die Antworten der Teilnehmer zu den Wirkungen der Projektteilnahme aus der on-line Befragung zur Verfügung. Zusätzlich können wir die Ergebnisse der vom FhG-ISI getrennt durchgeführten Erhebung Innovationen in der Produktion für die Jahre 2003 und 2006 für einen Vergleich zwischen geförderten und nicht geförderten Betrieben hinsichtlich zentraler Input- und Output-Innovationsindikatoren heranziehen. Dies ermöglicht es uns vergleichsweise robuste Aussagen über die Wirksamkeit des Programms abzugeben. Schließlich stehen uns als zusätzliche qualitative Quellen die Ergebnisse aus den Interviews mit beteiligten Unternehmen und ExpertInnen, insbesondere aus den drei durchgeführten Fallstudien sowie die Auswertung von insgesamt 160 Schluss- und Erfolgskontrollberichten abgeschlossener Vorhaben bei KMU zur Verfügung.

\subsection{Additionalität und Anstoßwirkung der Projektförderung}

Mit der vorwettbewerblichen Förderung von FuE-Vorhaben soll eine möglichst hohe Wirkung der eingesetzten öffentlichen Mittel erreicht werden. Forschungsförderung soll die Akteure dazu anregen, zusätzliche FuE Anstrengungen zu unternehmen. Das Zusätzliche lässt sich in der Programmförderung nicht unbedingt rein finanziell bewerten, sondern bezieht sich auch auf Additionalitäten bei der Orientierung und den Ambitionen der Forschungsvorhaben, auf Kooperationsadditionalität (d.h. es wird mit Partnern kooperiert mit denen sonst nicht zusammengearbeitet worden wäre) bzw. die Forschungsvorhaben könnten später bzw. in geringerem Umfang in Angriff genommen werden. Wenn keines dieser Kriterien erfüllt wird, ist die Förderung für den Geförderten eine reine finanzielle Mitnahmeleistung.

(Teil-)Mitnahmeeffekte können in der Forschungsförderung nie völlig ausgeschlossen werden. Ein geringes Ausmaß an Mitnahmeverhalten auf Seiten der Förderakteure muss förderpolitisch auch akzeptiert werden, da es wünschenswert und notwendig ist, dass die FuE-Vorhaben in den beteiligten Industriebetrieben eine hohe Priorität zukommt, die Intention zur Durchführung der FuE Arbeiten also auch ohne Förderung gegeben ist. Angestrebte strukturelle Effekte der Verbundförderung (etwa Sicherstellung der Marktrelevanz der FuE-Arbeiten durch Einbeziehung von Anwendern) können es forschungs- und technologiepolitisch opportun erscheinen lassen, auch Partner zu fördern, die zumindest mit einer gewissen Wahrscheinlichkeit auch ohne Förderung thematisch vergleichbare Arbeiten durchgeführt hätten. 
Gleichzeitig ist klar, dass Mitnahmeeffekte möglichst gering und die Additionalität des Programms möglichst hoch sein sollten.

Die Bestimmung des Ausmaßes und die Interpretation von Additionalitäten ist schwierig. Zur Abschätzung der Additionalität haben wir in der Teilnehmerbefragung die kontrafaktische Frage gestellt, welches Schicksal die Projektskizzen genommen hätte, wäre es zu keiner Förderung im Rahmen des BMBF Programms ,Forschung für die Produktion von morgen' gekommen. Nach den Angaben der Industriepartner wären 56\% der FuE-Vorhaben ohne die Förderung nicht durchgeführt worden. Insgesamt $16 \%$ gaben an, sie hätten das Vorhaben mit anderen Fördermitteln oder ohne Änderung ( d.h. im Rahmenkonzept zu einem späteren Zeitpunkt) versucht zu finanzieren. In Summe 3\% der Antwortenden machten keine Angabe zur Finanzierung. Ein Viertel (25\%) der Industriepartner gab an, sie hätten das Vorhaben im Falle der Nicht-Förderung aus Eigenmitteln finanziert.

\section{Abbildung 10 Additionalität der Projektförderung}

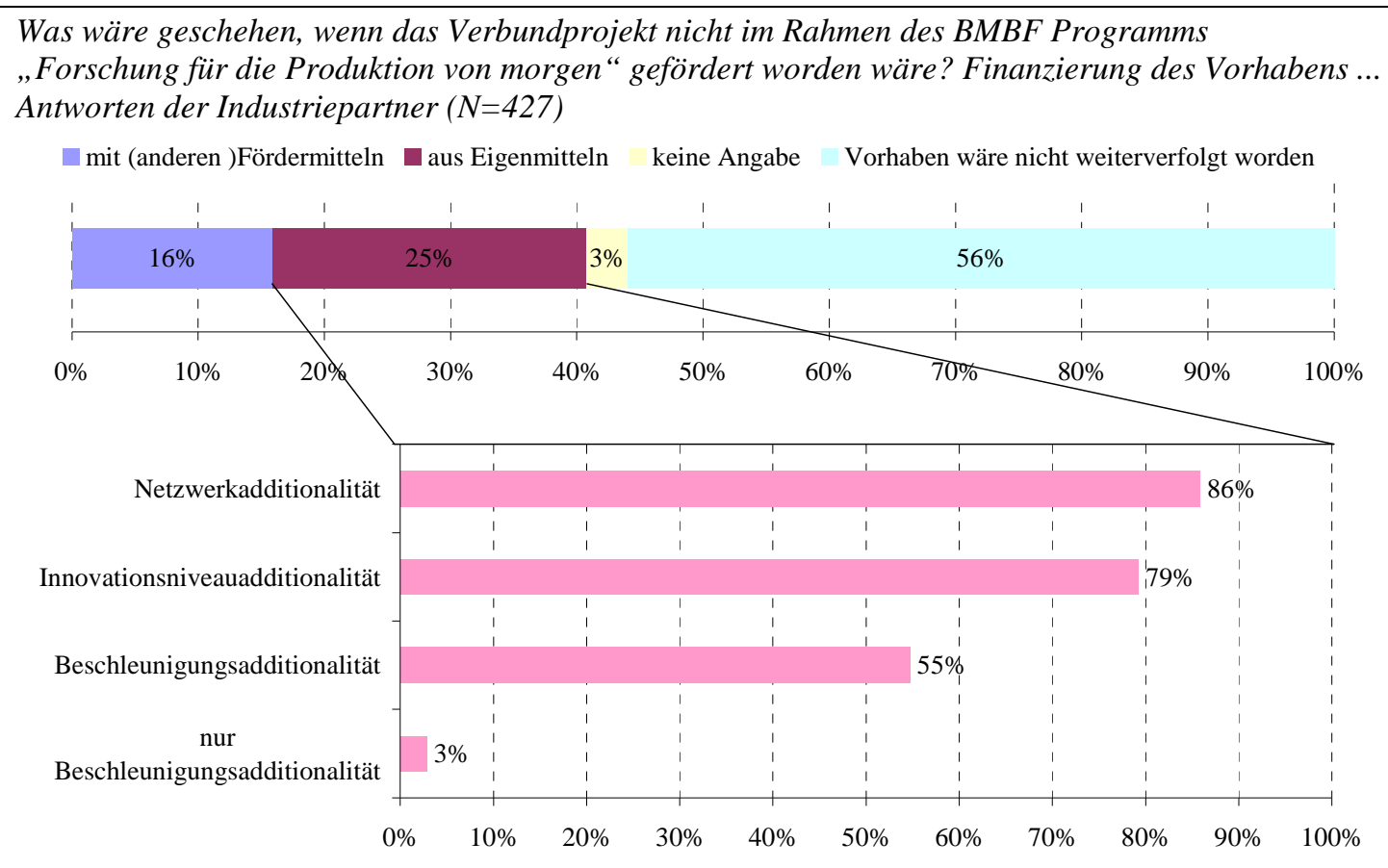

Quelle: TeilnehmerInnenbefragung ex-post Evaluation des BMBF Programms, Forschung für die Produktion von morgen' für die Jahre 1999 bis 2004; Berechnungen und Darstellung Technopolis. Anmerkungen: Netzwerkadditionalität: Durchführung im Falle der Nicht-Förderung ohne Forschungsinstitute, Entwicklungspartner und Anwender; Innovationsniveauadditionalität: Durchführung im Falle der Nicht-Förderung in geringerem Umfang und mit weniger ambitionierten Zielen; Beschleunigungsadditionalität: spätere Durchführung im Falle der Nicht-Förderung.

Wir haben für letztere Gruppe von Unternehmen im Detail geprüft, ob und welche Veränderungen durch die Förderung ausgelöst wurden. Dabei zeigt sich, dass sich in jedem einzelnen der 106 Fälle in dieser Gruppe ein Additionalitätseffekt feststellen lässt. In 91 Fällen (86\%) kann ein Netzwerk-Effekt der Förderung festgestellt werden (d.h. Durchführung im Falle der Nicht-Förderung ohne Forschungsinstitute, Entwicklungspartner und Anwender), in 58 Fällen (55\%) eine Beschleunigungswirkung (d.h. spätere Durchführung im Falle der Nicht-Förderung) und in 84 Fällen (79\%) ein positiver Einfluss auf das Innovationsniveau (d.h. Durchführung im Falle der Nicht-Förderung in geringerem Umfang und mit weniger ambitionierten Zielen). Nur in drei der 106 Fälle (3\%) lassen die Antworten ausschließlich auf einen Beschleunigungseffekt schließen, nicht jedoch auf einen Netzwerk- bzw. Innovations- 
niveaueffekt. Eine Additionalität der Förderung ist damit jedenfalls auch bei all jenen Industriepartnern gegeben, die ihr FuE-Vorhaben vollständig eigenfinanziert durchgeführt hätten.

Als einen weiteren Anhaltspunkt, in welchem Ausmaß die Förderung der Vorhaben im Rahmenkonzept „Forschung für die Produktion von morgen“ eine Anstoßwirkung gezeigt hat bzw. Kooperationen zwischen Innovationsakteuren stabilisieren halfen, können wir auch die Antworten der Befragten zur Weiterführung der Entwicklungsarbeiten nach Ende der FuE-Vorhaben heranziehen.

\section{In welchem Ausmaß werden die Entwicklungen nach Projektende weitergeführt und auf andere Themen ausgedehnt?}

Bei bereits abgeschlossenen FuE-Vorhaben haben wir in der Befragung danach gefragt, ob und in welcher Form die Entwicklungen nach Ende des Verbundprojekts weitergeführt wurden. Dabei zeigt sich, dass vier Fünftel der Industriepartner und mehr als 90\% der Institutspartner angaben, die Entwicklungen nach Ende des Verbundprojekts weitergeführt zu haben (Abbildung 11). Während die Institutspartner überwiegend wieder mit Partnern aus den Verbundprojekten kooperieren (44\%) ist bei Industriepartnern die Weiterentwicklung ohne Partner die häufigste Form der Fortsetzung der FuE-Arbeiten (41\%). In etwa einem Drittel der Fälle (31\%) werden die FuE-Arbeiten aber mit Partnern aus dem Verbund weitergeführt.

\section{Abbildung 11 Weiterführung der Entwicklungen nach Projektabschluss}

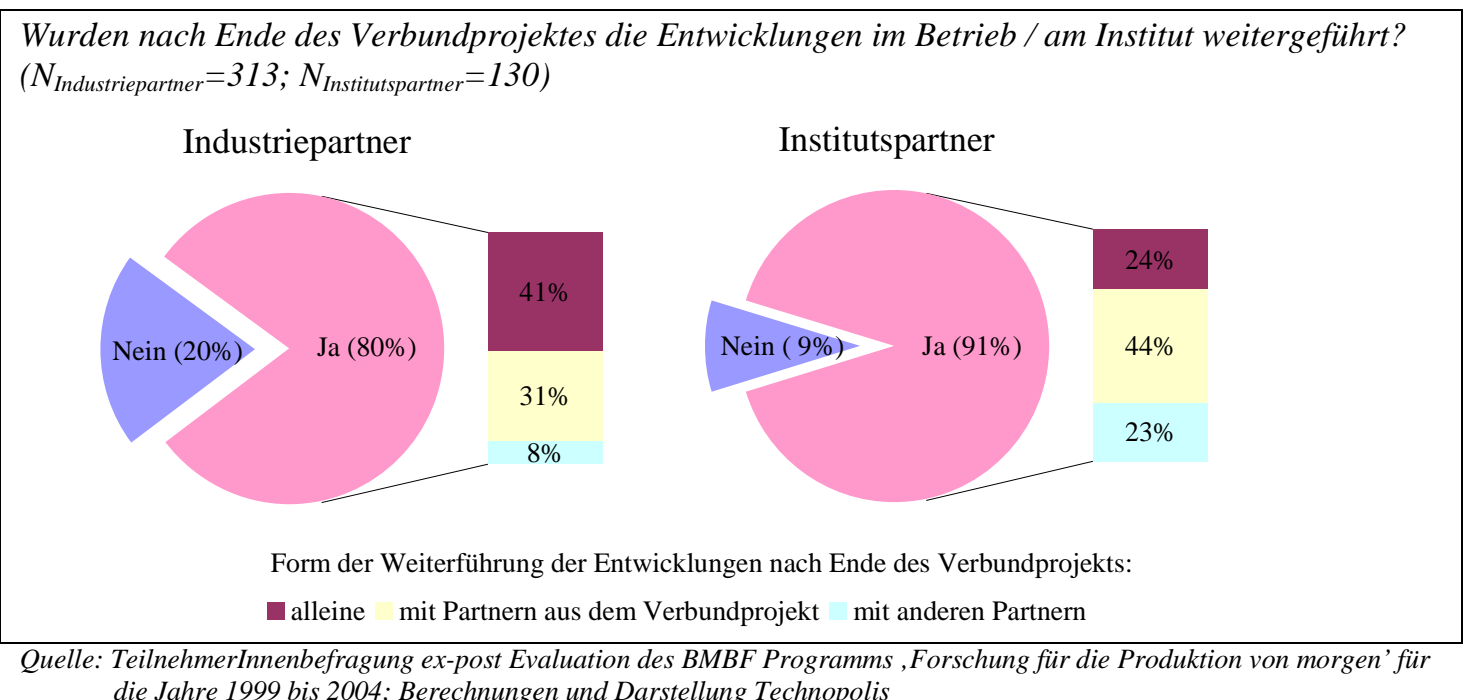

Die Weiterentwicklung mit anderen Partnern spielt bei der Industrie mit 8\% der Nennungen eine geringe Rolle, während die Institute in 23\% der Fälle angaben, die Entwicklungen mit anderen Partnern fortgesetzt zu haben. Dieses Ergebnis ist ein Anhaltspunkt dafür, dass Institute eine Transferfunktion der Projektergebnisse durch die Einbindung neuer Partner in die Weiterentwicklung wahrnehmen.

Die Ergebnisse der Teilnehmerbefragung zeigen auch, dass die Verbundprojekte die Zusammenarbeit zwischen den Partnern über das ursprüngliche Thema des Projekts hinaus festigen und Kooperationen auf andere Bereiche ausgeweitet werden (siehe Abbildung 12). 52\% der Industriepartner gaben an, heute mit ehemaligen Institutspartnern in anderen FuE-Vorhaben zusammen zu arbeiten. Immerhin $42 \%$ der 
Industriepartner arbeiten mit ehemaligen Partnern aus der Wirtschaft in anderen FuE-Vorhaben zusammen. Die häufigste Kooperationsform nach Ende des Verbundprojekts untereinander ist allerdings informeller Natur: $61 \%$ der Betriebe und $71 \%$ der Institute unterhalten heute mit ehemaligen Industriepartnern informelle Kontakte, beispielsweise Informationsaustausch oder Benchmarking. Mehr als drei Viertel der KMU führen ihre Kooperationen mit Industriepartnern nach Ende des Verbundprojekts fort, fast die Hälfte davon in Form von neuen FuE-Kooperationen.

\section{Abbildung 12 Weiterführung der Kooperationen in anderen Bereichen}

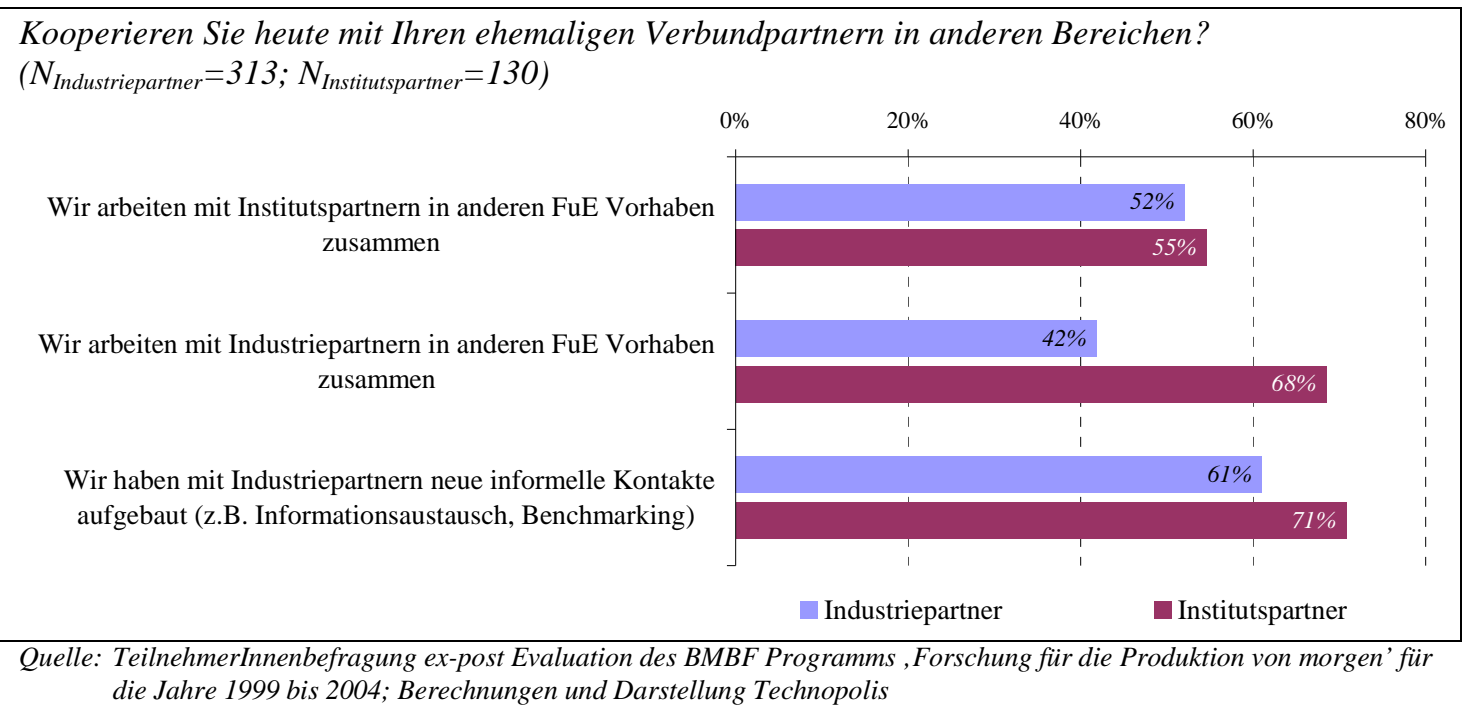

Insgesamt deuten diese Ergebnisse auf nachhaltige, stabile Kooperationsbeziehungen zwischen den Institutspartnern und Industriepartnern, aber auch zwischen den Industriepartnern untereinander hin, die durch die Verbundprojekte gestärkt werden.

\section{Werden durch die Förderung im Rahmenkonzept zusätzliche FuE-Aufwendungen in den Betrieben ausgelöst?}

Wie die Ergebnisse der Matched-Pair Analyse von geförderten und nicht geförderten Betrieben in der FhG-ISI Befragung Innovationen in der Produktion zeigen, konnte das Rahmenkonzept die Forschungsanstrengungen der geförderten Unternehmen statistisch signifikant erhöhen. Bei der Berechung der FuE-Aufwendungen wurden für die geförderten Unternehmen die die im jeweiligen Jahr erhaltene Fördersumme von den FuE Aufwendungen abgezogen, um so reine Additionalitäten ausweisen zu können. Auch auf mögliche Selektionseffekte (z.B. Wissensintensität der Betriebe, Förderung bereits im Vorläuferprogramm) wurde kontrolliert.

So investieren beispielsweise geförderte Unternehmen mit im Mittel 8,1 Mio. EUR einen deutlich höheren Betrag in FuE, als das die nicht geförderten Zwillinge mit im Durchschnitt 3,7 Mio. EUR tun. Entsprechend ist auch die FuE-Intensität der geförderten Betriebe nach Erhalt der Förderung mit $9 \%$ signifikant höher als ihren nicht geförderten Pendants, die etwa 7 \% FuE-Anteil am Umsatz aufweisen. Ähnliche Befunde zeigen sich für das FuE Personal als Indikator für die intern erbrachte FuE: Auch hier weisen geförderte Unternehmen mit im Mittel etwa 88 MitarbeiterInnen im Bereich FuE einen deutlich höheren Wert aus als die nicht geförderten Vergleichsunternehmen (49 MitarbeiterInnen) und haben dementsprechend auch einen signifikant höheren Anteil des FuE-Personals am Gesamtpersonal (16\% gegenüber 
$13 \%)$. Insgesamt führt die Förderung im Rahmenkonzept also zu einer deutlichen Steigerung des Innovationsinputs. Diese positiven Additionalitätseffekte sind für alle untersuchten Indikatoren statistisch auf dem $10 \%$-Niveau signifikant.

Da die Zielgruppe der kleinen und mittleren Unternehmen (KMU) für die Verbundvorhaben des Rahmenkonzepts „Forschung für die Produktion von morgen“ besondere Relevanz besitzt, haben wir die FuE-Additionalitäten für diese Gruppe gesondert berechnet und ausgewiesen (Abbildung 13). Wie sich zeigt, lassen sich auch für die Gruppe der KMU signifikante Unterschiede zwischen geförderten und nicht geförderten Unternehmen bei drei der vier verwendeten Indikatoren nachweisen. Lediglich bei der absoluten Anzahl des FuE-Personals ist der Unterschied zwar durchaus merklich, aber statistisch nicht signifikant. So weisen die geförderten KMU mit durchschnittlich 1,1 Mio. EUR signifikant höhere FuE-Aufwendungen aus als ihre vergleichbaren ungeförderten Kontrollunternehmen mit etwa 0,8 Mio. EUR. Folglich ist die FuE-Intensität der geförderten KMU mit durchschnittlich etwa $11 \%$ ebenfalls merklich höher als bei den ungeförderten Pendants mit etwa $8 \%$. Auch der Anteil der FuE MitarbeiterInnen am Gesamtpersonal ist mit $18 \%$ in den geförderten KMU signifikant höher als in der entsprechenden Vergleichsgruppe (13\%). Insgesamt kann also auch für die geförderten KMU im Rahmenkonzept konstatiert werden, dass die Förderung zu deutlich positiven Input-Effekten bei den FuEAktivitäten der Unternehmen führt.

\section{Abbildung 13 FuE Additionalitäten der Förderung für alle Unternehmen und für die Teilgruppe der KMU}

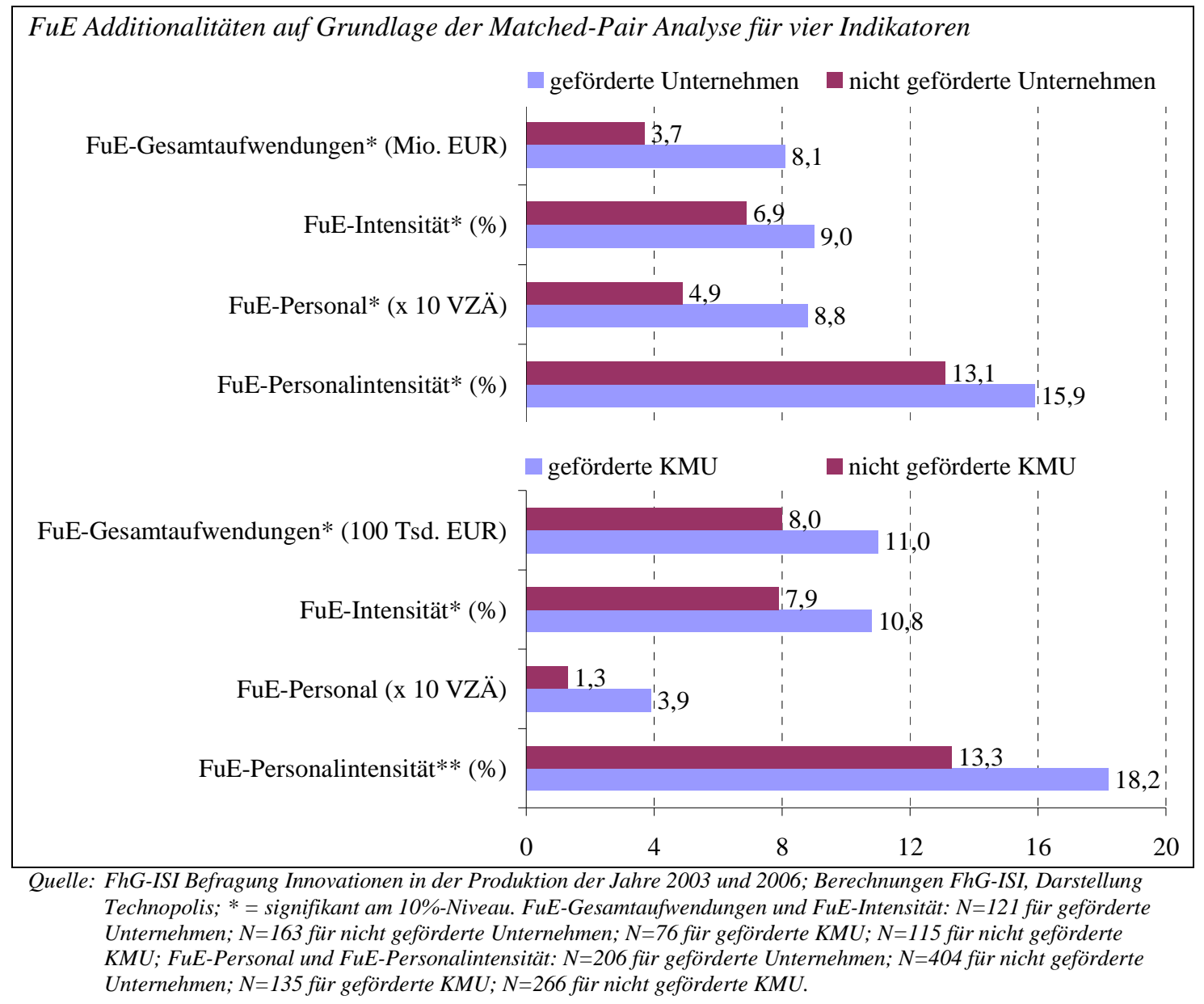




\subsection{Wirtschaftliche Verwertung der Projektergebnisse}

Mit der Erhebung der wirtschaftlichen Wirkungen bei den beteiligten Unternehmen sind eine Reihe von methodischen und praktischen Problemen verbunden. Erstens, kann eine kurzfristige, unmittelbare Auswirkung der Projektergebnisse auf den Umsatz oder die Kosten im Bereich der vorwettbewerblichen FuE nicht erwartet werden. Mit einer wirtschaftlichen Verwertung und Auswirkungen auf Umsatz, Kosten und Erträge ist in größerem Umfang erst einige Jahre nach Projektabschluss zu rechnen. Die geförderten FuE-Vorhaben sind üblicherweise auch Teil eines Bündels von Maßnahmen im betrieblichen Innovationsprozess. Wir haben in dieser Evaluation mit einem Matched-Pair Ansatz versucht, diese Probleme soweit als möglich zu umschiffen und können hier belastbare Ergebnisse über den Umfang der wirtschaftlichen Verwertung und den mit der Förderung erzielten Effekt auch im Vergleich mit im ähnlichen nicht geförderten Unternehmen präsentieren.

\section{Einführung von Produktinnovationen und produktbegleitenden Dienstleistungen}

Um die Effekte der Förderung im Bereich Produktinnovation abzubilden, haben wir in der Matched-Pair Analyse einen Vergleich zwischen geförderten und nicht geförderten Unternehmen durchgeführt, die zwischen 2003 und 2005 Produktneuheiten bzw. Marktneuheiten auf den Markt bringen konnten. Nach dem Oslo Manual der OECD werden unter Produktneuheiten Produkte verstanden, die weniger als drei Jahre im Markt und neu für das Unternehmen sind. Marktneuheiten sind dagegen Produkte, die jünger als drei Jahre sind und nicht nur für das Unternehmen, sondern auch für den Markt neu sind.

Wie die Ergebnisse zeigen, ist der Anteil der Unternehmen, die Produkt- oder Marktneuheiten im Betrachtungszeitraum auf den Markt gebracht haben, bei den geförderten Unternehmen signifikant höher als bei den ungeförderten Zwillingen. Dies gilt sowohl für die Beobachtung aller Unternehmen wie auch die Subgruppe der KMU. So haben jeweils etwa $80 \%$ der geförderten Unternehmen im Beobachtungszeitraum für jeweils ihr Unternehmen neue Produkte auf den Markt gebracht, während die entsprechende Quote bei den ungeförderten Betrieben nur bei jeweils etwa $70 \%$ liegt. Ein ähnliches Bild zeigt sich für den Anteil der Unternehmen, die im Betrachtungszeitraum auch für den Markt neue Produkte erfolgreich eingeführt haben: hier liegt der Anteil bei den geförderten Unternehmen bei über $50 \%$ und ist damit signifikant höher als in der Gruppe der nicht geförderten Kontrollunternehmen mit etwa $40 \%$. Keine signifikanten Unterschiede zeigen sich dagegen beim Umsatzanteil, der im Bilanzjahr 2005 mit Produktinnovationen bzw. mit Marktneuheiten generiert werden konnte.

Die analysierten Effekte sind nicht auf unterschiedliche Innovations-Performance vor dem Förderzeitpunkt zurückzuführen. Da das Matching auch auf die vergangene Innovations-Performance, gemessen am Umsatzanteil von Produkten, die zwischen drei und zehn Jahre alt sind, kontrolliert, wird hier mit hoher Wahrscheinlichkeit nicht die Selektion innovativerer Unternehmen gemessen, sondern tatsächlich die Wirkung der Förderung. Insgesamt zeigen die Ergebnisse, dass die Förderung in der Lage ist, die Unternehmen dabei zu unterstützen, mehr Produktneuheiten und Marktneuheiten zu entwickeln. Jeweils etwa $10 \%$-Punkte mehr geförderte Unternehmen, die im Vergleich zu ihren ungeförderten Pendants Produkt- oder Marktinnovatoren sind, sprechen eine deutliche Sprache. In einem Förderprogramm, das vorwett- 
bewerbliche Verbundprojektforschung als zentrales Instrument nutzt, kann nicht mehr erwartet werden, als dass die Quote der Produkt- und Marktinnovatoren bei den geförderten Unternehmen merklich positiv beeinflusst wird. Die beteiligten Unternehmen haben also bereits relativ kurz nach Projektende Produkt- oder Marktinnovationen entwickeln und einführen können.

\section{Abbildung 14 Wirkung der Förderung bei Produktinnovationen}

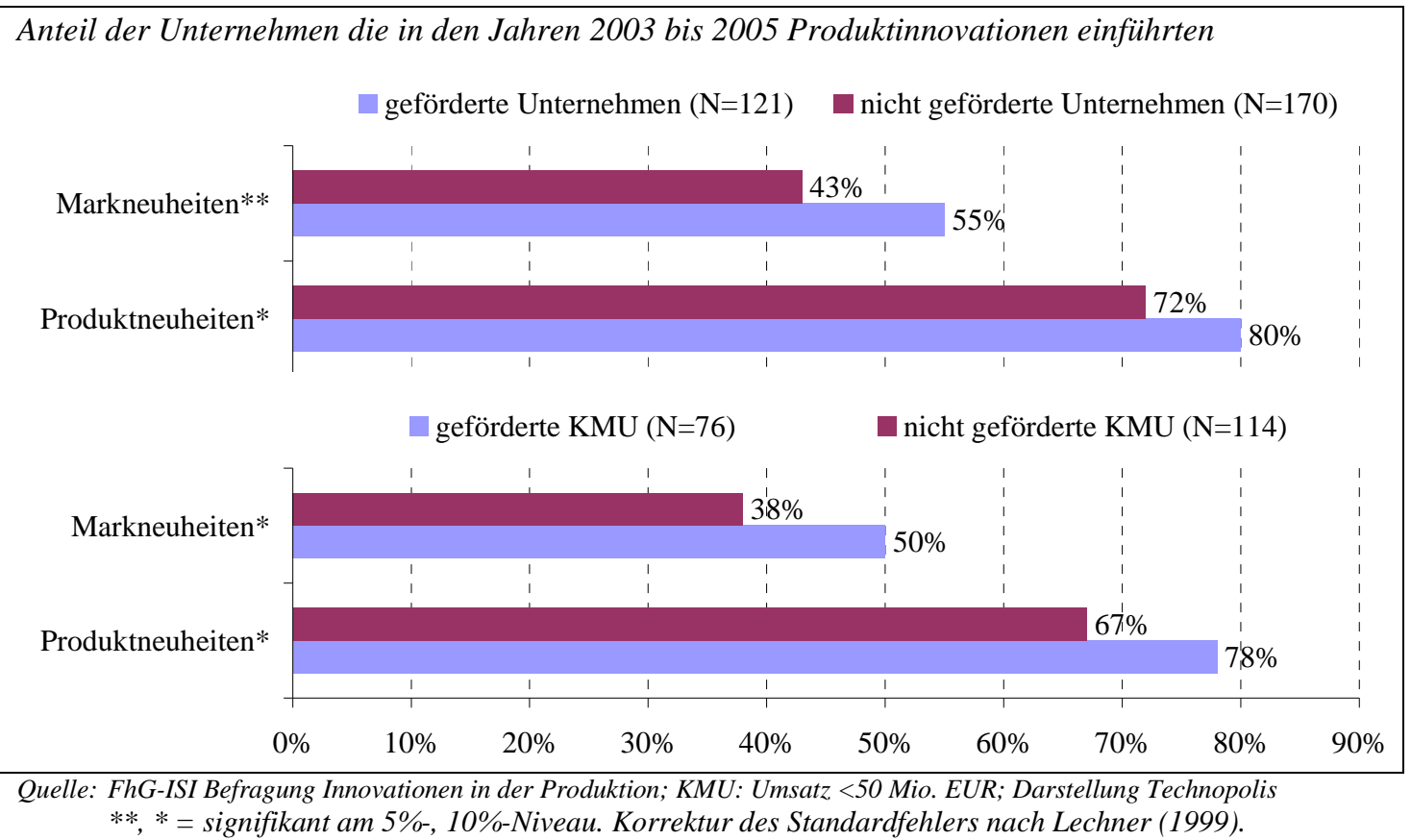

Ähnliche Effekte der Projektförderung können wir auch für den Bereich der produktbegleitenden Dienstleistungen feststellen. Dabei unterscheiden wir in der Analyse die Unternehmen danach, ob sie in den drei Jahren von 2003 bis 2005 ihren Kunden erstmalig bestimmte Dienstleistungen angeboten haben. Die Ergebnisse zeigen, dass bei den geförderten Unternehmen die Tendenz produktbegleitende Dienstleistungen neu in das eigene Leistungsangebot aufzunehmen und Kunden anzubieten, bei fast allen Indikatoren höher ist als bei vergleichbaren nicht geförderten Unternehmen. Dies gilt insbesondere für die Dienstleistungen SoftwareErstellung, Montage und Inbetriebnahme des Produkts beim Kunden, Schulung sowie Wartung und Reparatur inklusive Teleservice. Betrachten wir nur die Gruppe der KMU, sehen wir signifikante Unterschiede zwischen geförderten und nicht geförderten Unternehmen insbesondere im Angebot innovativer Leasing, Vermietungs- und Finanzierungsdienstleistungen für den Kunden.

Generell führt die Förderung dazu, dass die geförderten Unternehmen nicht nur einzelne Dienstleistungen gezielt stärker anbieten, sondern insbesondere ein breiteres Sortiment an komplementären Dienstleistungen anbieten und erbringen. Wie auch bei den Produktinnovationen zuvor zeigt sich noch keine nachweisbare Wirkung auf den kommerzialisierbaren Umsatzanteil, der mit diesen für die Unternehmen neuen Dienstleistungen erwirtschaftet werden konnte. Auch bei Dienstleistungsinnovation ist also wie bei Produkt- und Marktneuheiten mit einer erheblichen Zeitverzögerung zwischen der Einführung der Dienstleistungsinnovation und messbaren zusätzlichen Umsatzanteilen zu rechnen. 
Tabelle 8 Effekte der Förderung bei produktbegleitenden Dienstleistungen

\begin{tabular}{|c|c|c|c|c|}
\hline \multirow{3}{*}{$\begin{array}{l}\text { Art der produktbegleitenden } \\
\text { Dienstleistungsinnovation }\end{array}$} & \multicolumn{2}{|c|}{ Alle Unternehmen } & \multicolumn{2}{|c|}{ KMU } \\
\hline & keine & & keine & \\
\hline & Förderung & Förderung & Förderung & Förderung \\
\hline Planung, Beratung, Projektierung & $\begin{array}{c}4 \% \\
(\mathrm{~N}=394)\end{array}$ & $\begin{array}{c}7 \% \\
(\mathrm{~N}=197)\end{array}$ & $\begin{array}{c}3 \% \\
(\mathrm{~N}=206)\end{array}$ & $\begin{array}{c}7 \% \\
(\mathrm{~N}=103)\end{array}$ \\
\hline Dokumentation & $\begin{array}{c}3 \% \\
(\mathrm{~N}=394)\end{array}$ & $\begin{array}{c}4 \% \\
(\mathrm{~N}=197)\end{array}$ & $\begin{array}{c}3 \% \\
(\mathrm{~N}=206)\end{array}$ & $\begin{array}{c}4 \% \\
(\mathrm{~N}=103)\end{array}$ \\
\hline Software & $\begin{array}{c}1 \% \\
(\mathrm{~N}=394)\end{array}$ & $\begin{array}{l}\text { 6\%**** } \\
(\mathrm{N}=197)\end{array}$ & $\begin{array}{c}5 \% \\
(\mathrm{~N}=206)\end{array}$ & $\begin{array}{c}7 \% \\
(\mathrm{~N}=103)\end{array}$ \\
\hline Leasing, Vermietung, Finanzierung & $\begin{array}{c}6 \% \\
(\mathrm{~N}=394)\end{array}$ & $\begin{array}{c}6 \% \\
(\mathrm{~N}=197)\end{array}$ & $\begin{array}{c}2 \% \\
(\mathrm{~N}=206)\end{array}$ & $\begin{array}{c}\mathbf{7 \%} * \\
(\mathrm{~N}=103)\end{array}$ \\
\hline Montage, Inbetriebnahmen & $\begin{array}{c}1 \% \\
(\mathrm{~N}=394)\end{array}$ & $\begin{array}{c}4 \% * * \\
(\mathrm{~N}=197)\end{array}$ & $\begin{array}{c}3 \% \\
(\mathrm{~N}=206)\end{array}$ & $\begin{array}{c}5 \% \\
(\mathrm{~N}=103)\end{array}$ \\
\hline Schulung & $\begin{array}{c}1 \% \\
(\mathrm{~N}=394)\end{array}$ & $\begin{array}{c}6 \% * * \\
(\mathrm{~N}=197)\end{array}$ & $\begin{array}{c}5 \% \\
(\mathrm{~N}=206)\end{array}$ & $\begin{array}{c}6 \% \\
(\mathrm{~N}=103)\end{array}$ \\
\hline Wartung, Reparatur & $\begin{array}{c}1 \% \\
(\mathrm{~N}=394)\end{array}$ & $\begin{array}{c}4 \% * \\
(\mathrm{~N}=197)\end{array}$ & $\begin{array}{c}2 \% \\
(\mathrm{~N}=206)\end{array}$ & $\begin{array}{c}5 \% \\
(\mathrm{~N}=103)\end{array}$ \\
\hline Betrieb & $\begin{array}{c}2 \% \\
(\mathrm{~N}=394)\end{array}$ & $\begin{array}{c}3 \% \\
(\mathrm{~N}=197)\end{array}$ & $\begin{array}{c}3 \% \\
(\mathrm{~N}=206)\end{array}$ & $\begin{array}{c}3 \% \\
(\mathrm{~N}=103)\end{array}$ \\
\hline $\begin{array}{l}\text { Umsatzanteil produktbegleitender } \\
\text { Dienstleistungen (bis } 3 \text { Jahre alt) }\end{array}$ & $\begin{array}{c}2,2 \\
(\mathrm{~N}=394)\end{array}$ & $\begin{array}{c}2,7 \\
(\mathrm{~N}=197)\end{array}$ & $\begin{array}{c}3,3 \\
(\mathrm{~N}=206)\end{array}$ & $\begin{array}{c}3,2 \\
(\mathrm{~N}=103)\end{array}$ \\
\hline
\end{tabular}

\section{Umsätze mit Produkten und Dienstleistungen}

In der Teilnehmerbefragung haben wir die Industriepartner ebenfalls nach der wirtschaftlichen Verwertung der Ergebnisse der geförderten FuE-Vorhaben gefragt. Wir fragten dabei nach Umsätzen mit Produkten und Dienstleistungen, die unmittelbar auf den Projektergebnissen aufbauen. Insgesamt 99 Industriepartner berichteten in der Befragung über Umsätze, die direkt auf den Projektergebnissen aufbauen.

Dies entspricht einem Anteil von 32\% der Industriepartner, deren FuE-Vorhaben zum Zeitpunkt der Befragung bereits abgeschlossen war. Darunter befinden sich 78 Industriepartner (25\%) mit Umsätzen mit Produkten und 48 Industriepartner (15\%) mit Umsätzen mit Dienstleistungen.

In der folgenden Tabelle fassen wir die Angaben der Industriepartner zur Höhe der heute bereits erzielten (jährlichen) Umsätze zusammen. Bei der Ermittlung dieser Werte aus den Angaben der Befragten sind wir so vorgegangen, dass für jeden einzelnen Fall die Plausibilität der Höhe der Umsätze über einen Vergleich mit dem Gesamtumsatz kontrolliert wurde.

Der Median-Wert (d.h. die Hälfte der Antwortenden berichten Umsätze unter bzw. über diesem Wert) liegt für Umsätze mit Produkten bei 250.000 EUR pro Jahr, für Umsätze mit Dienstleistungen bei 80.000 EUR pro Jahr. Die Summe der derzeit erzielten Umsätze mit Produkten und Dienstleistungen der Industriepartner, die auf den Vorhabensergebnissen aufbauen, beträgt heute bereits etwa das Eineinhalbfache der aufgewandten Fördermittel für alle Industriepartner bzw. das etwa das Sechsfache der aufgewandten Fördermittel der Industriepartner, die Umsätze berichteten. 
Tabelle 9 Umsätze mit Produkten und Dienstleistungen, die unmittelbar auf den Vorhabensergebnissen aufbauen

\begin{tabular}{|c|c|c|c|}
\hline Umsätze & Produkte & Dienstleistungen & $\begin{array}{c}\text { Produkte und } \\
\text { Dienstleistungen }\end{array}$ \\
\hline $\begin{array}{l}\text { Anzahl Industriepartner: } \\
\text { von diesen berichten ... }\end{array}$ & 78 & 48 & 99 \\
\hline $25 \%$ jährliche Umsätze bis zu ... & 72.500 EUR & 21.000 EUR & 50.000 EUR \\
\hline $50 \%$ jährliche Umsätze bis zu ... & 250.000 EUR & 80.000 EUR & 250.000 EUR \\
\hline $75 \%$ jährliche Umsätze bis zu ... & 1.000.000 EUR & 300.000 EUR & 1.000.000 EUR \\
\hline $90 \%$ jährliche Umsätze bis zu ... & 5.100.000 EUR & 1.000.000 EUR & 5.000.000 EUR \\
\hline berichteter Gesamtumsatz: & 113.041.000 EUR & 15.158.000 EUR & 128.199.000 EUR \\
\hline $\begin{array}{l}\text { Umsatz in \% der Gesamtförder- } \\
\text { mittel für alle Industriepartner } \\
\text { in \% der Gesamtfördermittel der }\end{array}$ & & & $154 \%$ \\
\hline Industriepartner mit Umsätzen & & & $618 \%$ \\
\hline
\end{tabular}

Bei den Institutspartnern spielen Umsätze mit Produkten wenig überraschend eine vergleichsweise geringe Rolle, dafür haben Umsätze mit Dienstleistungen relativ betrachtet eine größere Bedeutung. Nur neun der 130 Institutspartner deren FuEVorhaben bereits abgeschlossen waren (7\%) berichteten über Umsätze mit Produkten, jedoch 46 Institute (35\%) über Umsätze mit Dienstleistungen. Ingesamt berichteten 48 Institute über Umsätze als unmittelbares Resultat der Vorhabensergebnisse (37\%). Hier möchten wir allerdings festhalten, dass bei den beteiligten Instituten die wirtschaftliche Verwertung der Projektergebnisse sicherlich nicht als wesentlichste Erfolgsdimension im Programm gesehen werden kann. Die Verwertung soll in erster Linie in den geförderten und nicht geförderten Betrieben stattfinden.

Der Anteil an Industriepartnern, der die Ergebnisse des Verbundprojekts unmittelbar in Form von Produkten und Dienstleistungen vermarkten kann (etwa ein Drittel aller Industriepartner) erscheint uns im Lichte eines ambitionierten FuE-Programms mit Verbundprojekten, die ein zum Teil hohes inhaltliches Projektrisiko aufweisen, als überaus erfolgreich.

\section{Einführung von technischen Prozessinnovationen}

Die Matched-Pair Analyse erlaubt es uns auch, Aussagen darüber zu machen, wie sich geförderte und nicht geförderte Unternehmen hinsichtlich der Einführung von technischen Prozessinnovationen im Zeitraum 2003 bis 2005 unterscheiden.

Erfahrung mit den abgefragten Produktionstechniken vor dem Jahr 2003 wurden beim Matching berücksichtigt, so dass sichergestellt ist, dass tatsächlich Effekte der Förderung und nicht Selektivitäten gemessen werden.

Über die berücksichtigten Prozesstechniken hinweg stellen wir durchgängig eine Tendenz zur verstärkten Implementierung von technischen Prozessinnovationen in geförderten Unternehmen fest. Statistisch signifikant ist der Unterschied allerdings nur die Einführung von Virtual Reality bzw. 3D-Simulation zur Produktkonzeption. Doch auch bei der Nutzung von Bildverarbeitungstechniken, Simulationsmodellen zur Prozessauslegung oder dem Austausch von Dispositionsdaten zur Unterstützung des Supply Chain Management zeigen sich durchaus beträchtliche Unterschiede zwischen geförderten Unternehmen und nicht geförderten Unternehmen. 


\section{Abbildung 15 Effekte der Förderung bei technischen Prozessinnovationen}

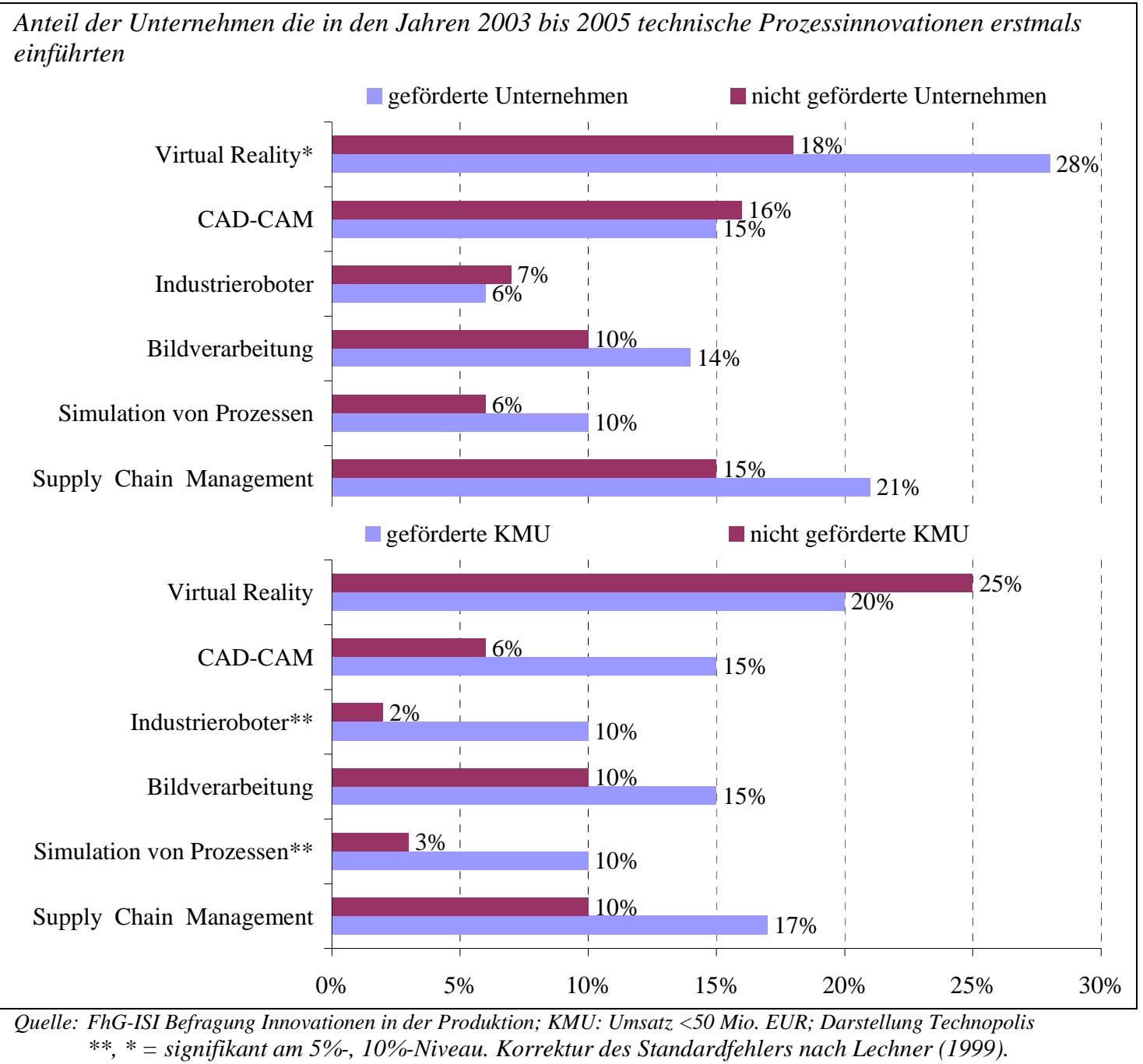

Für die Gruppe der KMU zeigen sich deutliche und statistisch signifikante Effekte bei der Implementierung von Industrierobotern bzw. Handhabungstechniken und der Nutzung von Simulationen zur optimierten Prozessauslegung. Die signifikant positiven Effekte liegen sowohl bei den geförderten KMU wie auch bei allen geförderten Unternehmen rund 8-10 \%-Punkte über der Einführungsquote dieser Techniken bei nicht geförderten Unternehmen. Die beobachteten Differenzen sind angesichts des kurzen Betrachtungszeitraums von drei Jahren zwischen 2003 und 2005 durchaus bemerkenswert. Insgesamt scheint demnach die Projektförderung die technologische Wettbewerbsfähigkeit in den Produktionsprozessen der beteiligten Unternehmen tatsächlich positiv zu beeinflussen.

\section{Einführung von organisatorischen Innovationen}

Analog zum Vergleich der Einführung von technischen Prozessinnovationen haben wir auch für verschiedene innovative Organisationskonzepte untersucht, ob sich im Betrachtungszeitraum 2003 bis 2005 die Anteile jener Unternehmen, die bestimmte Konzepte neu einführten, zwischen geförderten bzw. nicht geförderten Unternehmen unterscheiden. Vorhandene Vorerfahrungen mit innovativen organisatorischen Prozessen wurden beim Matching der Unternehmen berücksichtigt. 
Die Ergebnisse zeigen, das sich für zwei der fünf untersuchten Organisationskonzepte positive Effekte der Förderung nachweisen lassen. So weisen im Vergleich die geförderten Unternehmen bei der Implementierung von Just-in-time und Simultaneous Engineering Konzepten signifikant höhere Einführungsquoten auf. Von den geförderten Unternehmen führten in den Jahren 2003 bis 2005 erstmalig 14\% kundenorientierte Just-in-time-Prinzipien und 12 \% Simultaneous EngineeringKonzepte ein, während die jeweiligen Anteile bei den ungeförderten Unternehmen lediglich bei $7 \%$ bzw. $4 \%$ lagen. Wenn wir in der Auswertung nur KMU betrachten stellen wir bei drei der fünf untersuchten innovativen Organisationskonzepten höhere Einführungsquoten bei den geförderten KMU fest, allerdings sind die Unterschiede zu den nicht geförderten KMU statistisch nicht signifikant.

\section{Abbildung 16 Effekte der Förderung bei organisatorischen Innovationen}

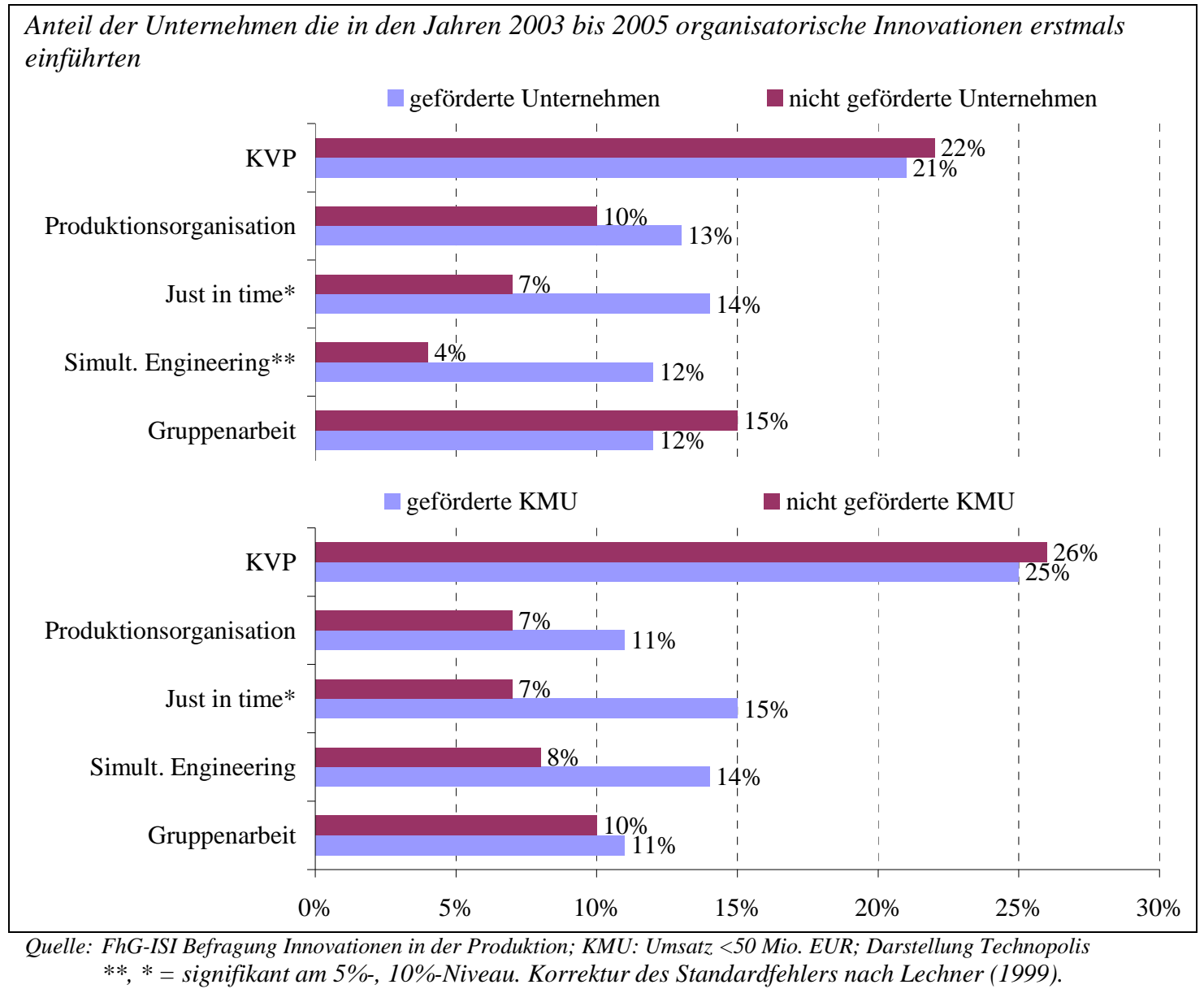

Lediglich bei den abgefragten arbeitsorganisatorischen Konzepten kontinuierlicher Verbesserungsprozess (KVP) und Einführung der Gruppenarbeit in der Produktion sehen wir keine großen Unterschiede zwischen geförderten und nicht-geförderten Unternehmen, während die Effekte bei Indikatoren Produktionsorganisation, Just in time und Simultaneous Engineering beträchtlich sind. Möglicherweise spiegeln sich in diesen Ergebnissen auch Förderschwerpunkte in den nicht technisch orientierten Themen der Handlungsfeldern Marktorientierung und strategische Produktplanung (HF1), Neue Formen der Zusammenarbeit produzierender Unternehmen (HF3) und Der Mensch und das wandlungsfähige Unternehmen (HF4) wider. Die Ergebnisse interpretieren wir jedenfalls als ein Indiz für die Wirksamkeit der Förderung weicher Verbundprojekte im Rahmenkonzept. In den beteiligten Unternehmen werden 
tatsächlich organisatorische Wandlungsprozesse angestoßen, die zu einer besseren Positionierung gegenüber Kunden führen. Damit wird mit der Förderung ein (messbarer) Beitrag zur Verbesserung der Wettbewerbsposition des deutschen Produktionssektors geleistet.

\section{Kostensenkungen und Ertragszuwachs}

Hinsichtlich der Effekte auf die Kosten und die Ertragslage im Betrieb gaben in Summe 40 der 267 antwortenden Betriebe (15\%) an, dass mit den Ergebnissen des FuE-Vorhabens unmittelbar die Kosten im Betrieb verringert werden konnten (siehe Abbildung 17). Insgesamt 74 von 260 antwortenden Industriepartnern (28\%) gaben an, dass sich die Ertragsstärke des Betriebs durch die Ergebnisse der FuE-Vorhaben verbessert hätte. Von diesen Partnern quantifizierten 54 Antwortende das Ausmaß des Ertragszuwachses. Die angegebenen Werte reichen von 0,2\% bis 100\% Ertragszuwachs. Höhere Erträge durch die Anwendung der Projektergebnisse in den Unternehmen werden also nicht nur durch geringere Kosten realisiert, sondern scheinen in signifikantem Ausmaß einen Einfluss auf wachstumsrelevante Faktoren zu haben.

\section{Abbildung 17 Einfluss der Projektergebnisse auf Kosten und Ertragsstärke}

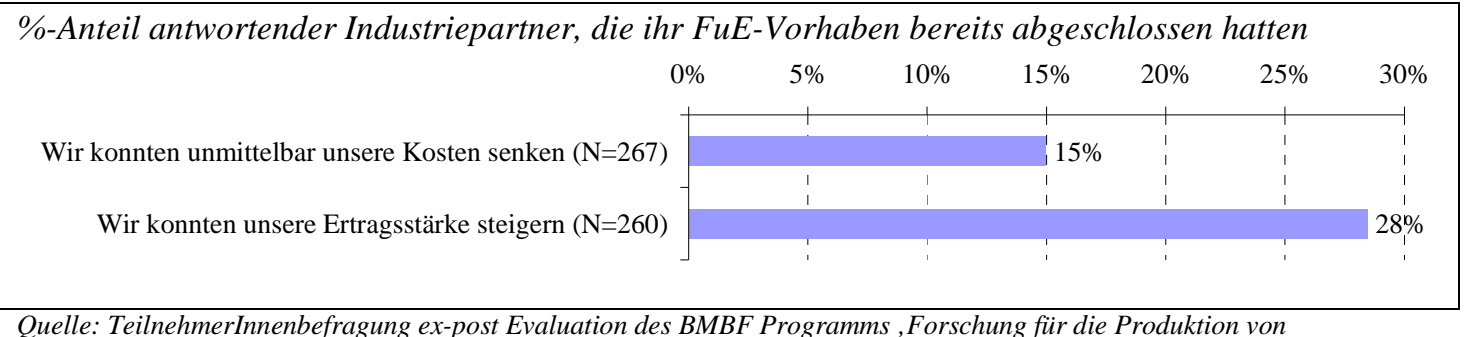

Quelle: TeilnehmerInnenbefragung ex-post Evaluation des BMBF Programms, Forschung für die Produktion von morgen' für die Jahre 1999 bis 2004; Berechnungen und Darstellung Technopolis

\section{Patentierung und Vergabe von Lizenzen}

Insgesamt gaben 35 von 300 (12\%) der auf diese Frage antwortenden Industriepartner an, Ergebnisse der Verbundprojekte patentiert zu haben. Lizenzen für Entwicklungen aus den Verbundprojekten wurden fünf Industriepartnern vergeben. Dies entspricht rund 2\% der antwortenden Teilnehmer aus der Industrie. Nicht in der obigen Graphik dargestellt sind die Angaben der Institutspartner. Hier gaben 17 von 126 antwortenden Instituten an (14\%), Ergebnisse patentiert zu haben. Zwei Institute berichteten über eine aktuelle Verwertung durch Lizenzen.

\section{Abbildung 18 Patentierung und Lizenzierung von Projektergebnissen}

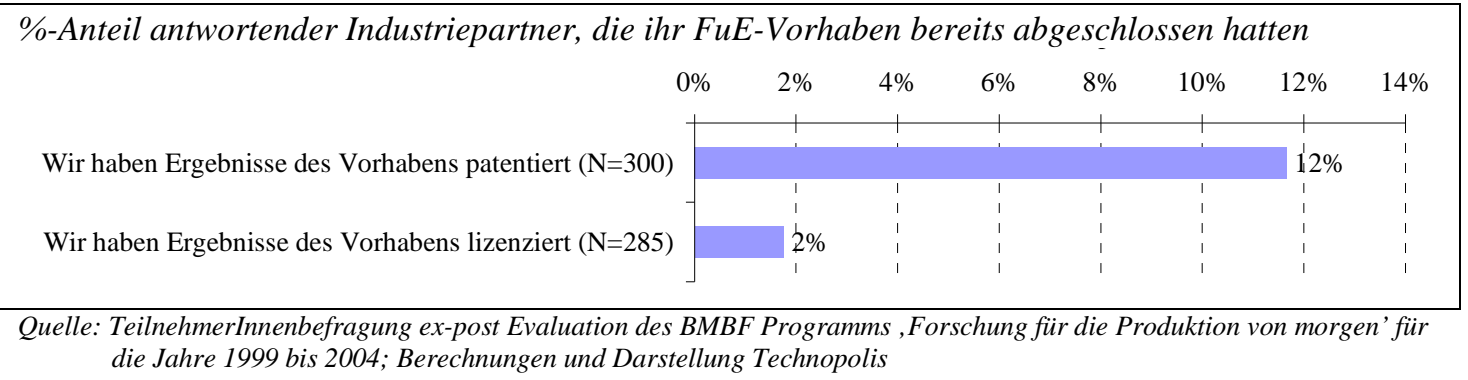




\subsection{Wirkung der Projektteilnahme auf die Kompetenzen der Betriebe und auf die Bewältigung von Innovationshemmnissen}

Welche organisatorischen und wirtschaftlichen Wirkungen hatten die Projektergebnisse in den teilnehmenden Organisationen? In welchem Ausmaß haben die Projekte dazu beigetragen, interne und externe Innovationshemmnisse wahrzunehmen bzw. zu überwinden? Die Industriepartner wurden dazu danach befragt, welche Auswirkungen die Teilnahme am Verbundprojekt auf ihren Märkten (Abbildung 19) und im Betrieb (Abbildung 20) nach sich zogen.

\section{Abbildung 19 Projektwirkungen auf den Märkten}

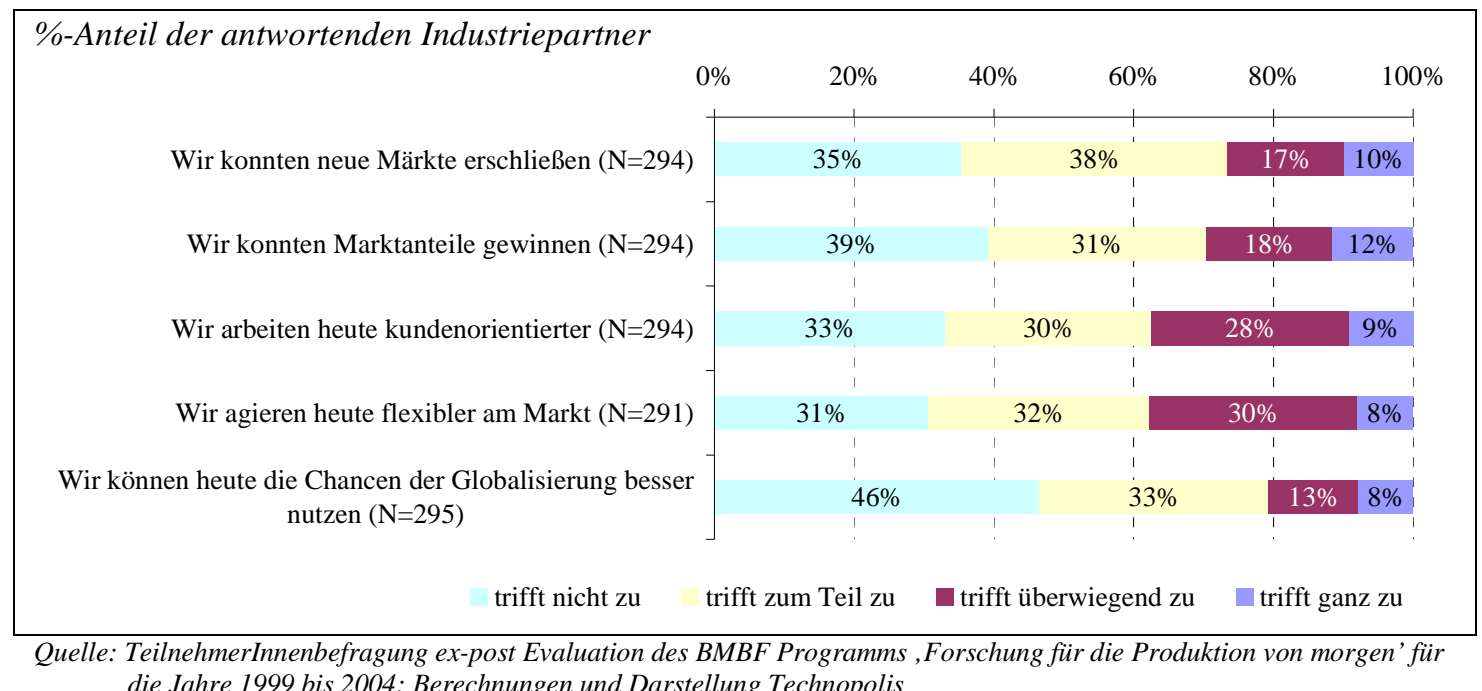

Den größten positiven Effekt (gemessen als Anteil der Antworten mit ,trifft ganz zu' und ,trifft überwiegend zu') hatten die Projekte in den Betrieben auf die Faktoren ,Flexibilität am Markt' und ,Kundenorientierung'. Insgesamt 38\% der Betriebe gab an als Resultat der Projektteilnahme heute auf den Märkten flexibler zu agieren und $37 \%$ heute kundenorientierter zu arbeiten. Mehr als ein Viertel (27\%) der Betriebe sah eine positive Wirkung auf die Erschließung neuer Märkte und 30\% gaben an, einen positiven Effekt auf die Gewinnung von Marktanteilen feststellen zu können.

Den relativ betrachtet geringsten Beitrag auf den Märkten leisteten die FuE-Vorhaben nach Einschätzung der TeilnehmerInnen bezüglich der besseren Nutzung von Globalisierungschancen. Nur etwas mehr als jeder fünfte Industriepartner (21\%) konnte der Aussagen ganz oder überwiegend zustimmen, dass durch die Projektteilnahme heute die Chancen der Globalisierung besser genutzt werden können.

Das Ziel, MitarbeiterInnen in den Betrieben in Hinblick auf die künftigen Herausforderungen in der Produktion besser zu qualifizieren scheint durch die BMBF Projektförderung im großen und ganzen erreicht worden zu sein. Die Hälfte der Industriepartner (50\%) gab an, einen relevanten positiven Effekt auf die Qualifizierung der MitarbeiterInnen im Betrieb feststellen zu können (gemessen als Anteil der Antworten mit, trifft ganz zu' und ,trifft überwiegend zu'). In $48 \%$ der Fälle trug die Projektteilnahme dazu bei, dass die Betriebe heute besser in Kooperationsnetzwerke eingebettet sind. Insgesamt 45\% der Industriepartner gab an, dass die Projekteilnahme zur Sicherung bzw. Schaffung von Arbeitsplätzen in Deutschland beigetragen hat. Vier von zehn Industriepartner (40\%) gaben an, dass aufgrund der Projektteil- 
nahme das Management heute mehr Verständnis für die Bedeutung von FuE zeigt und fast einem Drittel der Betriebe (30\%) fällt es heute leichter, FuE im Betrieb zu finanzieren.

\section{Abbildung 20 Projektwirkungen im Betrieb}

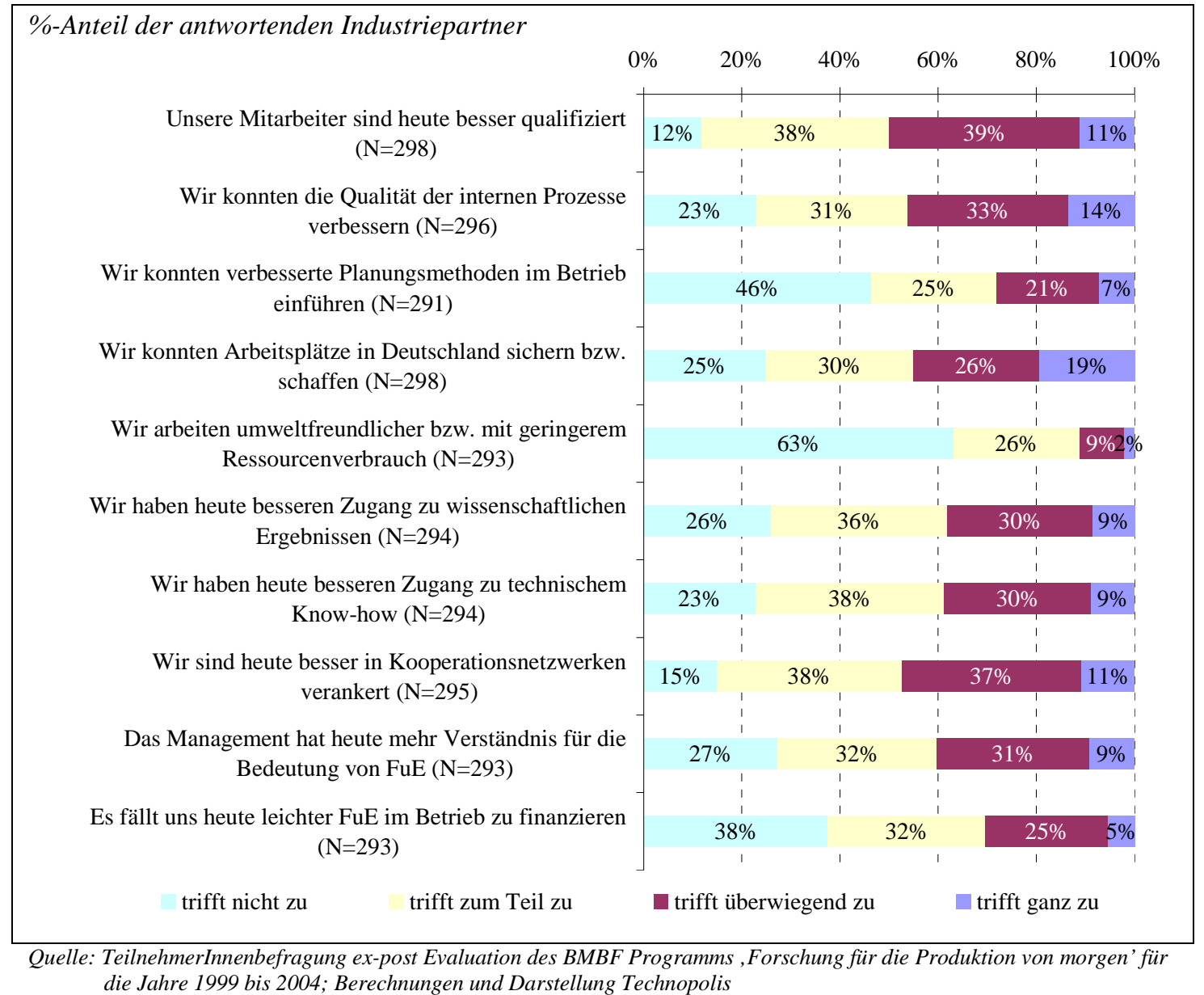

Die Forschungsförderung des BMBF im Rahmenkonzept ,Forschung für die Produktion von morgen' hat damit nach Einschätzung der Antwortenden in einem beträchtlichem Ausmaß zur Bewältigung von internen und externen Innovationshemmnissen (z.B. Zugang zu Wissen und Know-how, Zugang zu Finanzierungsquellen, Verfügbarkeit von qualifiziertem Personal) geführt, und auch einen Beitrag zur nachhaltigen Sicherung von Beschäftigung in der Produktion in Deutschland leisten können.

Den mit Abstand geringsten Effekt hatte die BMBF Projektförderung auf ,Umweltschutz und Ressourceneinsparung' in den teilnehmenden Betrieben. Nur etwas mehr als jeder neunte Betriebe (11\%) gab an, durch die Projektteilnahme einen relevanten positiven Effekt feststellen zu können. Auch wenn man nur Betriebe des Verarbeitenden Gewerbes berücksichtigt (da für viele Dienstleister im Programm diese Wirkungsdimension im eigenen Betrieb nicht unbedingt relevant sein dürfte), steigt der Anteil jener Industriepartner, die hier einen positiven Effekt beobachten konnten, nur unwesentlich an. 


\section{Box 3: Fallstudie ChopArc: Technologietransfer über Köpfe}

Wann können wir davon sprechen, dass ein Verbundprojekt wirtschaftlich erfolgreich war? Die Fallstudie des Verbundprojekts ChopArc macht zweierlei deutlich. Erstens: zu Ende des Verbundprojekts ist eine abschließende Antwort über den Projekterfolg und die wirtschaftliche Verwertung der Ergebnisse (und Fehlschläge) des Projekts nicht möglich. Und zweitens: der Hauptnutznießer der wirtschaftlichen Verwertung muss nicht immer aus dem Kreis der Projektpartner kommen.

Ziel von ChopArc war die Entwicklung eines neuartigen MAG Lichtbogen Schweißverfahrens, mit dem auch Blechdicken unterhalb 0,5 mm Dicke wirtschaftlich miteinander verbunden werden können. Ökonomisch interessant ist diese Technologie besonders für die Automobilindustrie und für Anwendungen im Leichtbau.

Organisatorisch verlief das Verbundprojekt turbulent. Der am Projekt beteiligte Schweißanlagenhersteller traf relativ früh im Projekt die Entscheidung sich aus der Entwicklung automatisierter Schweißanlagen zurückzuziehen. Damit war die Entwicklung des benötigten Demonstrators für den Anwender aus der Automobilbereich gefährdet. Aber auch der Anwender selbst schied bald aus dem Projekt aus, da im Unternehmen schließlich auf ein anderes Verfahren zum automatischen Schweißen dünner Bleche gesetzt wurde. Damit stand das Projekt kurz vor dem Platzen: Es gab keinen Anwender, der die Industrieanforderungen an das zu entwickelnde Verfahren definieren und zuliefern hätte können.

Über Kontakte des beteiligten Universitätsinstituts im Deutscher Verband für Schweißen und verwandte Verfahren e.V. (DVS) konnte schließlich ein neuer Anwender für das Projekt gewonnen werden. Allerdings änderten sich die Eigentumsverhältnisse dieses Unternehmens kurze Zeit nach Projekteintritt. Die mit der Eingliederung in die Muttergesellschaft verbundene Neuordnung der Fertigung an den verschiedenen Produktionsstandorten führte zu erheblichen Verzögerungen im Projektverlauf.

Auch technisch traf das Projekt auf zahlreiche Hindernisse. Die wissenschaftlichen Vorarbeiten dauerten länger als geplant und die für die vorgesehenen Messungen notwendige Stromquelle stand erst verspätet zur Verfügung. Die zahlreichen Probleme und Verzögerungen im Projekt führten praktisch dazu, dass der als Projektergebnis angestrebte Demonstrator im Rahmen des Projekts nicht fertig gestellt werden konnte. Erst später wurde der Demonstrator in Kooperation mit einem nicht geförderten Schweißanlagenhersteller fertig entwickelt. Den größten wirtschaftlichen Nutzen zu Projektende hatte das beteiligte Softwareunternehmen, das die ChopArc Module erfolgreich in seine Standardprodukte integrierte und für andere industriellen Anwendungen zur 3D-Strömungsberechnung vermarkten konnte.

Insgesamt müsste man zu diesem Zeitpunkt mit Blick auf die wirtschaftliche Verwertung der Ergebnisse von einem gescheiterten Verbundprojekt sprechen. Wäre da nicht die Person des wissenschaftlichen Projektkoordinators. Er verlässt kurze Zeit nach Abschluss von ChopArc gemeinsam mit einem Projektmitarbeiter die Universität und baut bei seinem neuen Arbeitgeber EWM, einem nicht am Projekt beteiligten Hersteller von Schweißgeräten, auf den Erfolgen und Misserfolgen von ChopArc auf. Es gelingt ihm in dem neuen Unternehmen, ein adaptiertes Verfahren zur Marktreife zu bringen, mit dem das wärmereduzierte Fügen dünnster Bleche möglich wird. Erstmalig lassen sich auch Mischverbindungen wie Aluminium-Stahl, Magnesium-Stahl und Aluminium-Magnesium verarbeiten. Das neue Verfahren wurde inzwischen mit dem ABICOR-Innovationspreis 2006 des DVS ausgezeichnet. 


\section{Wirkung der Projektteilnahme auf die Kompetenzen der Betriebe und auf die Bewältigung von Innovationshemmnissen nach Betriebsgröße}

Für die weiteren Auswertungen haben wir die oben beschriebenen Wirkungen der Projektteilnahme auf den Märkten und im Betrieb mittels Faktoranalyse zu drei Hauptwirkungsdimensionen zusammengefasst: organisatorische Kompetenz gestärkt; Forschungskompetenz gestärkt und Marktkompetenz gestärkt.

Wenn wir die Antworten der Befragten nach der Betriebsgröße differenzieren, so zeigt sich, dass es Mittelbetrieben (50 bis 250 Beschäftigte) am ehesten gelungen ist, ihre organisatorische Kompetenz durch die Projektteilnahme zu erhöhen. In Großbetrieben (über 1.000 Beschäftigte) ist dieser Effekt am geringsten. Dies ist möglicherweise dadurch erklärbar, dass in großen Betrieben mit einem breiterem FuE-Portfolio der Einfluss eines einzelnen Projekts auf die Organisation geringer ausfällt als in kleineren Unternehmen.

\section{Abbildung 21 Wirkungen der Projektteilnahme nach Betriebsgröße}

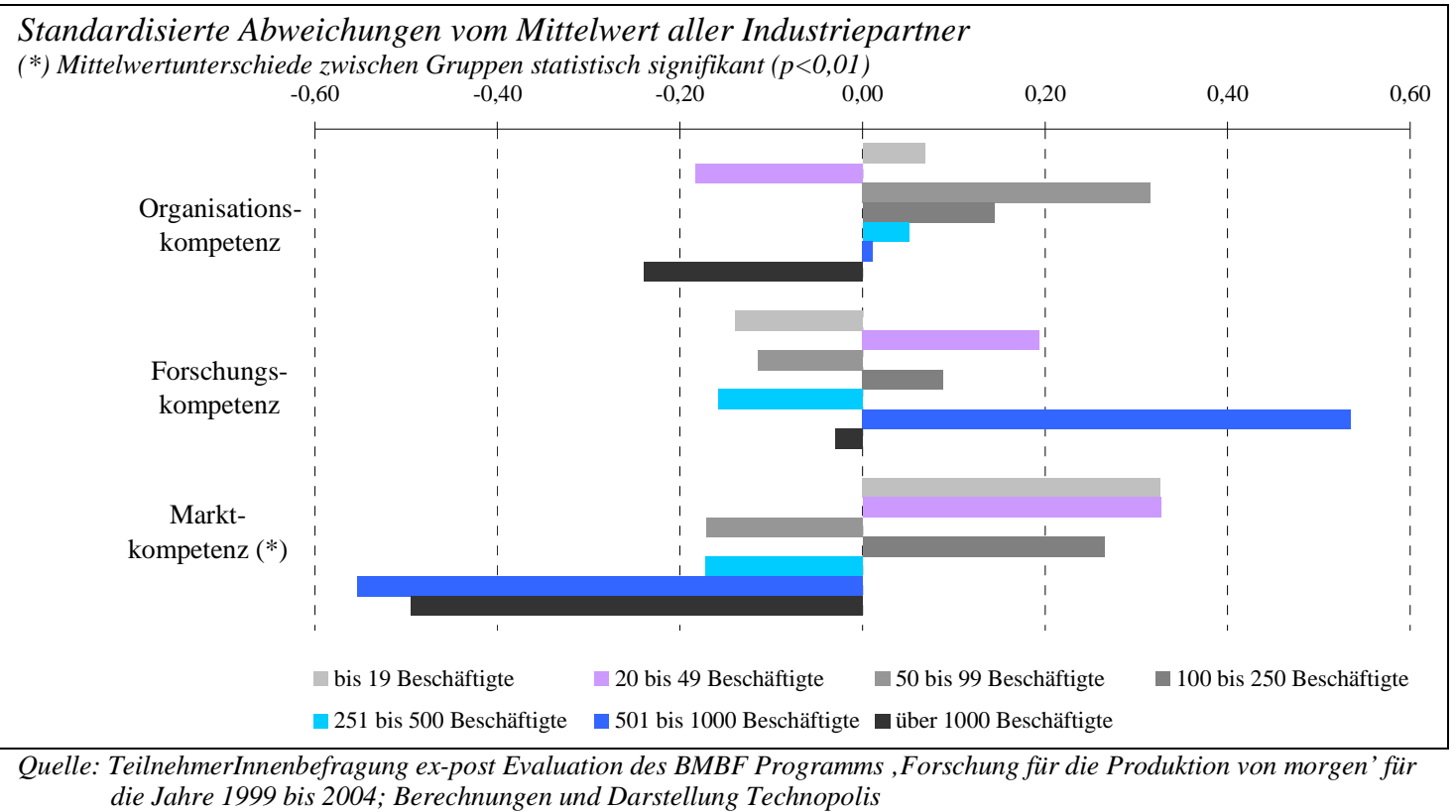

Bezüglich der Stärkung der Forschungskompetenz zeigt sich, dass hier Betriebe in der Größenklasse 501 bis 1.000 Beschäftigte von der Projektteilnahme überproportional profitieren konnten. Eine mögliche Interpretation für dieses Ergebnis ist, dass in Betrieben dieser Größe bereits die notwendigen FuE-Strukturen, Kapazitäten sowie die notwendige Aufmerksamkeit des Managements vorhanden sind, um nachhaltig externes Wissen und Know-how aufnehmen zu können. In kleineren Betrieben sind diese Voraussetzungen tendenziell weniger anzutreffen. Andererseits, kann in Großbetrieben über 1.000 Beschäftigte ein bereits bestehendes Innovationsbewusstsein mit stabilen FuE-Routinen vorausgesetzt werden, das durch ein einzelnes Projekt üblicher weise nicht signifikant beeinflusst wird.

Bei der Hauptwirkungsdimension Marktkompetenz gestärkt zeigt sich, dass kleinere Betriebe die Projektergebnisse in signifikant größerem Ausmaß in eine verbesserte Marktposition umsetzen konnten - von der Projektteilnahme also unmittelbar wirtschaftlich stärker profitierten - als größere Unternehmen. Besonders Betriebe mit 
bis zu 50 Beschäftigten und solche mit 100 bis 250 Beschäftigten konnten durch die Teilnahme am Verbundprojekt ihre Marktkompetenz überproportional ausbauen.

\section{Wirkung der Projektteilnahme auf die Kompetenzen der Betriebe und auf die Bewältigung von Innovationshemmnissen nach Innovationsgrad der Vorhaben}

In einem weiteren Schritt haben wir analysiert, inwieweit der Innovationsgrad des FuE-Projekts in Zusammenhang mit den erzielten Wirkungen am Markt und im Betrieb gebracht werden kann. Dazu haben wir einen Vergleich der Mittelwerte der Hauptwirkungsdimensionen getrennt nach inhaltlichen Projektzielen für die Industriepartner durchgeführt (siehe Abbildung 22).

\section{Abbildung 22 Wirkungen der Projektteilnahme nach Innovationsgrad}

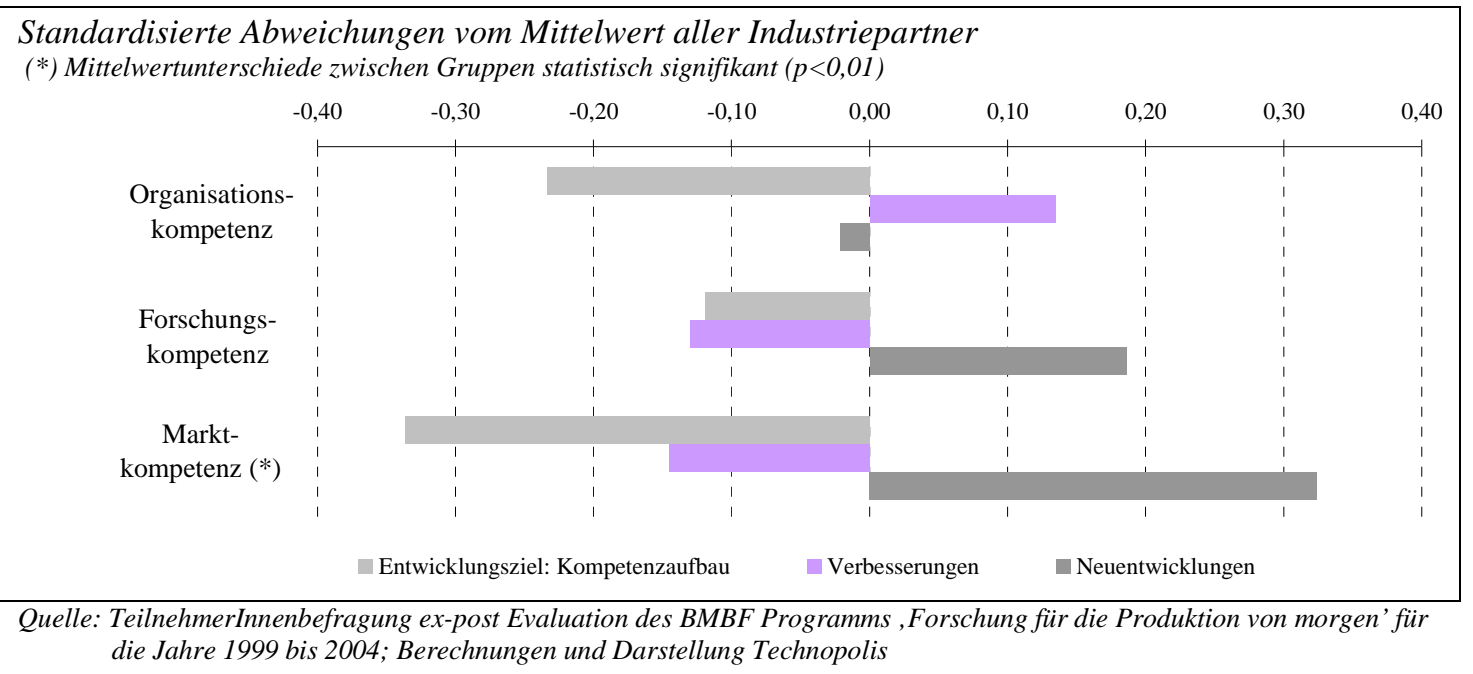

Es zeigt sich, dass Betriebe, deren primäres Ziel es war, Erfahrungen und Kompetenz aufzubauen bzw. Grundlagenentwicklung zu betreiben, in allen drei Wirkungsdimensionen nur unterdurchschnittlich erfolgreich waren. Dieses Ergebnis kann dahingehend interpretiert werden, dass Erfahrungsaustausch und Kompetenzaufbau, losgelöst von einem konkreten Entwicklungs- und Verwertungsinteresse in den Betrieben, nur vergleichsweise geringe Wirkungen nach sich ziehen. Bei der Wirkungsdimension, Organisationskompetenz gestärkt' waren vor allem jene Betriebe überdurchschnittlich erfolgreich, die Entwicklungen anstrebten, die bereits auf bestehende Produkte und Dienstleistungen aufsetzen. Bei diesen FuE-Vorhaben ist der positive Beitrag zu verbesserten internen Prozessen, der Weiterqualifizierung der MitarbeiterInnen sowie zur stärkeren Kundenorientierung besonders ausgeprägt. Industriepartner, deren Ziel es war, völlig neue technisch, organisatorisch und strategisch-orientierte Entwicklungen in Angriff zu nehmen haben überdurchschnittlich ihre FuE-Kompetenz ausweiten können und waren auch bei der Erschließung neuer Märkte und der Sicherung von Beschäftigung in Deutschland am erfolgreichsten. Diese Ergebnisse lassen den Schluss zu, dass bei einer stärkeren Orientierung der Förderung auf Projekten die auf die Entwicklung neuer Lösungen abzielen, nicht mit Abstrichen beim Ausmaß der Erreichung der technischen, organisatorischen oder strategischen Ziele zu rechnen ist, sondern, dass gerade solche Vorhaben bezüglich ihrer Wirkungen auf die Kompetenzen der Betriebe besonders positiv einzuschätzen sind. 


\subsection{Hemmnisse beim Transfer von Projektergebnissen}

Welche Faktoren sind dafür verantwortlich, dass Ergebnisse bzw. entwickelte Lösungen aus den Verbundprojekten nicht wirtschaftlich verwendet werden? Abbildung 23 gibt einen Überblick über die Antworten der Industriepartner in der Teilnehmerbefragung zu möglichen Gründen.

Die wichtigsten Barrieren für die Anwendung der Ergebnisse liegen im technischen Bereich: Fast vier von zehn Industriepartnern (38\%), die ihr Projekt bereits abgeschlossen hatten, gaben an, dass die entwickelten Lösungen technisch noch nicht ausgereift sind; in $22 \%$ der Fälle sind die Lösungen noch nicht leistungsfähig genug. An zweiter Stelle stehen Marktfaktoren als Barrieren für die Anwendung: 20\% der Industriebetriebe gaben an, für die entwickelten Lösungen bestehe keine Marktnachfrage und in etwa einem Fünftel der Fälle (19\%) sahen die Antwortenden die Wirtschaftlichkeit der Lösungen nicht als gegeben. Mangelnde technische Kompatibilität mit den betrieblichen Rahmenbedingungen (12\%) und geänderte Prioritäten (11\%) folgen als Gründe, warum der Einsatz der Vorhabensergebnisse nicht möglich erscheint. Inhaltliches Scheitern der FuE-Vorhaben spielt als Grund, warum es zu keiner Anwendung der Projektergebnisse kommt eine geringe Rolle. Nur 9\% der Industriepartner, die ihr Vorhaben bereits abgeschlossen hatten, gaben an, dass das Projekt keine verwertbaren Ergebnisse lieferte. Diese Zahlen machen deutlich, dass auch nach dem Abschluss der FuE-Vorhaben in den Verbundprojekten noch ein beträchtlicher technischer Entwicklungsbedarf notwendig ist.

\section{Abbildung 23 Gründe, warum Ergebnisse nicht angewendet werden}

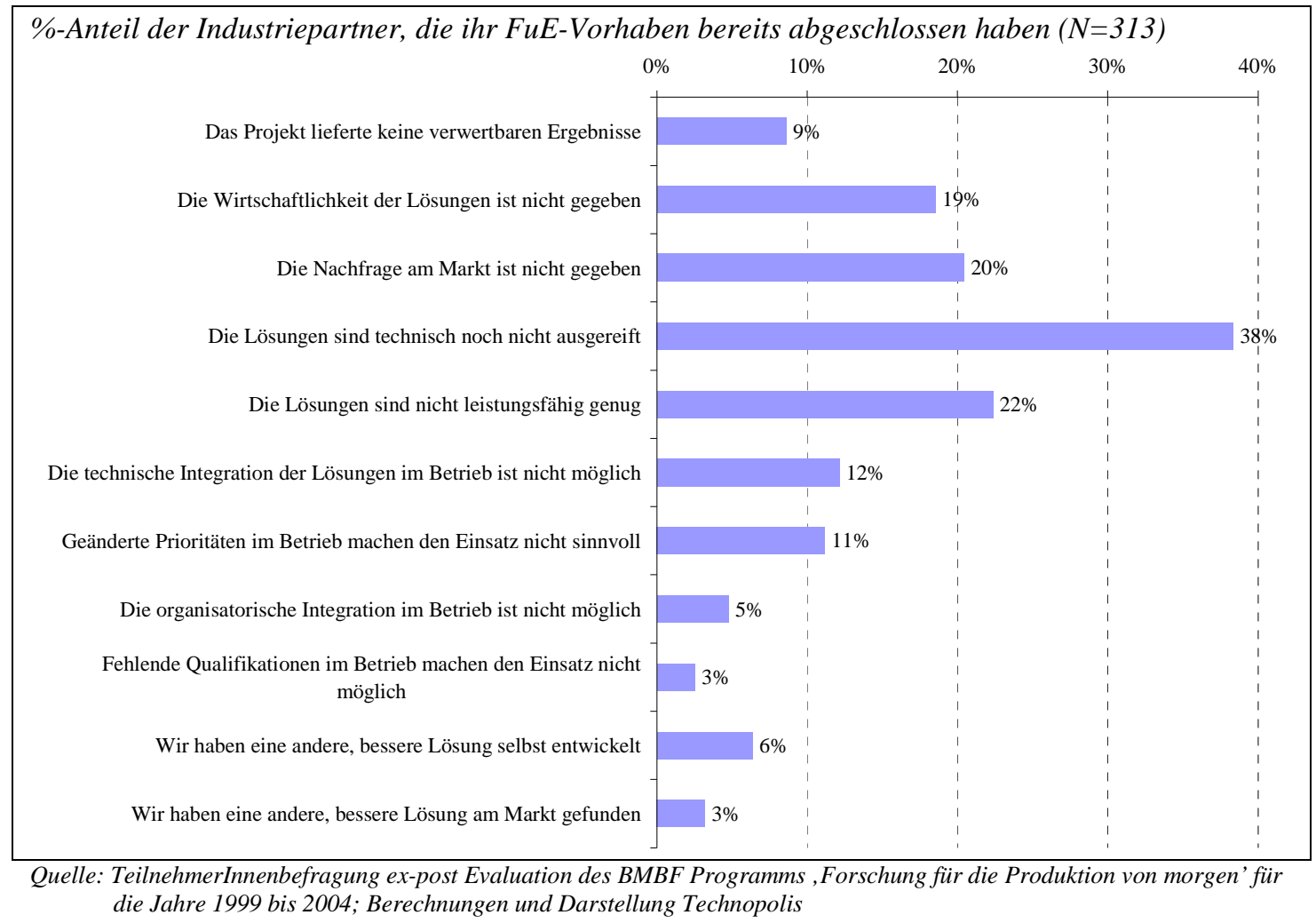


In diesem Zusammenhang interessierte uns auch die Frage, ob die Einbeziehung von Anwendern in die Verbundprojekte die Wahrnehmung von Gründen, die eine Anwendung von Lösungen behindern, beeinflusst. Dazu haben wir für Industriepartner mit Rolle Entwickler oder Zulieferer, die ihr FuE-Vorhaben bereits abgeschlossen hatten, die obige Auswertung gesondert durchgeführt, wobei wir zwischen FuEVorhaben mit bzw. ohne Einbeziehung von Anwendern unterschieden haben.

Auch wenn die für die Auswertung zur Verfügung stehenden Fälle vergleichsweise gering sind (siehe Tabelle 10) deuten die Ergebnisse doch darauf hin, dass Entwickler und Zulieferer in Verbundprojekten, in denen Anwender einbezogen wurden, tendenziell sensibler für die Wahrnehmung von Gründen sind, die eine Anwendung der entwickelten Ergebnisse und Lösungen behindern: 27 von 59 Entwicklern und Zulieferer in Verbundprojekten mit Anwendern (46\%) fanden, dass die Lösungen noch nicht leistungsfähig genug seien, verglichen mit nur 12 von 51 Entwicklern und Zulieferer in Verbundprojekten ohne Anwender (24\%). Auch sahen 21 von 59 Entwicklern und Zulieferer in Verbundprojekten mit Anwendern (36\%) die Nachfrage am Markt nicht gegeben, im Vergleich zu 11 von 50 Entwicklern und Zulieferer in Verbundprojekten ohne Anwender (22\%). Die Einbeziehung von Anwendern in die Verbundprojekte scheint also den Blick der Entwickler und Zulieferer für die Probleme der Nutzung der Projektergebnisse zu schärfen.

\section{Tabelle 10 Gründe, warum Entwickler und Zulieferer Ergebnisse der FuE-Vorhaben nicht anwenden}

\begin{tabular}{|c|c|c|c|c|c|c|}
\hline \multirow[b]{3}{*}{ Gründe } & \multicolumn{3}{|c|}{$\begin{array}{l}\text { Verbundprojekte } \\
\text { ohne Anwender }\end{array}$} & \multicolumn{3}{|c|}{$\begin{array}{l}\text { Verbundprojekte } \\
\text { mit Anwendern }\end{array}$} \\
\hline & & trifft & in & & trifftt & in \\
\hline & $\mathbf{N}$ & $\mathbf{z u}$ & $\%$ & $\mathbf{N}$ & $\mathbf{z u}$ & $\%$ \\
\hline Das Projekt lieferte keine verwertbaren Ergebnisse & 51 & 4 & $8 \%$ & 59 & 11 & $19 \%$ \\
\hline Die Wirtschaftlichkeit der Lösungen ist nicht gegeben & 49 & 12 & $24 \%$ & 59 & 16 & $27 \%$ \\
\hline Die Nachfrage am Markt ist nicht gegeben & 50 & 11 & $22 \%$ & 59 & 21 & $36 \%$ \\
\hline Die Lösungen sind technisch noch nicht ausgereift & 51 & 23 & $45 \%$ & 61 & 36 & $59 \%$ \\
\hline Die Lösungen sind nicht leistungsfähig genug & 51 & 12 & $24 \%$ & 59 & 27 & $46 \%$ \\
\hline $\begin{array}{l}\text { Die technische Integration der Lösungen im Betrieb } \\
\text { ist nicht möglich }\end{array}$ & 49 & 3 & $6 \%$ & 58 & 7 & $12 \%$ \\
\hline Geänderte Prioritäten machen den Einsatz nicht sinnvoll & 47 & 5 & $11 \%$ & 57 & 12 & $21 \%$ \\
\hline $\begin{array}{l}\text { Die organisatorische Integration im Betrieb ist nicht möglich } \\
\text { Fehlende Qualifikationen im Betrieb machen den Einsatz }\end{array}$ & 47 & 1 & $2 \%$ & 57 & 4 & $7 \%$ \\
\hline nicht möglich & 47 & 1 & $2 \%$ & 57 & 3 & $5 \%$ \\
\hline Wir haben eine andere, bessere Lösung selbst entwickelt & 48 & 3 & $6 \%$ & 56 & 3 & $5 \%$ \\
\hline Wir haben eine andere, bessere Lösung am Markt gefunden & 48 & 1 & $2 \%$ & 56 & 1 & $2 \%$ \\
\hline
\end{tabular}




\section{Box 4: Fallstudie OPAQ: Technische und organisatorische Innovationen gehen Hand in Hand}

Neue technologische Lösungen zu entwickeln ist eine Sache. Diese dann erfolgreich in die vorhandenen Prozesse und organisatorischen Abläufe zu integrieren die zumeist schwierigere - andere. Die Fallstudie des Verbundprojekts OPAQ zeigt, wie durch eine enge Kooperation zwischen Technologieentwickler und Anwender marktfähige Lösungen möglich werden. Die Fallstudie macht zudem deutlich, dass gemeinsame Forschungsprojekte Unternehmenskulturen und organisatorische Abläufe ändern können. Die Wirkungen von Forschungsprojekten strahlen damit weit über die erzielten technischen Ergebnisse aus.

Ziel von OPAQ war es, ein optisches System für das Erkennen und die Bewertung von Oberflächenfehlern nach Umformprozessen zu entwickeln. Das Messsystem sollte Unregelmäßigkeiten in Oberflächen von Freiformteilen schon ab 10 Mikrometer erkennen. In Bezug auf Indikatoren wie Fehler-Erfassungszeit und Zuverlässigkeit wäre ein solches System dem menschlichen Auge weit überlegen.

Als Entwickler des Inspektionssystems arbeiteten ein Fraunhofer-Institut mit einem Softwareunternehmen und Steinbichler Optotechnik, einem Anbieter von optischen Sensoren und Messtechnik-Produkten, zusammen. Auf Anwenderseite beteiligten sich AUDI sowie die Seidel GmbH \& Co., ein Unternehmen mit Schwerpunkt Oberflächenveredelung und Fertigung von Aluminium-Umformteilen für die kosmetische und pharmazeutische Industrie, am Projekt.

Nach Ende des Projekts entwickelte Steinbichler Optotechnik die Sensoren und Softwareapplikationen in Zusammenarbeit mit AUDI bis zur Serienreife weiter. Die Firma vermarktet heute das System weltweit erfolgreich in der Automobil- und Luftfahrtindustrie. Rund zehn neue Arbeitsplätze konnten dadurch im Unternehmen geschaffen werden. Beim Anwender AUDI führt das neue System zu geringeren Kosten, da Fertigungsfehler beim Umformen früher erkannt werden. Die größte Herausforderung für AUDI bei der Einführung war, die organisatorische Integration des neuen Inspektionssystems im Presswerk zu meistern. Inzwischen wird bereits daran gedacht, das System auch in anderen Abteilungen, wie dem Karosseriebau, der Lackiererei oder dem Werkzeugbau einzusetzen.

Bei Seidel GmbH \& Co. lässt sich der wirtschaftliche Projekterfolg heute nicht am praktischen Einsatz des Verfahrens ablesen. Die enorme Teilevielfalt bei Kappen und Verschlüssen hat zur Konsequenz, dass unterschiedlichste Fehler auftreten können. Dem Unternehmen erschienen die Risiken der notwendigen Weiterentwicklung des Systems für eine erfolgreiche technische und organisatorische Integration in die Produktion als zu hoch. Trotzdem hat OPAQ die Routinen bei Seidel nachhaltig verändert: auf technischer Ebene beschleunigte die Teilnahme an OPAQ die Nutzung von modernen Bildverarbeitungsverfahren für Anwendungen in der Qualitätssicherung. Es wurden inzwischen - im Vergleich zu OPAQ technisch weniger anspruchsvolle optische Systeme für die Oberflächeninspektion von Kleinteilen erfolgreich installiert.

Geändert hat sich auch Vieles im nicht-technischen Bereich: OPAQ gab für das Unternehmen den Anstoß für eine Professionalisierung des Forschungsmanagements und den Einstieg in andere ambitionierte Forschungsvorhaben. Das Qualifikationsniveau im Unternehmen stieg: Es werden heute mehr HochschulabsolventInnen beschäftigt. Nicht zuletzt hat sich die Zusammenarbeit und das gegenseitige Verständnis von Fachkräften und Akademikern durch OPAQ verbessert. 


\subsection{Anmerkungen zu Wirkungen im ungeförderten Bereich}

Die von uns durchgeführten Arbeiten im Rahmen der Matched-Pair Analyse und der Teilnehmerbefragung können methodisch nur die Effekte in den geförderten Unternehmen selbst statistisch analysieren und erklären. Ob die in den Verbundprojekten entwickelten und in Pilotvorhaben bzw. Demonstratoren erprobten Lösungskonzepte tatsächlich auch, wie förderpolitisch gewünscht, entweder für eine breite Zielgruppe vermarktbar oder in ihren Prinzipien auf eine möglichst große Gruppe anderer Unternehmen übertragbar sind, bleibt damit unbeantwortet.

Die Beantwortung der Frage nach der Wirksamkeit und Breite des Transfers der entwickelten Lösungen in nicht an den Verbundvorhaben beteiligten Betrieben, kann quantitativ-empirisch nur dann erfasst werden, wenn es sich bei den Lösungen um Vorstufen marktfähiger Produkte oder Dienstleistungen handelt, die dann später als Umsatz mit Produkten und Dienstleistungen gemessen werden können. Technische und insbesondere organisatorische Prozessinnovationen verbreiten sich dagegen zumeist nicht über die Verkaufskanäle der geförderten Betriebe, sondern werden von dritten Unternehmen mehr oder weniger selbständig eingeführt und adaptiert. Der Erfolg dieses horizontalen Transfers kann mit breitenempirischen Befragungen üblicherweise nicht gemessen werden. Hiezu sind qualitative Analysen des Ergebnistransfers nach Projektabschluss sowie der in Frage kommenden Transferkanäle notwendig, wie sie im Rahmen dieser Evaluierung vor allem in den drei durchgeführten Fallstudien durchgeführt wurden (siehe Box 3 bis Box 5).

Bei der Analyse des Transfer- bzw. Kommerzialisierungserfolgs der entwickelten Lösungen gilt es schließlich zu beachten, dass sich dieser üblicherweise mit einer deutlichen Zeitverzögerung einstellt. Dies gilt sowohl für die Vermarktung innovativer Produkte oder Dienstleistungen als auch für den Transfer prinzipiell übertragbarer und erprobter Gestaltungskonzepte in nicht geförderte Betriebe. Bei den meisten geförderten Verbundprojekten im Rahmenkonzept „Forschung für die Produktion von morgen" erlaubt daher die zeitliche Nähe des Projektabschlusses zur Durchführung der Evaluation keine belastbaren Aussagen über die Verbreitung der Ergebnisse in den ungeförderten Bereich.

Abschließend möchten wir im Zusammenhang mit der Frage des Transfers der Ergebnisse auf Dritte bzw. in den ungeförderten Bereich auf einige Eindrücke hinweisen, die wir bei der Durchsicht der Schluss- bzw. Erfolgskontrollberichte gewonnen haben. Im Schlussbericht werden die geförderten Unternehmen aufgefordert, Angaben über die Übertragbarkeit der Projektergebnisse für Dritte zu machen. Wir übertreiben nicht, wenn wir sagen, dass die Betriebe in vielen Fällen mit dieser Frage nichts anzufangen wissen. Die Betriebe machen häufig deutlich, dass sie unter einer Verbreitung der Ergebnisse in erster Linie die Vermarktung der eigenen Produkte und die Anwendung der erarbeiteten Lösungen im eigenen Unternehmen verstehen. Daneben wird auf die Anwendung der Lösungen in anderen Betrieben des Unternehmens bzw. in Schwestergesellschaften im In- und Ausland hingewiesen. Diese Position, Transfer vor allem einmal als proprietäre Nutzung der Ergebnisse zu verstehen, ist aus Sicht der Betriebe gut nachvollziehbar, hat doch der Zuwendungsempfänger das Recht auf ausschließliche Nutzung des Ergebnisses (im Einklang mit der Kooperationsvereinbarung zwischen den Partnern des Verbundprojekts) und trifft ihn gleichzeitig nach den Allgemeinen Nebenbestimmungen für FuE-Zuwen- 
dungen des BMBF an Unternehmen der gewerblichen Wirtschaft (NKBF 98 Nr. 9.2) eine Ausübungs- bzw. Verwertungspflicht.

Bei der Zusammenarbeit mit Stellen außerhalb des Verbundprojekts konzentrieren sich die Betriebe in erster Linie auf die Mitarbeit in Industriearbeitskreisen sowie auf die Vorstellung der Ergebnisse auf Veranstaltungen der Verbände und bei Messen. Sehr häufig werden wiederum die Nutzung der bestehenden Kontakte zu Lieferanten und Kunden als zentrale Transferschienen angeführt. Auch der Kontakt zu nicht in das Projekt involvierten Universitätsinstitute und Forschungseinrichtungen wird genannt. Schließlich wird der Austausch mit Projektpartnern, die in parallel laufenden Verbundprojekten im Rahmen von „Forschung für die Produktion von morgen“", bzw. in Projekten aus anderen Förderprogrammen, gefördert werden in den Berichten als eine praktizierte Form externer Zusammenarbeit erwähnt.

Das Transfer-Instrument des Projektträgers Industriearbeitskreis wird von den beteiligten Projektpartnern als ein wirksamer und praxisnaher Weg zur Diskussion und Verbreitung von Projektergebnissen geschätzt. Die durchaus vorhandene (und im positiven Sinne hoffentlich eigennützige) Bereitschaft der beteiligten Betriebe, ihre gewonnenen Erfahrungen in den Verbundprojekten mit Dritten zu teilen, drückt sich auch im Interesse aus, über das Projektende hinaus an Industriearbeitskreisen teilzunehmen. Vier Fünftel der Betriebe die in den Berichten dazu Angaben machten, gaben an, zukünftig an Industriearbeitskreisen mitarbeiten zu wollen.

Insgesamt geben die Hinweise der Betriebe auf die Verwertung der Projektergebnisse und ihre Aktivitäten zum Transfer an Dritte den Eindruck einer angemessenen Balance zwischen einzelbetrieblichen Verwertungsinteressen und dem Potenzial für eine breitere Nutzung und Anwendung durch Dritte. Dem Anspruch Breitentransfer zu ermöglichen, kann das Rahmenkonzept aber freilich auf Grund der zur Verfügung stehenden Instrumente nicht gerecht werden. 


\section{Box 5: Fallstudie ErGo: Herausforderungen Anwendung und Ergebnistransfer}

Bei der Beurteilung der Wirkungen der Projekte stellen sich unmittelbar zwei Fragen. Erstens jene nach der nachhaltigen Wirkung der Projektförderung: Was hat sich im Unternehmen geändert, das sich ohne das Projekt nicht geändert hätte? Zweitens, jene nach der Nutzung der Projektergebnisse durch Dritte: Werden die erarbeiteten Lösungen heute durch andere Unternehmen genutzt? Und: Wer stellt sicher, dass interessierte Unternehmen die entwickelten Lösungen tatsächlich aufgreifen können? Besonders bei Vorhaben, bei denen die Ergebnisse a priori physisch nicht „greifbar“ sind, ist letztere Frage von besonderer Bedeutung. Es reicht meist nicht aus, die Ergebnisse, weicher' Projekte in Büchern und Softwaretools grundsätzlich verfügbar zu machen. Häufig braucht es aktive Intermediäre, damit sich innovative Strategien und neue organisatorische Ansätze in der Praxis durchsetzen können.

ErGo verfolgte das Ziel, die Globalisierungsfähigkeit von Fachkräften in KMU zu erhöhen und dafür verallgemeinerbare Ansätze zu entwickeln. Zwei industriesoziologische Institute, fünf Beratungseinrichtungen und ein Transferpartner arbeiteten gemeinsam mit zehn KMU des Maschinenbaus an der Entwicklung und Erprobung von arbeitsorganisatorischen Lösungen und personalwirtschaftlichen Regeln bei der Umsetzung von Globalisierungsstrategien.

Können wir heute, mehr als vier Jahre nach Ende des Projekts, in den beteiligten KMU Hinweise finden, dass ErGo eine nachhaltige Wirkung hatte? Die Antwort dazu lautet eindeutig ja. Im Chemnitzer Unternehmen Sitec wurden durch ErGo umfassende Veränderungen in der Unternehmensorganisation angestoßen: Eine projektorientierte Organisation wurde aufgebaut, Planungsprozesse wurden geändert, Anforderungsprofile an neues Personal wurden überarbeitet und ein neues Entlohnungsmodell wurde eingeführt. Sitec verfügt heute über eine eigenständige Vertriebsstruktur im Ausland und konnte seine Exportquote von 15\% auf 25\% steigern. Das ebenfalls am Projekt beteiligte Unternehmen Haacon Hebetechnik nutzte ErGo für den Aufbau von Vertriebsniederlassungen in Frankreich und Österreich. Im Zuge der Umsetzung dieser Globalisierungsstrategie konnten die Auslandsumsätze massiv gesteigert werden.

Lassen sich Hinweise finden, dass die ErGo Ergebnisse über den Kreis der Projektpartner hinaus genutzt werden? Der Interessensverband Chemnitzer Maschinenbau e.V. (ICM) kam in ErGo die Aufgabe zu ein Transferkonzept zu erstellen. Gemeinsam mit der IHK erstellte ICM eine Globalisierungsbroschüre für KMU, deren erste Auflage inzwischen vergriffen ist. Die ErGo Ergebnisse wurden von ICM zu einer neuen Methode weiterentwickelt, die heute in ungeförderten Beratungsprojekten bei der Unterstützung von Unternehmen zur strategischen Planung von Kooperationen zum Einsatz kommt. Der ICM konnte darüber hinaus zahlreiche geförderte Nachfolgeprojekte zum Thema Globalisierung entwickeln, die maßgeblich zum Umsatzwachstum des Vereins beigetragen haben. Auch die beteiligten Institute und Berater konnten die Ergebnisse in neuen Projekten bzw. für ihre Dienstleistungsangebote nutzen.

Insgesamt werfen aber die Hinweise auf die Breitenwirkung die grundsätzliche Frage auf, wie viel Transfer in und durch Verbundprojekte möglich ist und welche Strategien und Akteure am besten geeignet sind, dieser Aufgabe möglichst effektiv nachzukommen. Von den geförderten Betriebe selbst kann hier kaum ein Transferimpuls ausgehen. Die Forschungs- und Transferpartner bauen auf den Ergebnissen auf und bieten neue Dienstleistungen auch für Dritte an. Trotzdem erscheint der unmittelbare Transfer durch Verbundforschung beschränkt zu sein. 


\section{Administrative Programmdurchführung}

Wichtige Kennzahlen zur Umsetzung des Rahmenkonzepts „Forschung für die Produktion von morgen" haben wir bereits in Abschnitt 5 präsentiert. An dieser Stelle möchten wir noch einige Aspekte der administrativen Programmdurchführung diskutieren. Zuerst beschäftigen wir uns mit dem Auswahlverfahren für die geförderten Verbundprojekte. Dann diskutieren wir die thematische Umsetzung des Rahmenkonzepts in den Bekanntmachungen der Jahre 1999 bis 2004. Anschließend präsentieren wir die Einschätzungen der ProgrammteilnehmerInnen zur Programmumsetzung. Schließlich diskutieren wir die Öffentlichkeitsarbeit und die Transfermaßnahmen des Projektträgers im Lichte der Zielsetzungen des Rahmenkonzepts „Forschung für die Produktion von morgen“.

\subsection{Auswahlverfahren}

Das Verfahren zur Auswahl der Verbundprojekte nach der Veröffentlichung der Bekanntmachung durch das BMBF gliedert sich in die folgenden Schritte, die in den QS-Dokumenten des Projektträgers ausführlich geregelt und beschrieben sind:

1 Eingang und Erfassung der Skizzen beim Projektträger

2 Vorprüfung und -bewertung der Skizzen durch den Projektträger

3 Auswahlgespräch: Bewertung und Selektion durch externe Gutacher

4 Erstellung des Projektrahmenplans und der Vorhabensbeschreibungen durch die Projektpartner für die ausgewählten Skizzen

5 Antragstellung durch den Projektpartner

6 Antragsbearbeitung und -prüfung durch den Projektträger

7 Prüfung und gegebenenfalls Bewilligung durch das BMBF

8 Laufendes Projekt

Mit dem Start des Rahmenkonzepts „Forschung für die Produktion von morgen“ wurden die Spielregeln zur Forschungsförderung im Bereich der Produktionsforschung neu formuliert: die Auswahl der Verbundprojekte erfolgt ausschließlich über den Ideenwettbewerb zu den in den Bekanntmachungen genannten vordringlichen Themen. Damit wurde ein wichtiger Schritt zur Erhöhung der Transparenz in der Forschungsförderung gesetzt. Die hohe Beteiligung der Zielgruppen an den Bekanntmachungen ist ein wichtiges Indiz dafür, dass tatsächlich eine hohe Mobilisierung der Forschungsakteure erreicht werden konnte. Auf Aspekte der strategischen Planung von thematischen Schwerpunkten in den Bekanntmachungen werden wir an späterer Stelle in diesem Bericht noch zurückkommen.

Üblicherweise nominiert der Projektträger für jede Bekanntmachung ein Team von ProjektbetreuerInnen, die für die Abwicklung der Bekanntmachung verantwortlich zeichnen. Die eingehenden Skizzen werden von den BetreuerInnen gesichtet und entsprechend der im Rahmenkonzept und in der jeweiligen Bekanntmachung angeführten allgemeinen und speziellen Bewertungskriterien von den BetreuerInnen gesichtet und nach einem ABC-Schema bewertet. Die Bewertung von Skizzen erfolgt immer durch mehrere MitarbeiterInnen des Projektträgers.

Parallel dazu wird für jede Bekanntmachung eine Gruppe unabhängiger ExpertInnen aus Wirtschaft und Wissenschaft eingerichtet. Diese ExpertInnen sind selbst nicht 
mit Skizzen im Wettbewerb vertreten. Als Vorbereitung für das Auswahlgespräch erhalten die ExpertInnen eine Zusammenfassung der eingegangen Skizzen, die Bewertung der Skizzen durch den Projektträger sowie die vollständigen Skizzen der zum Auswahlgespräch eingeladenen Einreicher. Den ExpertInneen steht es jederzeit frei, zusätzliche Skizzen bzw. Informationen beim Projektträger anzufordern.

Im Auswahlgespräch präsentieren die A-bewerteten Einreicher ihre Skizzen und stehen den ExpertInnen für Fragen über ihre geplanten Vorhaben zu Verfügung. Bei den Präsentation der Skizzen sind alle eingeladenen Einreicher anwesend. Im zweiten Teil des Auswahlgesprächs diskutieren die ExpertInnen mit Unterstützung des Projektträgers und des BMBF im Lichte der Präsentationen die verschiedenen Skizzen und treffen ihre Auswahl unter Berücksichtigung der zur Verfügung stehenden Fördermittel. Dabei ist es auch möglich, dass die ExpertInnen auf Grundlage der schriftlichen Unterlagen die Weiterverfolgung von Skizzen vorschlagen, die nicht im Auswahlgespräch präsentiert wurden. Eine schriftliche Benachrichtigung über die Auswahl bzw. die Nicht-Weiterverfolgung von Skizzen erfolgt durch den Projektträger.

Angesichts der hohen fachlichen Kompetenz des Projektträgers halten wir die Vorgangsweise für richtig, die Vorbewertung der Skizzen für das Auswahlgespräch durch die Betreuer des Projektträgers durchführen zu lassen. Den unabhängigen ExpertInnen im Auswahlgespräch kommt dann die Rolle zu unter den grundsätzlich als förderungswürdig eingestuften Skizzen eine weitere Sichtung und Reihung vorzunehmen. Um für alle in Betracht gezogenen Skizzen die gleichen Voraussetzungen im Verfahren zu schaffen, sollten unserer Ansicht nach aber die weiterverfolgten Skizzen ausschließlich aus dem Kreis der im Auswahlgespräch präsentierten Vorhaben stammen. Dies könnte dadurch sichergestellt werden, dass ein etwas größerer Kreis von Einreichern als bisher üblich zum Auswahlgespräch eingeladen wird. Um der ExpertInnenentscheidung durch die Auswahl des Projektträgers nicht vorzugreifen, sollten die ExpertInnen im Vorfeld des Auswahlgesprächs die Möglichkeit erhalten, die Einladungsliste entsprechend mitzugestalten.

Nach der Auswahl der Skizzen wäre unserer Meinung nach eine begründete schriftliche Rückmeldung entlang der im Rahmenkonzept bzw. in der Bekanntmachung genannten Auswahlkriterien an die Einreicher von nicht-erfolgreichen Skizzen zu empfehlen. Einerseits könnten die Einreicher aus den Rückmeldungen für zukünftige Skizzen und Anträge lernen. Andererseits könnte ein Beitrag dazu geleistet werden, der von Beteiligten vor allem in unseren Interviews artikulierte Skepsis bezüglich der Transparenz und Fairness des Auswahlverfahren wirksam zu begegnen (siehe dazu auch die Ergebnisse des Teilnehmerbefragung im folgenden Abschnitt 7.3). Der damit verbundene zusätzliche Aufwand beim Projektträger sollte sich in Grenzen halten, da der Projektträger bereits jetzt in der Vorbereitung des Auswahlgesprächs die Gründe für die Reihung der Skizzen nach dem ABC-Schema für die unabhängigen ExpertInnen entsprechend zusammenfasst.

Die Verbundpartner der ausgewählten Projektskizzen werden vom Projektträger in einer zweiten Verfahrensstufe aufgefordert, innerhalb von zwei Monaten einen förmlichen Förderantrag mit Projektrahmenplan vorzulegen. Auch eine verbindliche schriftliche Regelung zur Verwertung der Projektergebnisse zwischen den Projektpartnern ist bei Abschluss des Fördervertrags vorzulegen. Der Projektträger berät die 
Verbundpartner bei der Erstellung des Projektrahmenplans und der Förderanträge. Über diese Anträge entscheidet das BMBF nach abschließender Prüfung.

Die weitgehend durchgängige Anwendung des Prinzips „,maximal 50 \% Förderquote über den gesamten Projektverbund" bei der Festlegung der Förderquoten (wie bereits im Abschnitt 4 beschrieben) erzeugt eine äußerst wünschenswerte Abstimmung zwischen den beteiligten Forschungsinstituten und Industriepartnern. Es stellt sicher, dass die Industrieperspektive und Industrieinteressen in den Verbundprojekten adäquat berücksichtigt werden.

\subsection{Thematische Schwerpunkte}

Die geförderten Themen in den zwölf Bekanntmachungen der Jahre 1999 bis 2004 spiegeln die große inhaltliche Breite der im Rahmenkonzept angesprochenen Herausforderungen in der deutschen Produktionswirtschaft wider. Trotzdem wird unmittelbar deutlich, dass in den Bekanntmachungen nicht die gesamte Palette der formulierten Herausforderungen im Rahmenkonzept „Forschung für die Produktion von morgen“ in gleicher Intensität verfolgt werden konnte. Bei der Programmumsetzung wurden Schwerpunkte gesetzt. Dies erscheint uns angesichts des umfassenden Anspruchs des Programms und der beschränkten zur Verfügung stehenden Fördermittel auch angemessen und nachvollziehbar.

Betrachten wir die Themen in den zwölf Bekanntmachungen über die Programmlaufzeit, so fällt auf, dass sich zu Beginn des Programms die Bekanntmachungen noch eng in den im Rahmenkonzept beschriebenen Themenfelder widerspiegeln. Bei den späteren Bekanntmachungen ist der Bezug zu den im Rahmenkonzept angeführten Themen nicht mehr unmittelbar offensichtlich. Über die Zeit hat hier das Prinzip „offenes und lernendes Programm“ seinen Niederschlag gefunden und die Förderschwerpunkte und Themen wurden neuen Prioritäten angepasst.

Eine kontinuierliche Anpassung des Rahmenkonzepts im Lichte neuer Entwicklungen ist grundsätzlich zu begrüßen. Gleichzeitig sollte jedoch sichergestellt sein, das dieser Prozess der laufenden Adaptierung bewusst und dokumentiert erfolgt. Auch die Umsetzung eines lernenden Programms darf sich nicht beliebig weit vom ursprünglichen Anspruch des Rahmenkonzepts entfernen. Gerade ein lernendes Programm braucht feste Koordinaten, um begründet und schlüssig eine Entscheidung darüber treffen zu können, was im Lichte dieser festen Koordinaten gefördert wird und was nicht bzw. was noch Programmgegenstand sein kann und was nicht.

Wir würden diesen Punkt an dieser Stelle nicht so betonen, hätten wir nicht den Eindruck, dass über die zu evaluierende Programmperiode der im Rahmenkonzept prominent verankerte systemische Anspruch des Programms über die Jahre ein wenig in den Hintergrund geraden ist. Wie wir bereits im Abschnitt 5.2 gezeigt haben, hat der Anteil organisations- und strategieorientierter Themen in den Bekanntmachungen und damit auch der geförderten Verbundprojekte stetig abgenommen. Insbesondere das Handlungsfeld 4: Der Mensch und das wandlungsfähige Unternehmen tritt im Laufe der Umsetzung des Rahmenkonzepts gemessen an den geförderten Vorhaben und den zur Verfügung gestellten Mitteln etwas in den Hintergrund. 
Trotzdem konnten in einigen geförderten Verbundprojekten explizite Verbindungen zur Aus- und Weiterbildungsthemen hergestellt werden. Auch ist uns bewusst, dass durch Verbundforschung zwischen Hochschulinstitute und Industrie auch ein wesentlicher Beitrag zu Rückkopplung von Forschungsergebnissen in die universitäre Lehre geleistet wird. Daneben darf der indirekte volkswirtschaftliche Nutzen durch die Ausbildung von Doktoranden und Diplomanten im Rahmen von anwendungsrelevanten Forschungsthemen nicht unterschätzt werden. Auch ist bei vielen technischen Projekten (wie die Fallstudie zum Verbundprojekt ChopArc exemplarisch zeigt) das wichtigste Ergebnis ohnehin "talent not technology“. Trotzdem erscheint uns eine stärkere Berücksichtung dieses Themas in Zukunft durchaus wünschenswert zu sein. Ähnliches gilt für das Handlungsfeld 3: Neue Formen der Zusammenarbeit produzierender Unternehmen.

In vergleichsweise geringem Umfang wurden in der Periode 1999 bis 2004 auch dezidiert ganzheitliche und nachhaltige Lösungen für Produktionssysteme in Bekanntmachungen direkt adressiert. Zwar ergeben sich bei Themen wie Servicing, Dienstleistungen und Life-Cycle Themen, die in einigen der Verbundprojekten aufgegriffen wurden, unmittelbar Anknüpfungspunkte zu ganzheitlichen und nachhaltigen Lösungen. Trotzdem sollte geprüft werden, ob das Ausmaß der bisherigen Berücksichtung dieses Themas strategisch gewünscht wird.

Wenn also die Beschreibung der grundlegenden Veränderungen und der zentralen Herausforderungen an die Produktion im 21. Jahrhundert, so wie wir sie im Rahmenkonzept beschrieben finden, weiterhin gültig ist - und wir haben allen Grund dies anzunehmen - dann sollte bei der weiteren Umsetzung des Rahmenkonzepts einer angemessenen Berücksichtung aller Handlungsfelder - und insbesondere auch von handlungsfeldübergreifenden Themen - ausreichend Augenmerk geschenkt werden.

\subsection{Beurteilung der Programmadministration durch die Teilnehmer}

Die Arbeit des Projektträgers wird von den teilnehmenden Betrieben und Forschungsinstituten insgesamt als ausgesprochen positiv beurteilt. Sowohl die qualitativen Interviews als auch die Ergebnisse der Teilnehmerbefragung zeigen hier eindeutig in die selbe Richtung.

Der Anteil an positiven Bewertungen in der Teilnehmerbefragung bewegt sich bei den meisten abgefragten Aspekten des Programmmanagements zwischen 85\% und 95\%. Institutspartner beurteilen die Arbeit des Projektträgers etwas positiver als Industriepartner. Besonders hoch ist die Zustimmung der Befragten bei denen Aspekten, die unmittelbar die Betreuungsarbeit des Projektträgers berühren: jeweils mehr als 95\% der Antwortenden stimmten den Aussagen zu bzw. eher zu, dass der die Betreuung des Projektträgers in der Antragsphase bzw. im Verbundprojekt hilfreich war, und dass der Projektträger flexibel auf notwendige Änderungen im Projekt reagiert.

Wahrnehmbare Kritik - und hier interessanter weise stärker von Seite der Institute wird nur bei zwei der abgefragten Aussagen zur Antrags- und Projektabwicklung artikuliert: Insgesamt 38\% der Befragten konnten der Aussage, Die Kriterien zur Beurteilung der Projektskizzen sind transparent' nicht bzw. eher nicht zustimmen. Für ein Drittel der Befragten ist auch die Entscheidung über die Projektskizze nicht 
bzw. eher nicht nachvollziehbar. Damit werden die Ergebnisse der qualitativen Interviews bestätigt, in denen wiederholt Zweifel an der Transparenz des Auswahlverfahrens von Skizzen geäußert wurden.

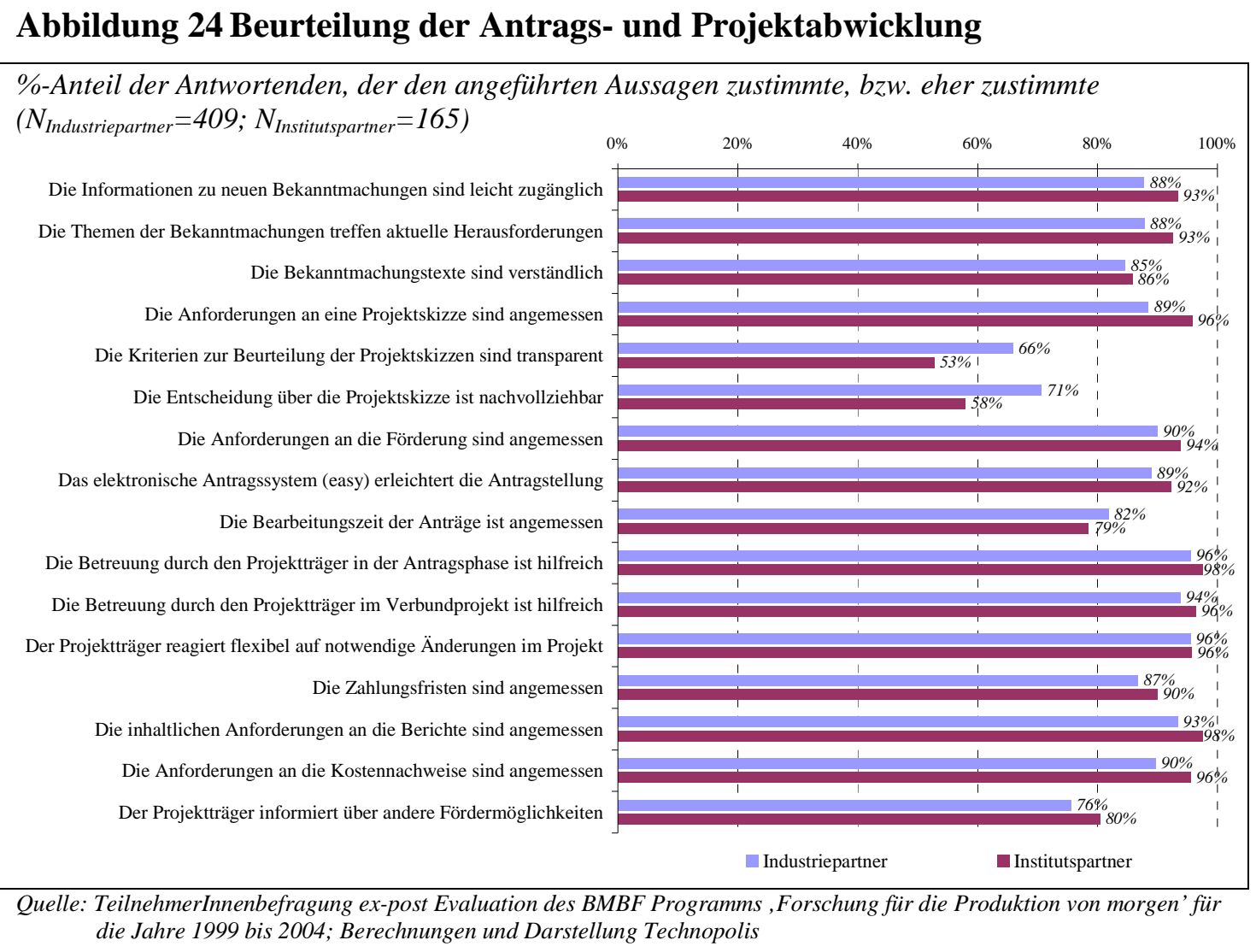

Insgesamt betrachtet weisen die Ergebnisse der Teilnehmerbefragung und aus den Interviews auf einen Projektträger hin, der bei den Programmbeteiligten ein hohes Ansehen genießt und dem eine hohe Kompetenz und Professionalität bei der Abwicklung der Projekte zugesprochen wird. Die Ergebnisse stellen - auch im Vergleich zur Beurteilung anderer Projektträger und Förderagenturen, für die wir in der Vergangenheit ähnliche Aussagelisten abgefragt haben - dem Kerngeschäft des Projektträgers ein sehr gutes Zeugnis aus.

\subsection{Transfermaßnahmen und Öffentlichkeitsarbeit}

Auf den Aspekt Verwertbarkeit der Projektergebnisse für Dritte aus Sicht der Programmteilnehmer sind wir bereits im Abschnitt 6.5 eingegangen. Es ist uns wichtig nochmals festzuhalten, dass das Rahmenkonzept „Forschung für die Produktion von morgen" kein Technologietransferprogramm ist, sondern ein ambitioniertes FuE-Programm, mit dem in erster Linie industriell relevante Spitzenforschung für die Produktion gefördert werden soll. Ein gewisser innerer Widerspruch im Rahmenkonzept ergibt sich allerdings aus dem Umstand, dass als Ziel des Programms die breite Anwendung von Forschungsergebnissen in kleinen und mittleren Unternehmen, besonders in den neuen Ländern, unterstützt werden soll. Es ist aber evident, dass dieses Ziel mit dem zentralen Instrument der Verbundforschung nicht erreicht werden kann. Trotzdem haben wir den Eindruck gewonnen, dass der Projektträger das Thema Transfer bei der Umsetzung des Programms sehr ernst nimmt und im Rahmen seiner bestehenden Möglichkeiten 
versucht, die erzielten Ergebnisse im Programm auch dem ungeförderten zugänglich zu machen.

\section{Industriearbeitskreise}

Das wichtigste Instrument des Projektträgers für den Maßnahmentransfer im Rahmenkonzept ist es, in den Verbundprojekten darauf zu achten, dass industrielle Anwender und Transferpartner ausreichend im Projektdesign berücksichtigt werden. Als darüber hinaus gehendes Instrument für den Ergebnistransfer regt der Projektträger die Projektpartner an, parallel zur Durchführung von Verbundprojekten Industriearbeitskreise (IAK) einzurichten. Die Liste der eingerichteten Industriearbeitskreise ist beeindruckend (aber insgesamt etwas unübersichtlich). Auch der Umstand, dass einige der Industriearbeitskreise noch Jahre nach Abschluss der Verbundprojekte aktiv sind ist, ein Indiz für die Akzeptanz dieses Instruments des Ergebnistransfers. Eine mögliche Maßnahme zur Straffung und Stärkung der IAKLandschaft wäre, dass der Projektträger verstärkt versucht, vermehrt themenfeldorientierte Industriearbeitskreise zu initiieren, die den Bogen über mehrere Verbundprojekte bzw. Anwendungsfelder spannen und so möglicher weise für eine größere Gruppe von ungeförderten Unternehmen Anknüpfungspunkte für eine Mitarbeit und einen gemeinsamen Austausch bieten.

\section{Karlsruher Arbeitsgespräche Produktionsforschung}

Ein Instrument zur Verbreitung der Ergebnisse des Rahmenkonzepts sind auch die Karlsruher Arbeitsgespräche Produktionsforschung (KAG). Ein Arbeitspaket in dieser Evaluation beschäftigte sich detailliert mit der Frage inwieweit durch die KAG Impulse für die Entwicklung von neuen thematischen Schwerpunkten des Rahmenkonzepts gegeben werden. Wir gingen auch der Frage nach, ob die KAG eine Plattform für Wissensaustausch und Anregungen zu unternehmerischen Innovationen bieten. Wir suchten nach Anhaltspunkte für Beiträge zur Verbreitung und zum Transfer der Forschungsergebnisse. Schließlich prüften wir, in welchem Ausmaß mit den Karlsruher Arbeitsgesprächen auch KMU (aus den neuen Bundesländern) erreicht werden konnten.

Die Ergebnisse zeigen, dass es dem Projektträger mit den KAG immer wieder gelingt neue Themen aufzugreifen, die später Gegenstand von Bekanntmachungen werden. Der Anteil von TeilnehmerInnen aus der Industrie und als Vortragende liegt bei zwischen $50 \%$ und $60 \%$. Dies lässt eine angemessene Berücksichtigung praxisorientierter Ergebnisse und Ansätze erwarten. Etwa 60\% der industriellen TeilnehmerInnen kommt aus kleinen und mittleren Unternehmen. Die (leider spärlichen) Rückmeldungen von TeilnehmerInnen zu den Arbeitsgesprächen lassen vermuten, dass die KAG als ein wirksames Forum für Kontaktanknüpfungen wahrgenommen werden. Ebenso wird der Nutzen der Veranstaltung für die tägliche und künftige Arbeit von den Betrieben als überwiegend gut bewertet. Die Auswertungen der KAG Teilnehmerlisten geben Hinweise darauf, dass die Veranstaltung auch nicht geförderte Unternehmen erreicht wird: Mehr als ein Drittel der teilnehmenden Industriebetriebe werden nicht als Projektpartner im Rahmenkonzept „Forschung für die Produktion von morgen" gefördert.

Um mehr Beteiligung an den KAG von Unternehmen aus dem nördlichen und östlichen Teilen Deutschlands zu stimulieren, könnte daran gedacht werden, 
Dresdner Arbeitsgespräche alternierend mit bzw. zusätzlich zu den Karlsruher Arbeitsgesprächen zu veranstalten.

\section{Darstellung des Rahmenkonzepts im Internet}

Ein Potenzial für eine verbesserte Verbreitung der Programmergebnisse sehen wir noch im Bereich der Öffentlichkeitsarbeit. Aus unseren Gesprächen und den Auswertungen der Schlussberichte wissen wir, dass auch viele Projektteilnehmer den Wunsch nach mehr Öffentlichkeitsarbeit über das Programm und die Ergebnisse der geförderten Projekte haben. Unter den Anregungen, die sich beispielsweise in den Schlussberichten finden, sind etwa jene nach einem regelmäßigen Newsletter, nach einer verstärkten Präsenz des Programms im Internet mit leicht zugänglichen Informationen über die geförderten Vorhaben, nach mehr Information über geplante und laufende Aktivitäten und Förderschwerpunkte, nach der Einrichtung von Erfahrungskreisen sowie generell nach Förderung von mehr Austausch zwischen verschiedenen, thematisch verwandten Verbundprojekten.

Mit der Neugestaltung der Internet Plattform www.produktionsforschung.de ist sicherlich schon ein wichtiger Schritt gesetzt worden. Trotzdem glauben wir, dass durchaus noch Potenzial für eine intensivere Vermarktung des Rahmenkonzepts „Forschung für die Produktion von morgen“ und seiner Ergebnisse vorhanden ist. Die bisher am Internet verfügbaren Projektbeschreibungen sowie die angegebenen Kontaktdetails des Koordinators / der Koordinatorin, des / der zuständigen Betreuers / Betreuerin beim Projektträger und gegebenenfalls der Verweis auf eine Webseite des Verbundprojekts erscheinen zu technisch und zu wenig nutzerfreundlich um Interessierten einen leichten Zugang zu den geförderten Projekten zu ermöglichen. Ein möglicher erster Schritt zu einer besseren Zugänglichkeit der Projektergebnisse wäre, attraktiv aufgearbeitete, ergebnisorientierte Kurzberichte über die geförderten Vorhaben zum direkten Herunterladen von der Internetseite des Rahmenkonzepts anzubieten, in der die Projektpartner insbesondere auch auf das Marktpotenzial bzw. die Verwertung der Ergebnisse eingehen sollten. 


\section{$8 \quad$ Programmstrategie: Anregungen des Experten Panels}

Zentrales Ziel dieser Evaluation war es, die Wirkungen der Förderung im Rahmenkonzept „Forschung für die Produktion von morgen“ zu erheben, um damit die Frage beantworten zu können, ob das Rahmenkonzept bisher seine gesetzten Ziele erreichen konnte. In den vorangegangen Abschnitten haben wir die empirische Grundlage präsentiert, die eine Beantwortung dieser Frage erlaubt.

Die Auseinandersetzung mit der Programmstrategie selbst ist also nicht unmittelbar Gegenstand dieser Evaluation. Trotzdem stellen sich bei jeder Evaluation natürlich unmittelbar Fragen nach der Angemessenheit staatlicher Intervention und nach der Relevanz und Kohärenz der gesetzten Maßnahmen. Gerade bei einem offenen und lernenden Programm wird die Beschäftigung mit diesen Themen ohnehin fester Bestandteil zur kontinuierlichen Weiterentwicklung des Rahmenkonzepts sein. Im Rahmen dieser Evaluation hat sich eine Gruppe von sieben internationalen Experten aus den Gebieten der Innovationsforschung, der Produktionsforschung und der FuEFörderung mit der Strategie und der Umsetzung des Rahmenkonzepts „Forschung für die Produktion von morgen" aus internationaler Perspektive auseinander gesetzt und ihre Beobachtungen und Anregungen zur Weiterentwicklung des Rahmenkonzepts in einem eigenen Panel Bericht zusammengefasst (siehe dazu den Bericht zu Arbeitspaket 9: Experten Panel).

Die zentralen Kommentare des Experten Panels zur Rahmenkonzept möchten wir in diesem Abschnitt zusammenfassend darstellen. Der Panel Bericht kann unserer Meinung nach auch einen Beitrag leisten, die bereits laufenden Aktivitäten des BMBF und des Projektträgers zur Neuorientierung des Rahmenkonzepts „Forschung für die Produktion von morgen" durch das Einbringen einer Außensicht auf das Programm zu unterstützen.

\section{Allgemeiner Programmansatz}

Insgesamt wirkt das BMBF Rahmenkonzept nach Einschätzung des Panels als ein zeitgemäßes und gut durchdachtes Programm, das auf einem klaren und an der Zukunft orientierten Bild von Produktion im 21. Jahrhundert aufbaut: gekennzeichnet vom Bedarf nach einer globalen Positionierung und einem strategischen Verständnis der zugrunde liegenden Veränderungen. Das Leitbild bzw. eine Vision für die Produktion von morgen in Deutschland könnte aber den Mehrwert und den Fokus des Programms noch deutlicher vermitteln. Dazu könnte es möglicher weise stärker als bisher nötig sein, explizit strategische Schwerpunkte hinsichtlich bestimmter Branchen oder Zielmärkte zu setzen.

Nach Ansicht des Panels verfolgt das Rahmenkonzept einen systemischen Ansatz und versucht, auf programmatischer Ebene den Bogen von der Produktentwicklung über die Fertigung bis hin zu Produkt begleitenden Dienstleistungen, Servicing und der Aus- und Weiterbildung zu spannen. Trotzdem wirkt das Programm zu sehr technology push orientiert und eine bessere Balance von Technologieentwicklung mit der Nachfrageseite (d.h. bezüglich Märkte, Anwender und Kunden) wäre wünschenswert. Die Ergebnisse der Innovationsforschung zeigen deutlich, dass für erfolgreiche Innovationen die frühe Einbeziehung von Anwendern oft entscheidend 
ist. Die Stärkung von Innovationskompetenz in Produktionsnetzwerken ist langfristig effektiver als eng definierte Technologieentwicklung.

\section{Weiterentwicklung des Rahmenkonzepts}

Die Schwerpunkte des Programms sind nach Ansicht der internationalen Experten richtig gesetzt, es bräuchte aber häufig eine spezifischere Aus- und Neuformulierung von bestehenden Herausforderungen und Förderschwerpunkten bzw. eine laufende Anpassung des Rahmenkonzepts. Damit könnte dem Anspruch des Rahmenkonzepts, ein ,lernendes Programm' zu sein, noch besser entsprochen werden. Durch das Erstellen und regelmäßige Überarbeiten einer Art Roadmap für das Programm und internationales Benchmarking könnten die Schwerpunkte laufend aktuell gehalten werden. Dadurch könnte auch eine konsistentere Abwicklung der vordringlichen Forschungsthemen in einem systemischen Zusammenhang erreicht werden (vom Produkt über die Fertigungssysteme bis hin zu den dazu notwendigen Komponenten und Werkzeugen).

\section{Internationale Abstimmung}

Das Panel regt auch eine intensivere Abstimmung des deutschen Programms mit den laufenden Aktivitäten im Rahmen von Manufuture an. ,Forschung für die Produktion von morgen' könnte verstärkt als öffentlich finanzierter Teil zur Umsetzung der nationalen Manufuture Strategie in Deutschland begriffen werden. Eine Strategie zur Vernetzung mit internationalen Aktivitäten sowie mit Programmen in anderen Ländern könnte der Gefahr einer zu starken Binnenorientierung des Programms entgegenwirken.

\section{Horizontalisierung}

Die Integration der technologischen, organisatorischen und strategischen Dimension von Innovation in der Produktion sollte nach Ansicht des Panels sowohl im Rahmenkonzept als auch auf Ebene der Bekanntmachungen und der geförderten Projekte stärker zum Tragen kommen. Bereits in der Programmkonzeption sollte eine verstärkte Koordination mit anderen Referaten und relevanten BMBF Programmen erfolgen. Mehr gemeinsame, horizontal orientierte Bekanntmachungen im Programm wären hier wünschenswert.

Generell sollte eine stärkere themenfeldübergreifende Implementierung des Programms bzw. eine stärkere Vernetzung der Themenfelder ins Auge gefasst werden. Diese könnte beispielsweise in Form einer einmal im Jahr durchgeführten offenen horizontalen Bekanntmachung gefördert werden, in der besonders innovative Projektvorhaben, die quer zu den Handlungs- und Themenfeldern des Programms liegen, eingereicht werden können.

\section{Radikale Projekte}

Die geförderten Projekte sind nach Ansicht des Panels insgesamt relevant und technologisch anspruchsvoll, häufig aber eher die Fortschreibung von Entwicklungslinien des 20. Jahrhunderts als Wegweiser in das 21. Jahrhundert: Nur vereinzelt werden radikal neue Ansätze verfolgt. Es besteht damit ein gewisses Missverhältnis zwischen dem ambitionierten Programmanspruch und der inhaltlichen Orientierung 
der geförderten Projekte. Nach Ansicht des Panels sollten bei der Weiterführung des Programms risikoreichere, spekulative und radikal neue FuE-Ansätze stärker als bisher Berücksichtigung finden. Hier wäre zu überlegen, ob dazu mehr Schnittstellen zur Grundlagenforschung etabliert werden müssen bzw. ob dazu ein eigener Projekttypus mit besonderem Finanzierungsmodell angemessen wäre. Das Thema Ausbildung sollte verstärkt in allen geförderten Projekten gefordert bzw. von den Konsortien angesprochen werden.

\section{Fokus auf Ergebnisverwertung und Transfer}

Nach Ansicht des Panels könnten im Rahmenkonzept die Verbindungen von der Projektebene zur industriellen Anwendung noch weiter gestärkt werden. Es sollte kein Projekt gefördert werden, für das überprüfbare Projektziele sowie ein aussagekräftiger Umsetzungs- und follow-on Verwertungsplan fehlen. Gerade in der Vorprojektphase kann durch den Projektträger hier unterstützend eingegriffen werden. Auch im Bereich des (ex-post) Projekt Monitoring sieht das Panel noch ungenützte Potenziale. Die Projektpartner sollten vermehrt dazu angehalten werden, in ihren Berichten Aussagen über die wirtschaftliche Verwertung und Verbreitung der Projektergebnisse zu machen, und diese - soweit möglich - auch zu quantifizieren. Die Wirkung der Förderung könnte so besser eingeschätzt und öffentlich kommuniziert werden.

Angedacht werden könnte nach Meinung der internationalen Experten eine stärkere Differenzierung von Diffusionsmechanismen in Abhängigkeit der Aufnahmefähigkeit der Adressaten: Firmen mit einer hohen Aufnahmekapazität für neue Ideen können möglicherweise schon damit erreicht werden, dass man sie auf die Ergebnisse des Programms verstärkt hinweist. Eine zweite Gruppe von Firmen wird bereits spezifische Unterstützung bei der Anpassung und Implementierung von neuen Lösungen brauchen. Hier könnten aktive Technologietransferakteure eine wichtige Rolle spielen. Schließlich bleibt die große Gruppe der Innovationsnachzügler, die vor allem durch Gruppendruck (peer pressure) oder durch Einbeziehung in Lieferanten- bzw. Wertschöpfungsnetzwerke erreicht werden können. Das BMBF könnte in Betracht ziehen, Projekte zu finanzieren, deren ausschließliches Ziel es ist, die Verbreitung von Forschungsergebnissen zu einem bestimmten Thema, bzw. eines Themenfeldes zu unterstützen. Das Ziel wäre insgesamt, im Rahmenkonzept mehr auf Diffusion zu achten als ausschließlich auf die Förderung industrieller Spitzenforschung.

\section{Messbare Programmziele festlegen}

Die Festlegung von überprüfbaren, bzw. messbaren Programmzielen wäre geeignet, nicht nur den technischen Erfolg der Programmförderung zu betonen, sondern auch den wirtschaftlichen Erfolg und damit die Wirksamkeit des Programms für Deutschland transparenter zu machen. Regelmäßigere Evaluierungen des Programms sind notwendig um dem Anspruch, lernendes Programm' zu sein, gerecht werden zu können.

\section{Informationen über die geförderten Projekte verbessern}

Die verfügbaren Informationen über die Ergebnisse der geförderten Projekte auf den Internetseiten des BMBF und des Projektträgers könnte nach Ansicht des Panels weiter verbessert werden (d.h. mehr und bessere Öffentlichkeitsarbeit über das 
Programm und seine Ergebnisse). Die Sichtbarkeit der Projekte und die Verbreitung der Projektergebnisse durch den Projektträger sollte erhöht werden. Nutzerfreundliche Kurzfassungen der Projektendberichte sowie Informationen über die Anwendung und Verbreitung der Projektergebnisse in den Unternehmen sollten über die Internet-Seite des Programms unmittelbar zugänglich bzw. abrufbar sein.

\section{Neue thematische Schwerpunkte}

Auf thematischer Ebene sieht das Panel für die kommende Programmperiode des Rahmenkonzepts ,Forschung für die Produktion von morgen' vor allem folgende vordringliche Forschungsaufgaben: Das Thema kundenindividuelle Massenproduktion wird auch in den kommenden Jahren relevant bleiben, wobei Aspekte wie Produktindividualisierung als auch Lean \& Agile Manufacturing Solutions an Bedeutung gewinnen werden. Das Thema Simulation und die Entwicklung eines Rahmens für Virtual Factory wird ebenfalls auf den Forschungsagenden der kommenden Jahren stehen. Die zentrale Herausforderung hier ist die Entwicklung einer integrierten Simulationsplattform über den gesamten Produkt- bzw. Produktionszyklus hinweg. Das Thema Prozesse wird in Zukunft ebenfalls wieder stärker in den Vordergrund rücken: In der Organisation, Kompatibilität, Optimierung und Robustheit von Prozessketten sind noch beträchtliche Innovationspotenziale zu eröffnen. In Zusammenhang mit der Überwindung bestehender Grenzen in der Automatisierung treten Themen wie Selbstdiagnose und Selbstreparatur von Produktionssystemen ins Zentrum des Interesses.

Neue Formen der Zusammenarbeit von Unternehmen in Produktionsnetzen und neue Formen der Organisation der Produktion im Unternehmen, einschließlich neuer Konzepte der Montage, sollten nach Ansicht des Panels ebenfalls Teil der Forschungsagenda der kommenden Jahre sein. Die Themen Nachhaltigkeit, Arbeitskräfte einschließlich Ausbildung, Arbeitsgestaltung und Arbeitssicherheit in der Produktion sollten im Programm tatsächlich jenen Stellenwert erhalten, wie im Rahmenkonzept zu den Handlungsfeldern ausgeführt wird.

Hinsichtlich technologischer Schwerpunkte, die in das Programm verstärkt integriert werden könnten, kommen nach Meinung der internationalen Experten vor allem die Themen Mikro- und Nanotechnologie (einschließlich bottom-up Prozesse), optische Verfahren in der Produktion sowie neue Entwicklungen in der Materialwissenschaft und Oberflächentechnik in Betracht. Auch das Thema IKT für traditionelle Produktionsprozesse ist nach wie vor nicht ausgereizt. Besonderes Augenmerk sollte bei der Umsetzung thematischer Schwerpunkte auf Systeminnovationen gelegt werden, durch die Innovationssprünge ermöglicht werden. 


\section{Schlussfolgerungen}

In diesem anschließenden Abschnitt fassen wir die wesentlichsten Ergebnisse und Befunde der Evaluation des Programms „Forschung für die Produktion von morgen“ zusammen und schlagen Handlungsempfehlungen für die Weiterführung des Programms vor.

\subsection{Diskussion der Befunde der Evaluation}

\section{War das Rahmenkonzept „Forschung für die Produktion von morgen“ wirksam?}

Aus den in der vorhergehenden Abschnitten präsentierten Analysen und empirischen Ergebnissen kann der Durchführung des BMBF Rahmenkonzepts „Forschung für die Produktion von morgen“ in den Jahren 1999 bis 2004 hinsichtlich der erzielten Wirkungen in den teilnehmenden Unternehmen ein sehr gutes Zeugnis ausgestellt werden. Die Matched-Pair Analyse, die Ergebnisse der Teilnehmerbefragung und die qualitativen Befunde aus der Dokumentenauswertung, den Interviews und den Fallstudien weisen alle eindeutig in die gleiche Richtung: Durch die Projektförderung im Rahmenkonzept „Forschung für die Produktion von morgen“ in den Jahren 1999 bis 2004 konnten in den teilnehmenden Unternehmen signifikant positive wirtschaftliche Effekte ausgelöst werden.

Sowohl die absoluten FuE-Gesamtaufwendungen der geförderten Unternehmen und ihre FuE-Intensitäten, als auch die absolute Anzahl ihrer MitarbeiterInnen im FuEBereich und ihr Anteil an allen Beschäftigten im Unternehmen sind bei den geförderten Unternehmen signifikant höher als bei vergleichbaren nicht geförderten Unternehmen. Das Rahmenkonzept leistet damit einen relevanten Beitrag, FuEKapazitäten in den beteiligten Unternehmen nachhaltig auszubauen. Es lässt sich zeigen, dass es sich dabei nicht um Mitnahmeeffekte der Förderung handelt, sondern es tatsächlich zu einer zusätzlichen Stimulation der eigenen Forschungsintensität der Unternehmen gekommen ist. Dies gilt auch für die spezielle Zielgruppe des Rahmenkonzepts, den kleinen und mittleren Unternehmen (KMU).

Die Wirkungen der Programmförderung im BMBF Rahmenkonzept, Forschung für die Produktion von morgen' zur Verhaltensadditionalität können insgesamt als sehr zufrieden stellend eingeschätzt werden. Es konnte in der Teilnehmerbefragung kein einziger Förderfall identifiziert werden, bei dem nicht zumindest ein teiladditionales Verhalten (Netzwerkadditionalität, Innovationsniveauadditionalität bzw. Beschleunigungsadditionalität) durch die Förderung ausgelöst wurde.

Fast ein Drittel der Industriepartner von abgeschlossenen Vorhaben (32\%) berichtete über erzielte Umsätze mit Produkten (25\%) oder Dienstleistungen (15\%), die auf den Vorhabensergebnissen aufbauen. Mehr als ein Viertel der Industriepartner verzeichnete durch die Ergebnisse der Vorhaben eine Steigerung der Ertragsstärke und jeder siebte Betrieb konnte aufgrund der Projektteilnahme unmittelbar Kosten senken. Jeder achte Industriepartner gab an, Ergebnisse des Verbundprojekts bereits patentiert zu haben. Fast die Hälfte der Industriepartner gab an, dass die Projektergebnisse unmittelbar einen Beitrag zur Sicherung und Schaffung von Arbeitsplätzen in Deutschland geleistet haben. 
Gerade kleine und mittlere Unternehmen (KMU) scheinen bisher vom Programm überdurchschnittlich profitiert zu haben. Im Vergleich zu Großunternehmen setzen sie an die Programmteilnahme auch höhere strategische Erfolgserwartungen. Im Vergleich zu Großbetrieben sind KMU eher in der Lage, die Projektergebnisse erfolgreich für die Verbesserung der organisatorischen Kompetenz für Innovationen im Betrieb zu nutzen und ihre Marktkompetenz zu stärken.

Hinsichtlich der Verwertung der Projektergebnisse zeigt sich eindeutig, dass ein größerer Anteil geförderter Unternehmen im Betrachtungszeitraum 2003 bis 2005 Produktneuheiten oder für den Markt neue Produkte eingeführt hat als dies bei den vergleichbaren nicht geförderten Unternehmen der Fall ist. Die Ergebnisse zeigen aber auch, dass sich dieser höhere Anteil an Produkt- und Marktinnovatoren noch nicht als kommerzialisierbarer Erfolg, gemessen am Umsatzanteil mit diesen Produktinnovationen oder Marktneuheiten, nachweisen lässt. Dies ist allerdings für ein vorwettbewerblich orientiertes Programm auch nicht anders zu erwarten.

Ein ähnliches Bild sehen wir im Bereich der produktbegleitenden Dienstleistungen. Die geförderten Unternehmen, werden durch die Verbundprojekte dazu angeregt, ein breites und komplementäres Angebot von Dienstleistungen rund um ihr Produkt zu konzipieren und ihren Kunden anzubieten. Das Programm leistet demnach auch einen Beitrag dazu, dass sich die Unternehmen von Produktanbietern hin $\mathrm{zu}$ kompletten Problemlösern für ihre Kunden entwickeln und dadurch die Basis für ihre zukünftige Wettbewerbsstärke weiter verbessern.

Auch im Bereich der technischen Prozessinnovationen stimuliert die Förderung über alle beobachteten Unternehmen, insbesondere die Implementierung und erstmalige Nutzung besonders innovativer Prozesstechniken. In den KMU wird darüber hinaus die Implementierung von Produktionstechniken, die bei größeren Unternehmen bereits breiter genutzt werden, stimuliert. Im Bereich organisatorischer Innovationen deuten die Befunde auf einen positiven Effekt der Förderung auf die Einführung innovativer Formen der Unternehmensorganisation, und hier vor allem bei kundenund ablauforientierten Organisationsprinzipien, hin. Diese Ergebnisse bestätigen den Erfolg und die Wirksamkeit des mit dem Programm verfolgten Innovationsansatzes, mit seinem breiten Mix an technisch und nicht-technisch orientierten Dimensionen.

\section{Hat das Rahmenkonzept seine Zielgruppen erreicht?}

Insgesamt wurden mit dem Rahmenkonzept im Evaluationszeitraum 166 Verbundprojekte mit 1.157 geförderten Projektpartnern unterstützt und dafür 264 Mio. EUR Fördermittel aufgewendet. 60\% der geförderten Industriebetriebe sind kleine und mittlere Unternehmen (KMU) mit weniger als 250 Beschäftigten. Von den $63 \%$ der Fördermittel des Rahmenkonzepts, die Industriebetrieben zugute kommen, entfallen $64 \%$ auf KMU. Die realisierte Verteilung kann vor dem Hintergrund der Ziels, kleine und mittlere Unternehmen angemessen zu beteiligen, als gelungen bezeichnet werden.

Die Beteiligungsquote von Betrieben aus den neuen Bundesländern ist mit etwa $10 \%$ etwas geringer als ihr Gesamtanteil am Verarbeitenden Gewerbe (15\%). In diesem Kontext gilt es jedoch zu beachten, dass sich das Rahmenkonzept insbesondere an Betriebe richtet, die eigene FuE betreiben. Verglichen mit dem Anteil der 
internen betrieblichen FuE-Aufwendungen des Verarbeitenden Gewerbes, der auf Betriebe aus den neuen Bundesländern entfällt (4,2 \%), kann nicht auf eine unterdurchschnittliche Beteiligung ostdeutscher Betriebe geschlossen werden.

Mit über einem Viertel stellt der Maschinenbau die meisten der in den Verbundvorhaben beteiligten Betriebe, wobei insbesondere Hersteller von Werkzeugmaschinen im Vergleich zur Betriebsanzahl im Verarbeitenden Gewerbe überproportional vertreten sind. An zweiter und dritter Stelle der beteiligten Branchen folgen mit knapp 17 \% die Hersteller von Geräten der Medizin-, Mess-, Steuerungs-, Regelungstechnik und Optik, von denen insbesondere die Messgerätehersteller überdurchschnittlich partizipieren sowie der Fahrzeugbau, der mit knapp $9 \%$ der beteiligten Betriebe im Vergleich zur Grundgesamtheit ebenfalls überrepräsentiert ist. Mit $18 \%$ überraschend hoch ist die Quote der beteiligten Betriebe aus wissensintensiven produktionsnahen Dienstleistungsbereichen (Datenverarbeitung und Datenbanken, Forschung und Entwicklung, Dienstleistungen überwiegend für Unternehmen. Dagegen sind Betriebe der Prozessindustrie (insbesondere der Chemischen Industrie) sowie Betriebe aus so genannten Low-Tech-Sektoren (Textil, Bekleidung, Leder, Holz, Nahrungsmittel, etc.) signifikant unterdurchschnittlich in den Verbundvorhaben des Rahmenkonzepts vertreten. Damit setzt das Rahmenprogramm schwerpunktmäßig auf die Förderung neuer Lösungen für Stückgut fertigende Betriebe aus dem Bereich der höherwertigen Technologie, die in Deutschland traditionell stark ist und wesentlich zur positiven Handelsbilanz der deutschen Wirtschaft beiträgt.

Industrielle Anwender sind in $46 \%$ der Verbundvorhaben als geförderte Partner integriert. Bezieht man Industriebetriebe mit der Rolle des Entwicklers für die eigene Anwendung mit ein, so kann davon ausgegangen werden, dass in $72 \%$ der Verbundvorhaben zumindest ein Industriebetrieb beteiligt ist, der die Nachfrage- bzw. Anwenderseite vertritt. Auf der anderen Seite stellt sich die Frage, ob ein $9 \%$-Anteil „echter“ Pilotanwender an den bewilligten Fördermitteln bzw. ein geschätzter „Gesamt-Anwenderanteil“" von 10 bis $15 \%$ der Förderung ausreicht, um den technischen und wirtschaftlichen Marktbezug der entwickelten Lösungen sicherzustellen. In diesem Kontext wäre auch zu untersuchen, ob assoziierte Pilotanwender in ungeförderten Arbeitskreisen eine wirksame Alternative zur geförderten Einbindung sein können.

Geförderte Transferpartner finden sich in etwa $6 \%$ der Verbundvorhaben, in geschätzten weiteren $6 \%$ wirken assoziierte oder Transferpartner im Unterauftrag mit. Diese vergleichsweise geringe Integrationsquote ist vor dem Hintergrund des Ziels des Rahmenkonzepts, die breite Anwendung der entwickelten Lösung insbesondere in KMU sicher zu stellen, vertieft zu beleuchten. Ob attraktive Produkte und Lösungen, die durch die beteiligten Industriebetriebe eigeninitiativ vermarktet werden, immer ,,alleine ihren Markt finden“, kann bezweifelt werden. Inwieweit also ein wirksamer Transfer alleine auf diesem Weg oder über Forschungsinstitute, die verallgemeinerbare Lösungen in die Öffentlichkeit tragen, aber nicht auf diese Aufgabe spezialisiert sind, sichergestellt werden kann, sollte überprüft werden.

Die überwiegende Mehrzahl der beteiligten Forschungsinstitute stammt aus den Fachbereichen Maschinenbau und Verfahrenstechnik (insgesamt 60 \%). Auf den 
weiteren Rängen folgen Institute der Elektrotechnik (12\%), der Wirtschaftswissenschaften $(10 \%)$, der Informatik (6\%) und der Sozialwissenschaften (4\%). Spezifische technologische Kompetenzen jenseits des traditionellen Maschinenbaus werden insbesondere aus den Bereichen Werkstofftechnologien, Lasertechnik, Mikrotechnik bzw. -elektronik sowie mit Abstrichen Oberflächen- oder Beschichtungstechnik mit in die Verbundvorhaben integriert.

\section{Wurde das Rahmenkonzept thematisch angemessen umgesetzt?}

In den zwölf Bekanntmachungen im Evaluationszeitraum 1999 bis 2004 wurden 22 spezifische Themenfelder adressiert. Der Schwerpunkt lag dabei bei den technisch orientierten Themen des Handlungsfelds 2: Technologien und Produktionsausrüstungen. Mehr als die Hälfte der geförderten Verbundprojekte (55\%) und mehr als zwei Drittel der vergebenen Fördermittel entfallen auf dieses Handlungsfeld. Insgesamt $18 \%$ der Fördermittel wurden für die 38 Verbundprojekte (23\%) des Handlungsfelds 1: Marktorientierung und strategische Produktplanung gewidmet. 8\% der Fördermittel entfallen auf die 15 Verbundvorhaben des Handlungsfelds 3: Neue Formen der Zusammenarbeit produzierender Unternehmen und nur etwa 5\% der Förderungen im Rahmenkonzept wurden für Vorhaben des Handlungsfelds 4: Der Mensch und das wandlungsfähige Unternehmen eingesetzt. Vor dem Hintergrund des systemischen Anspruches des Rahmenkonzepts „Forschung für die Produktion von morgen" und seiner spezifischen Zielsetzungen wäre aus Sicht des Evaluationsteams eine stärkere Berücksichtung der Themenfelder 3 und 4 in der Programmperiode 1999 bis 2004 dem Rahmenkonzept angemessen gewesen.

\section{Konnten die Ziele des Rahmenkonzepts erreicht werden?}

Hinsichtlich der allgemeinen Ziele des Rahmenkonzepts kann aufgrund unserer Analysen jedenfalls davon ausgegangen werden, dass mit der Förderung der Verbundprojekte ein bedeutender, signifikanter Beitrag zur Stärkung der Innovations- und Wettbewerbsfähigkeit der teilnehmenden Unternehmen, besonders auch der KMU geleistet werden konnte. Die erhobenen Wirkungen des Programms für die Stimulierung von Innovationen in den beteiligten Betrieben belegen dies. Insofern kann begründet angenommen werden, dass mit der bisherigen Programmdurchführung tatsächlich ein relevanter Beitrag geleistet werden konnte, die wirtschaftlichen Ziele des Programms (das sind die internationalen Wettbewerbsbedingungen deutscher produzierender Unternehmen zu verbessern sowie durch die Programmförderung Arbeitsplätze in Deutschland zukunftssicher machen und neue schaffen), tatsächlich zu erreichen. Der Vergleich mit den nicht geförderten Unternehmen im Rahmen der Matched-Pair Analyse zeigt, dass die erzielten Wirkungen in den beteiligten Betrieben ursächlich auf die Programmteilnahme zurückzuführen sind.

Hinsichtlich der im Rahmenkonzept angeführten spezifischen Ziele können wir feststellen, dass das erste genannte Ziel des Rahmenkonzepts, nämlich „,die Forschung und Entwicklung auf dem Gebiet der industriellen Produktion zu stärken, wobei neue Entwicklungen auf Gebieten wie Informations- und Kommunikationstechnik, Oberflächen- und Schichttechnologien, Lasertechnik, neue Werkstoffe genutzt werden“, mit Blick auf die oben bereits geschilderte Einbeziehung spezifischer technologischer Kompetenzen von Forschungsinstituten als jedenfalls erreicht angenommen werden kann. Gleichzeitig möchten wir festzuhalten, dass der erste Teil des Ziels mit dem Instrument der Verbundförderung kaum zu verfehlen 
war. Da keine inferioren FuE Projekte bzw. Projekte außerhalb von Produktion gefördert wurden, musste das Rahmenkonzept FuE auf dem Gebiet der industriellen Produktion stärken.

Zum zweiten spezifischen Ziel des Rahmenkonzepts, ,ganzheitliche und nachhaltige - d.h. ökologische, soziale und ökonomische - Lösungen für Produktionssysteme zu erforschen und dazu die Zusammenarbeit zwischen Industrie und Forschungsinstituten sowie zwischen verschiedenen Wissenschaftsdisziplinen zu vertiefen“ können wir feststellen, dass das Programm sowohl die nachhaltige Zusammenarbeit zwischen Industrie und Forschungseinrichtungen stimuliert hat, als auch verschiedene Wissenschaftsdisziplinen in den geförderten Verbundprojekten im relevantem Ausmaß zusammenarbeiteten. Der ganzheitliche Ansatz spiegelt sich vor allem in der problemorientierten Herangehensweise bei der Formulierung der Bekanntmachungen wider. Allerdings hätte unserer Meinung nach in den Jahren 1999 bis 2004 mehr zur Erforschung (ökologisch) nachhaltiger Lösungen im Sinne des Rahmenkonzepts unternommen werden können.

Im Hinblick auf die Zielsetzung des Rahmenkonzepts, „, die breite Anwendung von Forschungsergebnissen in kleinen und mittleren Unternehmen zu unterstützen und deren Zusammenarbeit in Unternehmensnetzwerken zu erhöhen, zeigen die empirischen Ergebnisse der Teilnehmerbefragung tatsächlich, dass KMU besonders von der Teilnahme am Programm profitieren konnten: Die Fähigkeit von KMU zur Zusammenarbeit in Unternehmensnetzen konnte mit dem Programm erfolgreich stimuliert werden. Allerdings ist die Unterstützung der breiten Anwendung von Forschungsergebnissen in KMU mit dem Instrument der Verbundförderung nicht umfassend zu erreichen. Das Rahmenkonzept ist kein Transferprogramm - und sollte dies auch nicht sein. Von einem FuE-Förderprogramm, dass in mehr als fünf Jahren insgesamt ca. 0,7\% der Betriebe des Verarbeitenden Gewerbes in Deutschland erreicht hat und dafür jährlich ca. $0,08 \%$ der vom Verarbeitenden Gewerbe getätigten FuE Ausgaben zur Verfügung stellte, eine unmittelbare Transferwirkung zu erwarten, wäre unrealistisch. Wir schlagen daher vor, das Wort „breit“ aus der Zielformulierung zu entfernen.

Anstöße zur Anpassung der beruflichen Aus- und Weiterbildung wurden im Rahmen einzelner Verbundprojekte zu Personalentwicklungsthemen im Rahmenkonzept adressiert. Allerdings wären aus unserer Sicht mehr und stärkere Impulse in diesem Bereich der Bedeutung von neuen Anforderungen an die Aus- und Weiterbildung in der Produktion möglich gewesen. Wir sind uns allerdings bewusst, dass es nicht Aufgabe eines industriell orientierten Forschungsprogramms sein kann, dringliche Herausforderungen der Bildungspolitik quasi im Alleingang zu lösen.

Zusammenfassend können wir bezüglich der Erreichung der im Rahmenkonzept formulierten Ziele feststellen, dass die Wirkungen der Umsetzung des Programms in der Evaluationsperiode 1999 bis 2004 insgesamt als sehr gut eingeschätzt werden kann. Bezüglich des Transferziels „,breite Anwendung in KMU“ halten wir es für sinnvoll, die Formulierung des Ziels selbst überdenken, wenn auch zusätzliche Anstrengungen im Bereich der Verbreitung der Projektergebnisse in den ungeförderten Bereich zu begrüßen wären. 


\section{Wie ist das Konzept ,offenes und lernendes Programm'zu bewerten?}

Grundsätzlich ist die laufende Weiterentwicklung der Themen und Förderschwerpunkte als Antwort auf neue Herausforderungen im Sinne des Konzepts „lernendes Programm“ zu begrüßen. Allerdings sollten Änderungen und Neuorientierungen dokumentiert und für Dritte nachvollziehbar erfolgen. Die grundlegenden Leitlinien des Rahmenkonzepts müssen auch bei einem lernenden Programm im Auge behalten werden.

\section{Wird die Umsetzung des Rahmenkonzepts effizient administriert?}

Die organisatorischen und administrativen Abläufe von der Bekanntmachung über die Skizzenauswahl bis zur Betreuung der geförderten Projekte sind klar strukturiert und in den internen QS Dokumenten des Projektträgers sehr gut dokumentiert. Unsere Aufenthalte beim Projektträger in Karlsruhe und Dresden haben uns in der Annahme bestärkt, dass das QS System in der Praxis tatsächlich auch gelebt wird.

Die Arbeit des Projektträgers wird von den Programmteilnehmern aus Industrie und Forschung insgesamt ausgesprochen positiv beurteilt. Auch im Vergleich zu uns zur Verfügung stehenden Ergebnissen aus anderen Evaluationen erscheint die Bewertung des Projektträgers durch die Teilnehmer des Programms hervorragend. Als am häufigsten genannte Kritikpunkte im Verfahren der Antrags- und Projektabwicklung wird die Skizzenbewertung und -auswahl thematisiert. Diese lässt nach Angaben der Teilnehmer bezüglich Transparenz der Beurteilungskriterien und Nachvollziehbarkeit der Entscheidung über die Weiterverfolgung der Skizzen ein Verbesserungspotenzial erkennen.

Im Hinblick auf die verfügbaren Informationen über geförderte Verbundprojekte im Internet fällt auf, dass sich diese häufig auf die technischen Aspekte der Vorhaben konzentrieren während die Vermarktungs- und Verwertungsdimension in den Beschreibungen häufig zu kurz kommt. Auch ist im allgemeinen auf der InternetSeite des Projektträgers kein Zugriff auf die Ergebnisberichte von bereits abgeschlossenen Vorhaben möglich.

\subsection{Handlungsempfehlungen}

Bevor wir unsere Empfehlungen im einzelnen präsentieren, möchten wir festhalten, dass wir auf die zentrale Frage in dieser Evaluation, nämlich welchen Beitrag das Programm und hier insbesondere sein zentrales Instrument, die Verbundprojektförderung, zur Stärkung der technologischen und wirtschaftlichen Wettbewerbsfähigkeit des deutschen Produktionssektors, insbesondere der KMU, leisten konnte, antworten können: Es konnte einen beachtlichen und signifikanten Beitrag leisten! Als Ansatzpunkte für die Weiterentwicklung des Rahmenkonzepts im Sinne der übergeordneten Programmzielsetzungen sehen wir folgende Bereiche:

1 Die im Rahmenkonzept formulierten Herausforderungen und Ziele sowie die zur Verfügung stehenden Instrumente sollten im Lichte der Evaluationsergebnisse reflektiert und gegebenenfalls angepasst werden. Dies betrifft beispielsweise den Anspruch, die breite Anwendung von FuE-Ergebnissen in KMU unterstützen zu wollen. Unser Vorschlag ist, das Wort „breite“ zu streichen. Sollte allerdings das Ziel in seiner jetzigen Form beibehalten werden, muss klar sein, dass die bisher 
dem Programm zur Verfügung stehenden Instrumenten nicht geeignet sind, das Ziel umfassend erreichen zu können.

2 Im Hinblick auf den Anspruch ein ,lernendes Programm“ zu sein, sollte das BMBF die Rahmenbedingungen schaffen, um eine dokumentierte kontinuierliche Weiterentwicklung des Rahmenkonzepts bezüglich der Herausforderungen und Schwerpunkte der Förderung zu erlauben. Ein mögliches Instrument dazu wäre eine in regelmäßigen Abständen (z.B. alle zwei Jahre) fortgeschriebene strategische Planung des Rahmenkonzepts und der Erstellung einer mittelfristigen Programm-Roadmap. Dadurch könnte die Kohärenz zwischen Programmanspruch und Umsetzung jedenfalls gewährleistet werden.

3 Gerade ein „lernendes Programm“, das kein vordefiniertes Enddatum hat, braucht ein Monitoring- und Evaluationskonzept, in dem die Datenerfordernisse, beobachtbare und aussagekräftige Indikatoren und die Methoden festgelegt sind, an Hand derer eine Beurteilung möglich wird, ob die Umsetzung des Programms in Richtung der angestrebten Ziele weist. Dazu gehört auch eine regelmäßige expost Evaluation des Programms in etwa fünfjährigen Abständen. Dazu gehört aber auch das Nachzeichnen der Kommerzialisierungserfolge der geförderten Verbundvorhaben ex-post durch den Projektträger, gegebenenfalls mit externer Unterstützung. Eine standardisierte, sehr straffe jährliche Berichtung der Kommerzialisierungs- und Transfererfolge über einen Zeitraum von fünf Jahren nach Abschluss der Verbundprojekte bei den beteiligten Partnern könnte dazu einen Beitrag leisten.

4 Der integrative Programmanspruch, der sich in den vier Handlungsfeldern des Rahmenkonzepts widerspiegelt ist innovativ und nach wie vor stimmig. Allerdings sollte in der kommenden Programmperiode in der Umsetzung des Rahmenkonzepts verstärkt auf eine ausgewogene Berücksichtigung aller Handlungsfelder geachtet werden. Mehr horizontale Bekanntmachungen, also solche die mehrere Handlungsfelder gleichzeitig adressieren, erscheinen im Lichte der Programmzielsetzungen wünschenswert.

5 Besonders innovative Konzepte finden sich häufig an den Schnittstellen von Themen, Handlungsfeldern oder Programmen. Es besteht dabei die Gefahr, dass solche Ideen im leeren Raum zwischen fachlichen und administrativen Zuständigkeiten verloren gehen bzw. entsprechende Signale aus der Industrie und Forschung keinen Resonanzkörper finden. Wir sind uns bewusst, dass eine Horizontalisierung von Bekanntmachungen über verschiedene Programme hinweg schwierig ist, auf Widerstände stoßen kann, und nur dann Aussicht auf Erfolg hat, wenn dadurch tatsächlich dringliche Probleme angesprochen werden. Trotzdem glauben wir, dass das Kooperations- und Abstimmungspotenzial mit anderen Förderschwerpunkten des BMBF noch nicht ausgereizt ist. Programmübergreifende Bekanntmachungen sollten daher häufiger in Betracht gezogen werden.

6 In der Programmperiode 1999 bis 2004 wurden vor allem Stückgut fertigende Betriebe aus höherwertigen Technologiebereichen des deutschen Verarbeitenden Gewerbes gefördert, die traditionell zu den Stärken der deutschen Wirtschaft gehören. Betriebe der Prozessindustrie sowie Betriebe aus Low-Tech-Sektoren 
wurden bislang mit dem Rahmenkonzepts kaum erreicht. Hier ist auch mit Blick auf die Portefeuilles anderer Fachprogramme zu klären, ob diese Schwerpunktsetzung richtig und strategisch gewollt ist.

7 Das Rahmenkonzept „Forschung für die Produktion von morgen“ könnte in Zukunft stärker strategisch dazu genutzt werden, deutsche FuE Politik im Bereich der Produktionsforschung intensiver in multilaterale, europäische oder auch bilaterale Kooperationen einzubringen. Dies könnte längerfristig positive Auswirkungen auf die stärkere Berücksichtigung deutscher FuE Schwerpunkte und Interessen in der internationalen Forschungszusammenarbeit zeigen, beispielsweise bei der Umsetzung von Manufuture.

8 Bei der Auswahl der Verbundprojekte sollten verstärkt hochinnovative Vorhaben, die ambitionierte neue Ansätze im Sinne des integrativen Anspruchs des Rahmenkonzepts verfolgen, besonders berücksichtigt werden.

9 Der Integration von Anwendern und der Erstellung von geeigneten Markt- und Transferstrategien (mit empirisch feststellbaren Zielen) sollte bei der Auswahl der Skizzen und im Vor-Projekt-Verfahren eine besondere Bedeutung zugemessen werden. Als Fördergrundsatz sollte gelten: In jedem geförderten Projekt muss die Nutzer- und Anwenderperspektive integriert sein!

10 Die Umsetzung des Programms wird vom Projektträger ausgesprochen gut administriert. Verbesserungspotenziale im Verfahren sehen wir noch in einer begründeten standardisierten Rückmeldung der Entscheidung über die Skizzen (ähnlich der derzeitigen EU Rahmenprogrammpraxis), um dadurch Lerneffekte zu ermöglichen und insgesamt die Transparenz des Verfahrens zu erhöhen. Um gleiche Voraussetzungen im Auswahlverfahren sicher zu stellen, sollten die weiterverfolgten Skizzen ausschließlich aus dem Kreis der im Auswahlgespräch präsentierten Vorhaben stammen. Die Ergebnisse der Auswahlverfahren sollten auf den Internet-Seiten des Projektträgers veröffentlicht werden.

11 Die auf den Internet Seiten des Projektträgers zum Rahmenkonzept angebotenen Informationen über laufende und abgeschlossene Verbundprojekte erscheinen uns verbesserungswürdig. Die Projektendberichte sollten in Form von ansprechend gestalteten, informativen Broschüren zum direkten Herunterladen zur Verfügung stehen. Die Verbundpartner sollten in die Pflicht genommen werden, neben dem technischen Endbericht, auch einen transferorientierte Aufbereitung der Projektergebnisse zu veröffentlichen.

12 Industriearbeitskreise könnten verstärkt entlang der Themenfelder des Rahmenkonzepts eingerichtet werden (und weniger entlang einzelner Verbundprojekte), um so das Interesse einer breiteren Klientel zu wecken. 


\section{Appendix A Verzeichnis der Arbeitspaketberichte}

Geyer, Anton und Jürgen Streicher: Ex-post Evaluation des Forschungsprogramms „Forschung für die Produktion von morgen“ für die Jahre 1999 bis 2004. Bericht zum Arbeitspaket 1: Auswertung der Dokumente beim Projektträger. Technopolis, Januar 2006. Wien.

Kinkel, Steffen, Spomenka Maloca und Oliver Som: Ex-post Evaluation des Forschungsprogramms „Forschung für die Produktion von morgen“ für die Jahre 1999 bis 2004. Bericht zum Arbeitspaket 2: Klientelanalyse. Fraunhofer Institut für System- und Innovationsforschung, Mai 2006. Karlsruhe.

Kinkel, Steffen, Barbara Breitschopf, Spomenka Maloca und Oliver Som: Ex-post Evaluation des Forschungsprogramms „Forschung für die Produktion von morgen " für die Jahre 1999 bis 2004. Bericht zum Arbeitspaket 3: Produktionstechnische Wirtschaft und Wissenschaft. Fraunhofer Institut für System- und Innovationsforschung, Oktober 2006. Karlsruhe.

Kinkel, Steffen, Bernd Ebersberger und Angela Jäger: Ex-post Evaluation des Forschungsprogramms ,Forschung für die Produktion von morgen “ für die Jahre 1999 bis 2004. Bericht zum Arbeitspaket 4: Ökonometrische Wirkungsanalyse. Analyse der Input- und Output-Additionalitäten der Förderung. Fraunhofer Institut für System- und Innovationsforschung, Oktober 2006. Karlsruhe.

Geyer, Anton und Alexandra Rammer: Ex-post Evaluation des Forschungsprogramms „,Forschung für die Produktion von morgen“ für die Jahre 1999 bis 2004. Bericht zum Arbeitspaket 5: TeilnehmerInnenbefragung. Technopolis, Mai 2006. Wien.

Hafkesbrink, Joachim und Monika Rudeloff: Ex-post Evaluation des Programms „Forschung für die Produktion von morgen“ des BMBF für die Jahre 1999 bis 2004. Thesen und Befunde zu den Programmwirkungen auf Basis der Stakeholder-Interviews. Bericht zum Arbeitspaket 6. ARÖW, Januar 2006. Duisburg.

Hafkesbrink, Joachim, Michael Krause und Markus Schroll: Ex-post Evaluierung des BMBF-Forschungsprogramms „Forschung für die Produktion von morgen“ für die Jahre 1999 bis 2004. Fallstudien bei ausgewählten Verbundprojekten. Bericht zum Arbeitspaket 7. ARÖW, Oktober 2006. Duisburg.

Hafkesbrink, Joachim und Monika Rudeloff: Ex-post Evaluierung des BMBFForschungsprogramms „Forschung für die Produktion von morgen“ für die Jahre 1999 bis 2004. Auswertung der Karlsruher Arbeitsgespräche 2000, 2002 und 2004. Auswertung der Teilnehmerbefragung 2006. Bericht zum Arbeitspaket 8. ARÖW, Oktober 2006. Duisburg.

Bessant, John, Claudio Boër, Harry Boer, Jan Willem Gunnink, Jos Pinte, Reijo Tuokko und Konrad Wegener: Ex-post Evaluation des Forschungsprogramms „Forschung für die Produktion von morgen“ für die Jahre 1999 bis 2004. Arbeitspaket 9: Bericht des Experten Panels. Technopolis, Oktober 2006. Wien. 


\section{Appendix B Leistungsbeschreibung}

(Auszug)

\section{Zielsetzung und Grundlagen der Evaluation}

[...] Ziel der Evaluation des Programms „Forschung für die Produktion von morgen“ der Jahre 1999-2004 ist es, die Wirkungen der Förderung der Produktionsforschung vor dem Hintergrund der gesetzten Ziele allgemein und instrumentenspezifisch darzustellen, zu bewerten sowie damit die Informationen zusammen zu tragen, die den politischen Entscheidungen über das $\mathrm{Ob}$ und Wie einer künftigen Förderung in zukunftsträchtigen Feldern des produktionsrelevanten Bereichs zu Grunde gelegt werden können.

Im Rahmen dieser ex-post Evaluation soll vor allem die technische und wirtschaftliche Entwicklung der Produktionsforschung in Deutschland und die Wirkung der Fördermaßnahme im Sinne einer Standortbestimmung herausgearbeitet werden. Dabei hat die Evaluation zwei Aspekte: Die Charakterisierung und Einschätzung der Status-quo Situation der Produktionsforschung in Deutschland sowie die Auswertung der Erfahrungen mit der bisherigen Förderung, d.h. der Programmstrategie, den eingesetzten Instrumenten und etablierten Vorgehensweisen in Bezug auf die gesetzten Ziele. Dabei ist insbesondere zu überprüfen, welche potenziellen Anwender erreicht und welche nicht erreicht werden konnten, inwieweit die angestrebten Wirkungen tatsächlich erzielt und wo Hemmnisse vorgefunden wurden und wo ggf. neuralgische Punkte liegen, an denen ggf. Verbesserungen sinnvoll ansetzen können.

Es gilt, den ökonomischen und gesellschaftlichen Nutzen, der mit den eingesetzten Ressourcen erzielt wurde, zu beschreiben. Dahinter steht die Frage nach der Effektivität und Effizienz des Programms und der eingesetzten Instrumente. Ein besonderer Augenmerk ist dabei auf die Frage zu legen, inwiefern besonders die spezielle Zielgruppe des Programms, die kleinen und mittleren Unternehmen (KMU), von ihm profitiert haben. Hierbei ist die Art der Programmdurchführung angemessen zu berücksichtigen.

Entscheidend für den Erfolg und die Wirkung der Förderung der Produktionsforschung wird das Zusammenspiel zwischen

- der Fördermaßnahme und der Ausgestaltung des Förderinstrumentariums,

- den exogenen Markt- und Technologietrends sowie

- den Innovationsproblemen der KMU als einer besonderen Zielgruppe

angesehen. Die Untersuchung muss daher bei der ex-post Evaluation die Notwendigkeit einer optimalen Verknüpfung dieser drei Aspekte im Blick behalten.

\section{Evaluierungsbereiche}

Als Schwerpunkt der Ex-Post-Evaluation soll geprüft werden, ob und in welchem Umfang die angestrebten ursprünglichen Ziele des Forschungsprogramms erreicht worden sind (Zielerreichungskontrolle), ob die Fördermaßnahme für die erreichten 
Ziele und Effekte ursächlich war (Wirkungskontrolle) und ob sie sich als wirtschaftlich erwiesen hat (Wirtschaftlichkeitskontrolle). Darüber hinaus soll die Ex-Post-Evaluation Auskunft darüber geben, ob die operative Programmumsetzung und das eingesetzte Instrumentarium den Anforderungen der Zielgruppen entsprachen. Die Ex-Post-Evaluation soll u. a. folgende Bereiche umfassen:

- Fakten und Basisinformationen

- Struktur und Kennziffern der produktionstechnischen Wirtschaft und Wissenschaft, Entwicklung seit 1999

- Aufteilung der Förderung nach Technologiefeldern, Zielgruppen, Regionen, Innovationsgrad

- Wirtschaftliche und technologische Effekte

- Direkte Kommerzialisierungserfolge des Programms - neue bzw. verbesserte Produkte, Prozesse, Dienstleistungen, Verwertung, Hemmnisse

- Einfluss auf Innovationsverhalten und technologische Kompetenz der Unternehmen; Engpässe, Defizite

- Auswirkungen auf Wettbewerbsfähigkeit und Arbeitsplätze

- Nutzen für KMU

- Bedeutung für Ausbildung und Qualifikation

- Operative Programmumsetzung

- ziel- und themenorientiert, Berührungspunkte mit der EU-Förderung

- Antragsbearbeitung, Projektbetreuung, Beratung, Aufwand 